\title{
LA-UR-18-31604
}

Approved for public release; distribution is unlimited.

Title:

Surveillance Report on SAVY 4000 and Hagan Nuclear Material Storage Containers for Fiscal Year 2018

Author(s):

Karns, Tristan

Oka, Jude M.

Reeves, Kirk Patrick

Kaufeld, Kimberly Ann

Smith, Paul Herrick

Stone, Timothy Amos

Narlesky, Joshua Edward

Kelly, Elizabeth J.

Intended for: Report 
Disclaimer:

Los Alamos National Laboratory, an affirmative action/equal opportunity employer, is operated by Triad National Security, LLC for the National Nuclear Security Administration of U.S. Department of Energy under contract 89233218CNA000001. By approving this article, the publisher recognizes that the U.S. Government retains nonexclusive, royalty-free license to publish or reproduce the published form of this contribution, or to allow others to do so, for U.S. Government purposes. Los Alamos National Laboratory requests that the publisher identify this article as work performed under the auspices of the U.S. Department of Energy. Los Alamos National Laboratory strongly supports academic freedom and a researcher's right to publish; as an institution, however, the Laboratory does not endorse the viewpoint of a publication or guarantee its technical correctness. 


\section{Surveillance Report on SAVY 4000 and Hagan Nuclear Material Storage Containers for Fiscal Year 2018}

Tristan M. Karns (NPI-9)

Jude M. Oka (NPI-9)

Kirk Reeves (E-6)

Kimberly Kaufeld (CCS-6)

\author{
Joshua Narlesky (AMPP-4) \\ Timothy A. Stone (NPI-9) \\ Paul H. Smith (SPE-2) \\ Elizabeth Kelly (CCS-6)
}

\section{Executive Summary}

A Surveillance Program is in place to assess how nuclear material storage containers at LANL are aging in-service. This program is guided by the LANL Surveillance Plan [1] which is required by DOE M441.1-1 requirements, and is modified as necessary to ensure that any issues identified during surveillance or laboratory studies are examined in future surveillances, and that any lifetime implications are taken into account. Under the plan, overall container integrity is evaluated (visual inspections and photographs), and helium leakage rate, O-ring durometer and compression set, and filter performance are measured. The containers for surveillance are chosen annually based on the previous surveillance program results and observations. The surveillance plan targets items believed to provide the greatest challenge to the SAVY-4000 container integrity.

Surveillance items for the year consisted of 7 SAVY 4000 storage containers, 8 Hagan containers, and 21 SAVY 4000 transfer containers. The SAVY 4000 surveillance items ranged in age from 2.0 year to 6.6 years and the Hagan containers ranged in age from 6 years to 17 years. As in FY17, the surveillance containers for this year were selected primarily to better understand the extent of corrosion of the stainless steel components of the containers. Accelerated aging studies indicate that the O-ring and filter components of the SAVY-4000 will last at least 40 years under LANL storage conditions, and observations in surveillance are consistent with the conclusions from those studies. However, the observation of corrosion on the inside of SAVY 4000 and Hagan surveillance containers has shifted the emphasis to an ongoing effort to understanding both the nature and the extent of corrosion on the stainless components, primarily the container body. The restriction on handling soluble residues greater than 500 grams continued most of this year, thus limiting the ability to perform surveillance on these items to a single item. The remaining $>500$ gram items that were identified for surveillance in the FY18 plan will be reconsidered for processing in FY19 and beyond.

Of the 7 SAVY 4000 containers surveilled, two had to be introduced into a glove-box due to the possibility of a failed inner bag-out bag. Data presented on these two items was derived from photographs. In addition, of the 8 Hagan containers surveilled this year, 6 of them had to be introduced into a glovebox due to similar considerations, and data presented on these six items is also derived from photographs. The need to introduce many of the items into a glovebox is not surprising given that surveillance items are specifically chosen to be the most challenging to the containment barrier. Unfortunately, introduction into the glovebox limits the ability to perform quantitative tests on the 
container seals, filters, etc. To compensate for this, in the past year a systematic categorization methodology for ranking the degree of corrosion based on photographic evidence was developed and applied to all surveillance containers to date. A high-level summary of the surveillance results for the SAVY and Hagan containers this year is as follows:

- Container Integrity - Visual inspections on 5 SAVY containers and 2 Hagan containers were completed on the containers that were not required to be introduced into a glovebox. A total of 4 of the 7 total SAVY containers surveilled were found to have corrosion (two of which were the SAVYs that were introduced). The corrosion on the two SAVYs that could be fully visually inspected outside of a glovebox was judged to be unlikely to compromise the container integrity; therefore, all 5 SAVY 4000 containers were returned to service after photos were taken for continued annual surveillance (with the same content). All of the 5 SAVY and 2 Hagan containers that underwent visual inspection passed functional checks of the closure system with no other signs of damage. Corrosion was observed on 3 Hagan containers that had been introduced and 1 Hagan of the 2 Hagans that underwent additional evaluations.

- O-rings - O-ring visual inspections, durometer measurements and helium testing were completed on 5 SAVY and 2 Hagan containers. Two SAVY-4000 O-rings were found with small flaws (likely manufacturing defects), but they were not substantial enough to compromise the O-ring seal as determined by helium leak testing. All 5 SAVY containers evaluated passed the helium leak test criteria, O-ring visual inspection criteria, and durometer specifications. A single Hagan container with minimal corrosion failed the helium leak test. The Hagan O-ring for this container was slightly harder than expected, and it is likely that the failure was at the O-ring seal. The other Hagan passed all test criteria. Helium leak testing results also confirmed that the containment system for the 5 SAVYs and 1 Hagan tested were fully intact.

- Filters-No issues were found with the SAVY 4000 or Hagan lid filters tested. The 5 SAVY and 2 Hagan containers met the test criteria for capture and pressure drop indicating no degradation in efficiency and no evidence of filter clogging.

- This report also contains a trending analysis (Section 4.3) of the measurements performed during surveillance testing on SAVY containers (helium leak, O-ring durometer and compression set, and filter performance). Trending analysis of filter performance and O-ring properties showed essentially no change in their performance and properties over the past five-year surveillance period. In addition to this report, the surveillance activities to date are documented in three surveillance reports [2] [3] [4]. The report has also improved by implementing a ranking of the serverity of corrosion, a validation of the bag degredation factor which will be used to target new storage containers for surveillance. 
Key recommendations from the FY17 surveillance report are being actively worked as follows:

- Training on storage container operating procedures have been enhanced to emphasize how to spot corrosion and the necessity to follow through with the appropriate notifications to evaluate any concern.

- A semi-automated system for measuring wall thickness for items identified as having corrosion or to assess potential corroded items in an as-found condition (while still loaded with contents) continues to move forward primarily dependent on availability of students who have been key in developing the system to also have opportunity to be involved in the implementation.

- A new bag out bag material has been determined to be viable to replace the sPVC bag out bag with inherent incompatibility issues $(\mathrm{HCl}$ generation due to $\mathrm{PVC}$ degradation) in the current storage container packaging configuration. The next phase of implementation involves use of the new bag out bag as early as practicable when re-packaging SAVY surveillance containers that are pulled for evaluation in FY19.

New recommendations have been documented in the FY19 version of the Surveillance Plan and are reiterated in Section 6 of this report. 


\section{Table of Contents}

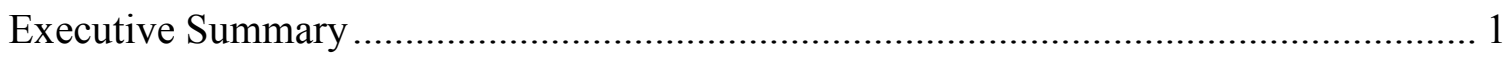

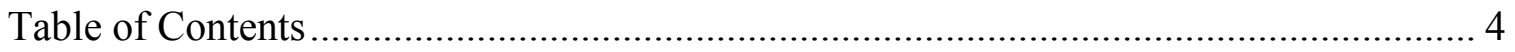

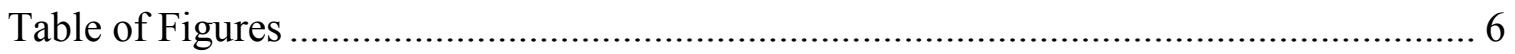

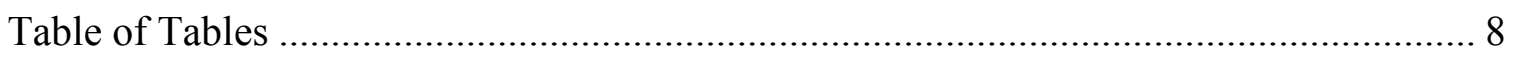

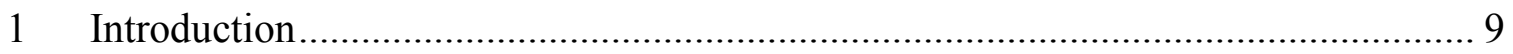

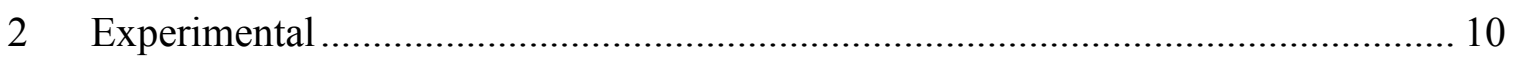

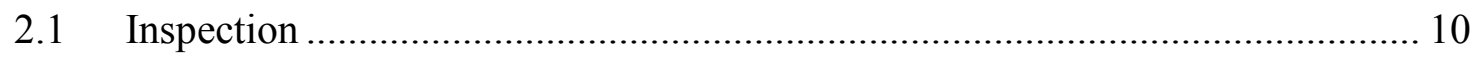

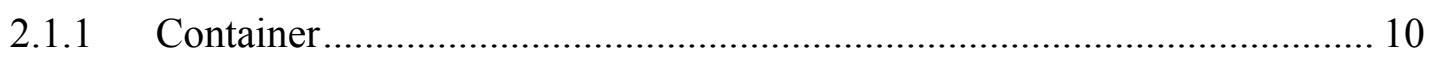

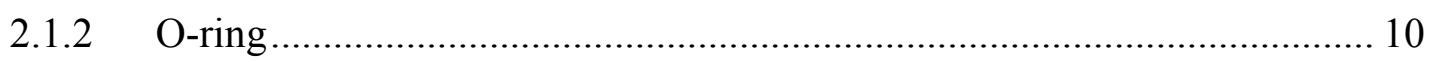

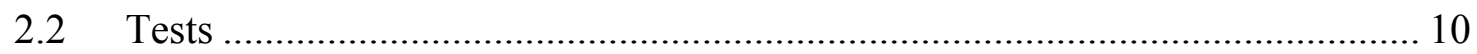

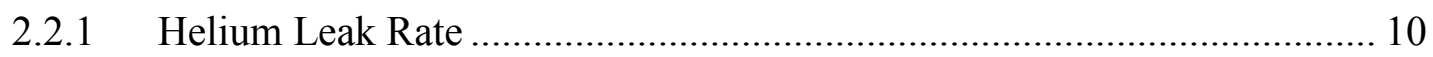

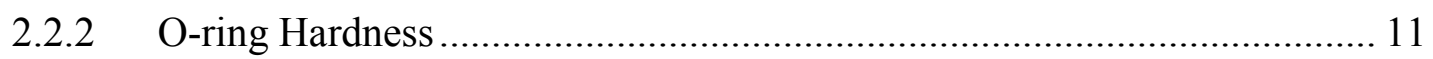

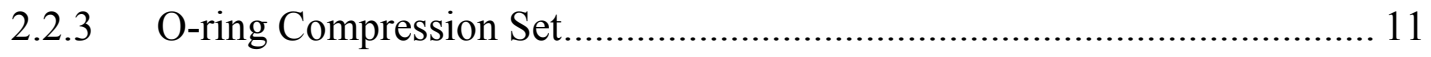

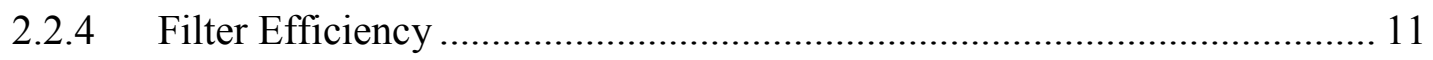

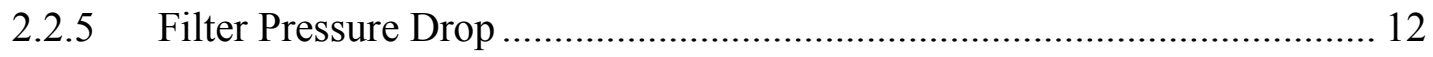

2.2.6 Water Penetration Testing................................................................ 12

2.2.7 Corrosion Evaluation ........................................................................ 12

3 Materials and Containers ………………………........................................ 13

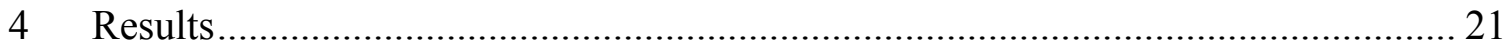

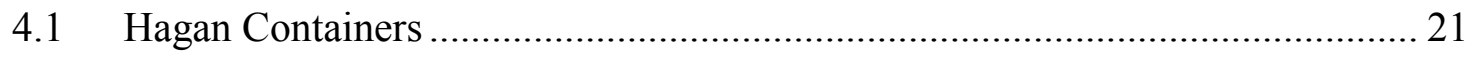

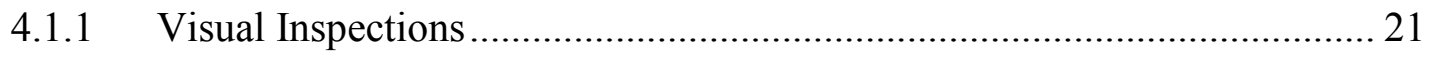

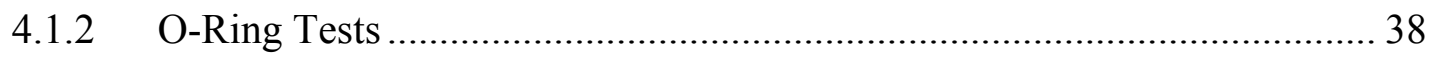

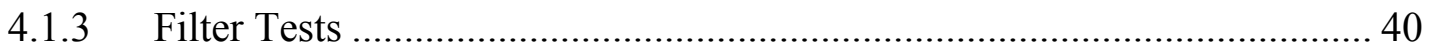

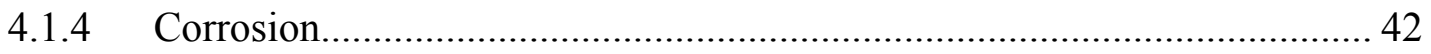

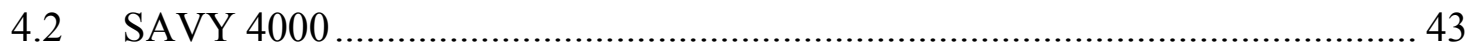

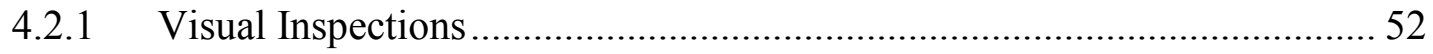

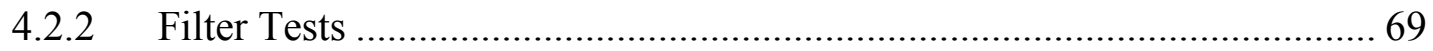

4.2.3 Annual Surveillance (NDE only) ............................................................ 72

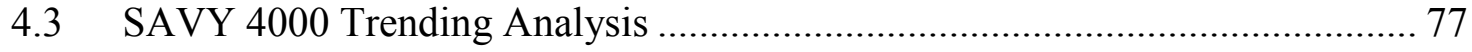

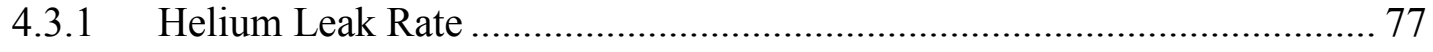

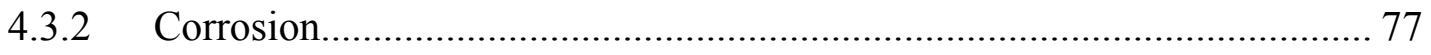

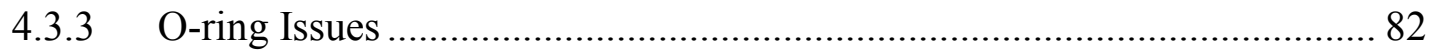

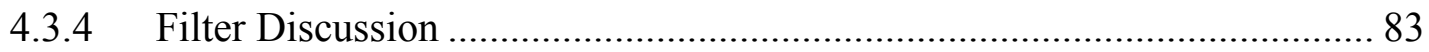




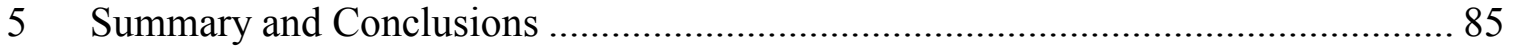

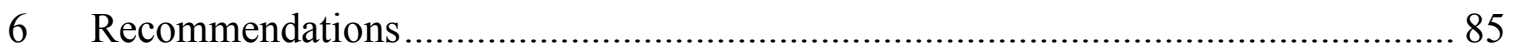

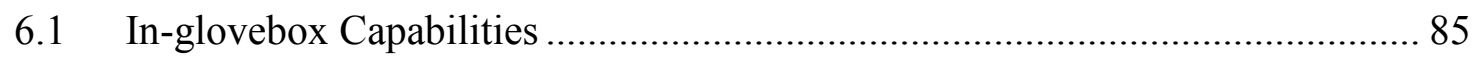

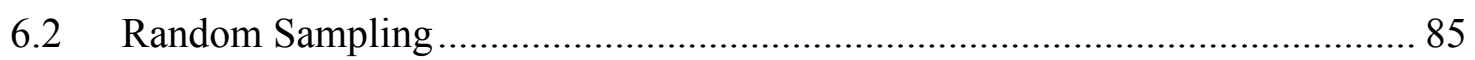

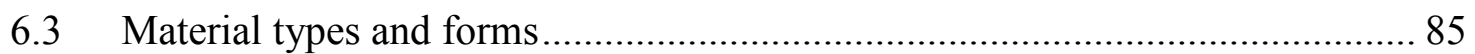

6.4 First Alternative Bag-out Bag Surveillance Item........................................... 86

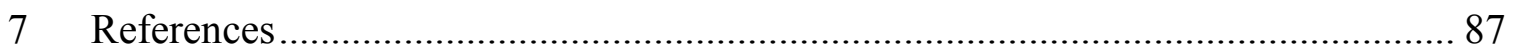




\section{Table of Figures}

Figure 4-1. Visual inspection of 18H1. (A) Corrosion on inside surfaces of Hagan container along weld and in areas associated with PVC tape handle. (B) Paint can with PVC tape handle. Spots of corrosion found on Hagan lid. (C) Condition of bagout bags. (D) Inside surfaces of the paint can that held the bagged item. .................. 26

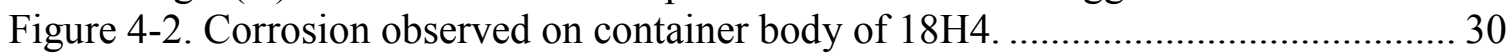

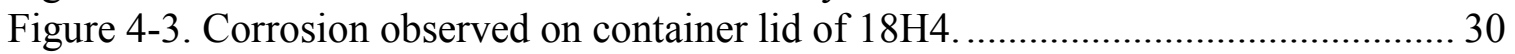

Figure 4-4. Photographs of $18 \mathrm{H} 7$ at unpacking. (A) External surfaces of container $18 \mathrm{H} 7$ showing white powder or etching on filter cap. (B) Inside surface of the lid covered in bag-out bag residue. Corrosion on threads shows gases escaped sealing surface. (C) Bag-out bag residue covering inside surface of the body. (D) Condition of bag-out bag.

Figure 4-5. Images of 18H7 showing corrosion on threads.......................................... 35

Figure 4-6. Eroded/pitted areas (showing as light spots) on the 18H7 Hagan lid........... 35

Figure 4-7. Magnified images of the eroded areas on container $18 \mathrm{H} 7$ bottom. .............. 36

Figure 4-8. Pits measured by optical microscopy on the $18 \mathrm{H} 7$ Hagan container lid........ 37

Figure 4-9. Leak rates measured for each Hagan container in FY16 (blue bars) FY17 (blue bars), and FY18 (orange bars). The red line shows the failure criteria. One container failed each in FY17 and FY18. Values on y-axis are in atmcc/sec.

Figure 4-10. Durometer measurements for each Hagan container in FY16 (blue bars) FY17 (blue bars), and FY18 (orange bars). The red and purple lines represent the lower and upper bounds for the hardness, respectively. The O-ring durometer values on the $\mathrm{y}$-axis are Shore-M hardness values.

Figure 4-11. Filter particle penetration measurements for each Hagan container measured in FY16 (blue bars) FY17 (blue bars), and FY18 (orange bars). The red line represents the upper bound for filter penetration. Values on $y$-axis are the filter efficiency....

Figure 4-12. Filter pressure drop measurements for each Hagan container measured in FY16 (blue bars) FY17 (blue bars), and FY18 (yellow bars). The red line represents the upper bound for filter penetration. Values on y-axis are pressure drop in units of inches W.C.

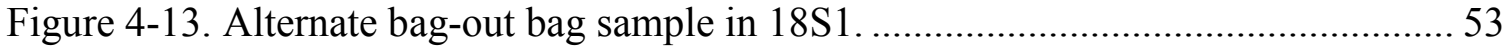

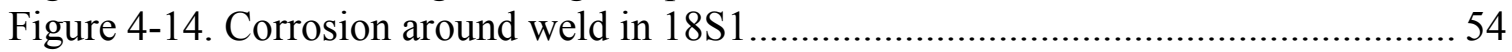

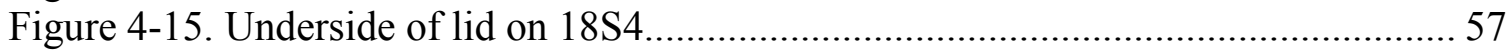

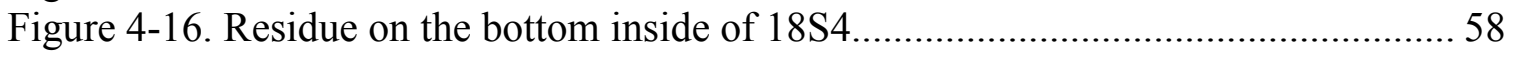

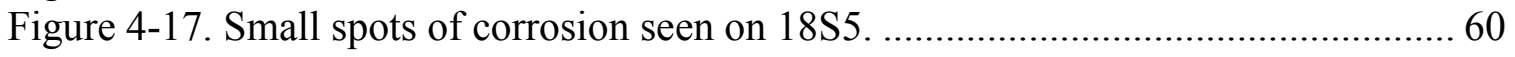

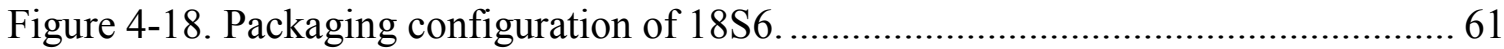

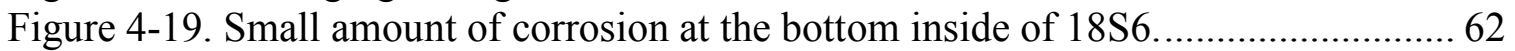

Figure 4-20. Small tear on the bottom of the bag-out bag that was in 18S6. ..................... 62

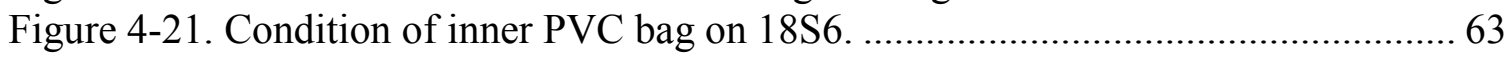

Figure 4-22. Leak rates for surveillance containers, measured for each container are shown as blue bars with the failure criteria shown with the orange line. .................. 66

Figure 4-23. Leak rates for transfer containers, measured for each container are shown as blue bars with the failure criteria shown with the orange line. 
Figure 4-24. Durometer measurements of the surveillance SAVY 4000 O-ring with the red and green and red bands showing the upper and lower failure limits..................6 68

Figure 4-25. Durometer measurements of the transfer container SAVY 4000 O-ring with the green and red bands showing the upper and lower failure limits........................6 69

Figure 4-26. Filter particle penetration measurements for storage containers................. 70

Figure 4-27. Filter particle penetration measurements for transfer containers................ 70

Figure 4-28. Filter pressure drop measurements for storage containers........................ 71

Figure 4-29. Filter pressure drop measurements for transfer containers. ........................ 71

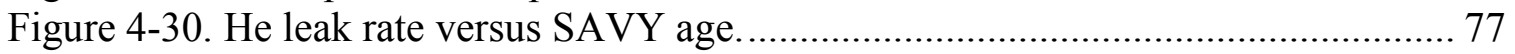

Figure 4-31. Durometer measurements versus SAVY age ...................................... 82

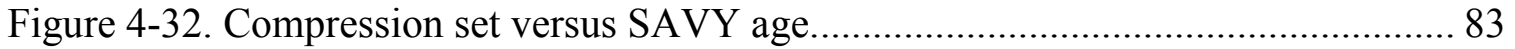

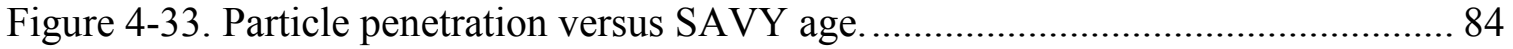

Figure 4-34. Filter pressure drop versus SAVY age ................................................. 84 


\section{Table of Tables}

Table 1. Numeric Ranking for General Corrosion in SAVY 4000 and Hagan Containers

Table 2. Roadmap for the Hagan and SAVY 4000 surveillance containers and their

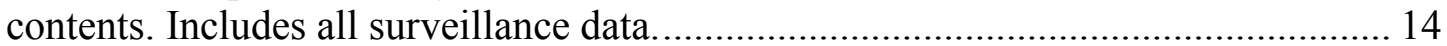

Table 3. Transfer containers tested in FY18 ............................................................... 20

Table 4. Surveillance test results for Hagan storage containers. ..................................... 22

Table 5. Unpacking data for Sample 18H1 (based on photographs) ............................. 25

Table 6. Unpacking data for Sample 18H2 (based on photographs) ............................. 27

Table 7. Unpacking data for Sample 18H3 (based on photographs) ............................... 28

Table 8. Unpacking data for Sample 18H4 (based on photographs) ............................. 29

Table 9. Unpacking data for Sample 18H5 (based on photographs) .............................. 31

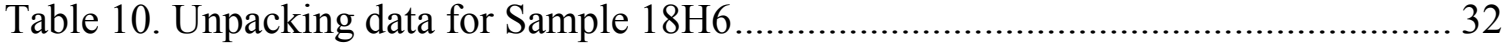

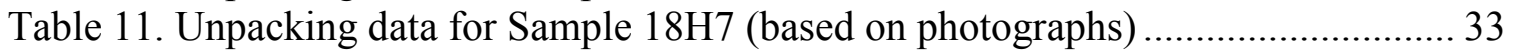

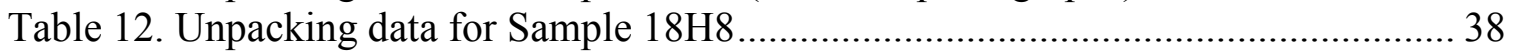

Table 13. Surveillance test results for SAVY 4000 storage containers .......................... 44

Table 14. Surveillance test results for SAVY 4000 transfer containers. ......................... 47

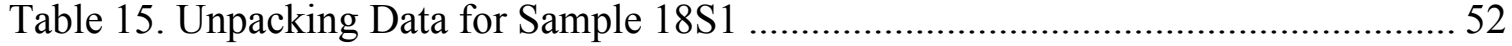

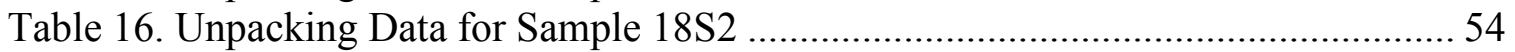

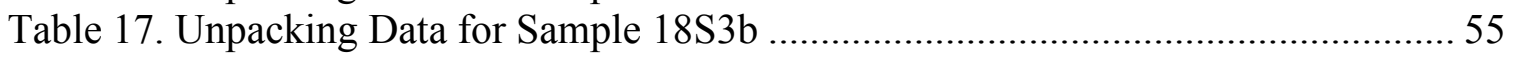

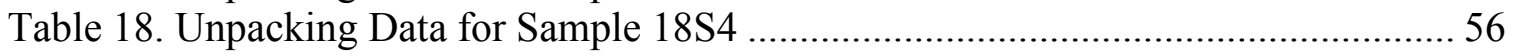

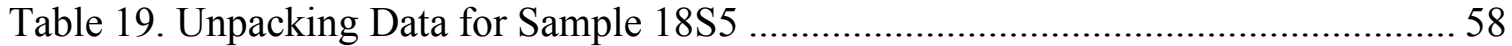

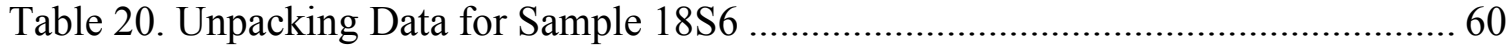

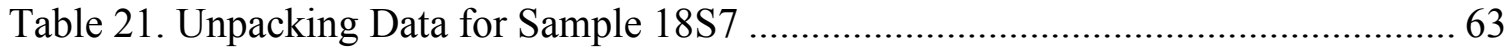

Table 22. Relevant data for annual measurements on the four NDE-only containers...... 73

Table 23. Relevant data for annual measurements on the four NDE-only containers..... 74

Table 24. Relevant data for annual measurements on the four NDE-only containers...... 75

Table 25. Relevant data for annual measurements on the four NDE-only containers...... 76 


\section{Introduction}

Approximately 1030 SAVY 4000 containers are in use at Los Alamos National Laboratory (LANL), and although Hagan containers are being phased out, there are still 3000 loaded Hagan containers in storage. The initial design lifetime for a SAVY 4000 container is 5 years, starting in April 2014. The lifetime extension program was initiated to determine how long a SAVY 4000 container may be used safely, potentially extending the design lifetime of the containers and avoiding unnecessary maintenance, replacement of containers or components, and handling of radioactive materials. The surveillance program was initiated to observe SAVY 4000 and Hagan containers during usage, and information from both surveillance and lifetime extension programs is integrated to build a comprehensive picture of the behavior of storage containers over time. At LANL, SAVY 4000 containers are designated as either storage containers or transfer containers as defined in TA55-DOP-091, "Nuclear Material Packaging." The primary difference is that storage containers are designed to function without maintenance over their entire design life, and transfer containers have a design life limited to 1 year and require an annual maintenance cycle to re-certify.

This work was performed for the surveillance program in accordance with the surveillance plan, "SAVY 4000 Field Surveillance Plan Update for 2018" [1]. The purpose of the surveillance plan and surveillance activities is to perform the following:

- Ensure that the containers currently in service are functioning properly

- Identify any unexpected problems in the containers or components

- Evaluate container component degradation over time against initial baseline measurements

- Contribute to the lifetime extension studies having met an initial goal of accumulating enough data within the initial 5-year design lifetime to extend the service lifetime of the SAVY 4000 container [5].

Containers with contents that represent upper bounding conditions have been selected for surveillance, as detailed in the surveillance plan [1]. This report includes surveillance testing results gathered over 1 year on a total of 36 SAVY 4000 containers ( 7 storage containers, and 21 transfer containers) and 8 Hagan containers. 


\section{Experimental}

This section describes the experimental tests on the SAVY 4000 storage and transfer containers and the Hagan containers, along with their respective O-rings and filters.

\subsection{Inspection}

\subsubsection{Container}

Inspection of the containers begins during unpacking and includes checking for external corrosion, evidence of pressurization, and dents or gouges that may have occurred during handling. The container is weighed, and a contamination survey is conducted before the container is opened. When the container is opened, the bag-out bag is inspected for signs of compromised integrity such as discoloration, brittleness, or the presence of liquid and the interior of the outer container is surveyed for contamination. The type of inner container is checked for appropriateness to the material form, and it is checked for significant corrosion or the presence of liquid. The visual inspection continues when the outer container is emptied and then released to the package-engineering team for further evaluation. The empty container is then checked for proper function of the closure mechanism, damage to the O-ring groove in the lid or on the body collar's sealing surface, filter discolorations or occlusions, and evidence of corrosion. If deemed necessary by a subject matter expert, the weld at the collar-body interface may be tested as well, but no welds were tested during the past years. The age of the container was calculated as the time between the initial packaging and the date of the surveillance measurements.

\subsubsection{O-ring}

Visual inspection of each O-ring was conducted according to PA-DOP-01080 Rev.1, "Surveillance Inspections of O-Rings for Nuclear Material Storage Containers," using a 4-inch, illuminated magnifying lens to look for O-ring defects such as flashing; mold mismatch; damage to the O-ring, such as cuts or abrasions; and the presence of dirt, hair, or dust on the O-ring. Irregularities were noted on the inspection sheet and corrected by cleaning, if possible.

\subsection{Tests}

\subsubsection{Helium Leak Rate}

The leak rate for each SAVY 4000 container with its original O-ring installed was measured in the inside-out mode using a LACO Flexstation ${ }^{\mathrm{TM}}$ bell-jar helium massspectroscopy leak tester according to the procedure in PA-AP-01158 Rev. 0, "Helium Leak Test Procedure of the SAVY 4000." The leak tester detects the presence of a leak by analyzing for helium leakage into a bell jar held near vacuum when the container is charged with 75 Torr of helium.

The O-ring passes the leak test if the measured leak rate is below a threshold value of $1 \times 10^{-5} \mathrm{~atm} \mathrm{cc} \mathrm{s}^{-1}$. That threshold value was determined for leak rate testing of SAVY 4000 container O-rings in Section 5.2 of the SAVY 4000 safety analysis report [6]. 
A single-point calibration is done before the measurements are taken each day. During FY17, the calibration standard used had a value of $1.05 \times 10^{-7} \mathrm{~atm}$. cc/s. This value is low enough to ensure that a leak at the threshold value will register on the leak detector, but it is high enough to be distinguishable from the typical background leakage rate.

The sensitivity of the measurement is limited by the quantity of helium in the ambient atmosphere, which can come from helium diffusing out of porous parts in the leak tester from prior tests. This helium contributes to an apparent background leakage rate. A background measurement was taken before each measurement.

\subsubsection{O-ring Hardness}

The hardness of each O-ring was measured by durometer, according to PA-DOP-01080 Rev. 1, "Surveillance Inspections of O-Rings for Nuclear Material Storage Containers," on the Shore-M scale. The hardness value for each O-ring was taken as the average of five durometer measurements taken at arbitrary positions around the whole O-ring. The calibration of the durometer is checked before and after each day of surveillance testing, using known calibration hardness standards.

\subsubsection{O-ring Compression Set}

The compression set of the O-rings used in the storage containers was estimated from a measurement of the O-ring thickness several weeks after they were removed from their containers as part of the SAVY 4000 surveillance program. The initial thickness of the Orings was $5.333 \pm 0.045 \mathrm{~mm}$, derived from the average of 85 unused O-rings, measured three times each. The compressed thickness was taken to be the gland depth of the container, which was determined by finding the best value for the difference between the lid diameter and the collar diameter, measured as part of the inspection process. If measurements were unavailable, the mean value for that particular size of container was taken from those surveyed in the receipt inspections. The final thickness measurement was taken by suspending the O-ring within the beam of a laser micrometer and averaging eight measurements at arbitrary positions around the O-ring. The uncertainty varied with the precision of the values available for each calculation, but generally was in the range of $5 \%-8 \%$.

\subsubsection{Filter Efficiency}

Each filter was subjected to particle penetration testing, in which the concentration of a test aerosol is measured downstream of the filter per PA-DOP-01580 Rev. 1. The test aerosol used was polyaphla-olefin with a concentration of $65 \pm 15 \mu \mathrm{g} / \mathrm{L}$ upstream of the filter. The concentration was measured using an Air Techniques International (ATI) $2 \mathrm{H}$ photometer, which was modified by ATI for the test flow rate of $200 \mathrm{cc} / \mathrm{min}$. The filters must capture at least $99.97 \%$ of the challenge aerosol. The development of this instrument and calibration information is discussed in LA-UR-16-20507, "Development and Use of a Low-Flow Filter Test System for the Filters Used in Special Nuclear Material Storage Containers". 


\subsubsection{Filter Pressure Drop}

Each filter was subjected to pressure drop testing per PA-DOP-01580 Rev. 1. The pressure drop tests were performed in conjunction with the aerosol tests. After the upstream aerosol concentration is measured, the instrument is switched to measure the concentration downstream of the filter, where the data system records 15 pressure drop data values in one-second intervals. The first two data points are discarded because there is an interval with a momentary spike in the system pressure. The pressure drop must be $<1$ inch water column at a flow rate of $200 \mathrm{cc} / \mathrm{min}$ to be considered passing.

\subsubsection{Water Penetration Testing}

A selection of SAVY 4000 containers underwent water penetration testing using PADOP-01768 Rev. 1, "Surveillance Inspections of Filter Water Resistance". The water penetration testing is a new capability added this year. A pressure of water at 12 " water column was applied to the filter on the outside of the lid and the pressure was held for 1 minute. The opposite side of the filter was monitored during the 1-minute interval to check for any water penetration. If no penetration was observed, the filter was considered to have passed the test. This test is comparable to the water penetration test performed at the time of container manufacture. Due to additional logistical considerations and time constraints associated with water penetration testing, the focus of testing this FY was on SAVY 4000 containers due to a concern that the filter membrane could degrade with exposure to high wattage and gamma radiation.

\subsubsection{Corrosion Evaluation}

The visual inspection of SAVY 4000 and Hagan containers includes an evaluation of the corrosion found on container surfaces. Any corrosion observed during the visual inspection is documented and photographed. In an effort to better understand the conditions under which corrosion occurs, a numeric ranking was assigned to each surveillance container from FY13 through FY18. The numeric ranking describes the severity of the corrosion based on its overall appearance with consideration for the relative coverage and density of the corrosion. The numeric ranking ranges from 0 , which indicates no corrosion to a ranking of 3, which is the most severe. The ranking can be used to compare the corrosion behavior for various material forms and material types as well as to assess the progression of corrosion over time. The levels of numeric rankings are defined in Table 1 below. 
Table 1. Numeric Ranking for General Corrosion in SAVY 4000 and Hagan Containers

\begin{tabular}{|c|l|l|}
\hline $\begin{array}{c}\text { Numeric } \\
\text { Ranking }\end{array}$ & \multicolumn{1}{|c|}{ Description } & \multicolumn{1}{c|}{ Criteria } \\
\hline $\mathbf{0}$ & No Corrosion & $\begin{array}{l}\text { No corrosion, staining, spots, or coatings } \\
\text { observed }\end{array}$ \\
\hline $\mathbf{1}$ & $\begin{array}{l}\text { Isolated General } \\
\text { Corrosion }\end{array}$ & $\begin{array}{l}\text { Corrosion, staining, spots, or coatings } \\
\text { observed in isolated areas (e.g. corrosion } \\
\text { found on weld only) }\end{array}$ \\
\hline $\mathbf{2}$ & Light General Corrosion & $\begin{array}{l}\text { Corrosion, staining, spots, or coatings } \\
\text { throughout container; light in overall density; } \\
\text { bare metal visible }\end{array}$ \\
\hline $\mathbf{3}$ & Heavy General Corrosion & $\begin{array}{l}\text { Corrosion, staining, spots, or coatings } \\
\text { throughout container; heavy (dark) in overall } \\
\text { density; little or no bare metal visible }\end{array}$ \\
\hline
\end{tabular}

\section{Materials and Containers}

For FY2018 surveillance, the selection criteria were updated based on the corrosion identified in previous years. Containers were selected based on the material form (e.g., radiation, corrosive or gas generating potential), wattage (e.g., high Am), container size (e.g., $\leq 5 \mathrm{Qt}$ ) and the age (e.g., as old as possible) of the containers. The history of the material form was also considered, e.g., items were included which had previously been found to cause corrosion. The 2017 surveillance plan forward also added Hagan containers. These containers were added due to the age of the containers, the similar material of construction (304L for Hagan, 316L for SAVY 4000), and the fact that the containers are still in storage and likely will be for at least a decade. The 316L components of the SAVY 4000 containers are expected to be more corrosions resistant than 304L components of the Hagan containers.

Table 2 provides a roadmap of all the containers tested. Where available, the SAVY 4000 container number that Hagan material was transferred into is included. Also included in the table are the container ages and the thermal power. The plutonium mass ranges from $4 \mathrm{~g}$ to $2355.6 \mathrm{~g}$. The physical and chemical forms cover a broad range, including compounds ( $\mathrm{Pu}$ dioxide and $\mathrm{Pu}$ chloride), metals (unalloyed $\mathrm{Pu}$ metal), and process residues (filter residue, direct oxide reduction [DOR] salt, incinerator ash, ER salt, and molten salt extraction [MSE] salt). The isotopics of the materials range from weapons grade $\mathrm{Pu}$ (MT 52) to fuel grade $\mathrm{Pu}$ (MT 54-57), high americium items (MT 5X+44, MSE salts), and heat source $\mathrm{Pu}(\mathrm{MT} \mathrm{83})$. The thermal power ranges from $0 \mathrm{~W}$ to $19.7 \mathrm{~W}$. The age (time in service) of the Hagan containers ranges from 4.8 to 17.6 years, and the age of the SAVY 4000 containers ranges from 0.84 to 6.6 years (over the entire surveillance program). 
Table 2. Roadmap for the Hagan and SAVY 4000 surveillance containers and their contents. Includes all surveillance data.

\begin{tabular}{|c|c|c|c|c|c|c|c|c|c|c|}
\hline Surv \# & FY & Chemical Subform & $\begin{array}{l}\text { Material } \\
\text { Type }\end{array}$ & Material Name & Hagan SN & SAVY SN & $\begin{array}{c}\text { Pu-239 } \\
\text { (g) }\end{array}$ & $\begin{array}{c}\text { Container } \\
\text { Age (y) }\end{array}$ & $\begin{array}{l}\text { Thermal } \\
\text { Power } \\
\text { (W) }\end{array}$ & $\begin{array}{c}\text { Corrosion } \\
\text { Ranking }\end{array}$ \\
\hline $13 \mathrm{H} 1$ & 2013 & MSE Salt & $52+44$ & CAXBL128D & $\begin{array}{c}\text { 8/02 LANL-813, } \\
30142\end{array}$ & 111103026 & 835 & 8.55 & 13.4 & 2 \\
\hline $13 \mathrm{H} 2$ & 2013 & ER Salt & 52 & GBS005 & $\begin{array}{c}\text { 4/02 A-28, } \\
04 / 02-08028\end{array}$ & 041208025 & 1875 & 8.22 & 4.9 & 1 \\
\hline $13 \mathrm{H} 3$ & 2013 & ER Salt & 52 & GBS059 & $\begin{array}{l}\text { 4/02 A-207, } \\
04 / 02-08010\end{array}$ & 041208004 & 1877 & 8.22 & 4.9 & 2 \\
\hline $13 \mathrm{H} 4$ & 2013 & Incinerator Ash & 54 & INCA-20 & $\begin{array}{c}\text { 4/99 LANL-429, } \\
\text { 05/99 NMC } \\
08000-305\end{array}$ & 041208043 & 830 & 7.93 & 3.4 & 2 \\
\hline $13 \mathrm{H} 5$ & 2013 & Incinerator Ash & 54 & INCA-21 & $\begin{array}{c}\text { 4/99 LANL-405, } \\
\text { 04/02-08145 }\end{array}$ & 041208009 & 913 & 8.3 & 3.8 & 2 \\
\hline $13 \mathrm{H} 6$ & 2013 & $\mathrm{MgO}$ & 52 & ORF633956XBLC & $\begin{array}{c}\text { 2/99 LANL-83, } \\
80208\end{array}$ & 041208038 & 248 & 13.17 & 0.7 & $\mathrm{n} / \mathrm{a}$ \\
\hline $13 \mathrm{H} 7$ & 2013 & Salt & 52 & PCS68B1 & $\begin{array}{l}\text { 4/02 A-134, } \\
08 / 06-08077\end{array}$ & 041208028 & 811 & 4.82 & 2.1 & $\mathrm{n} / \mathrm{a}$ \\
\hline $13 \mathrm{H} 8$ & 2013 & Tetra-fluoride & 54 & PHX5R4 & $\begin{array}{c}\text { 8/05 LANL- } \\
2282,08 / 05- \\
03282\end{array}$ & 121103052 & 166 & 6.45 & 0.7 & 0 \\
\hline $13 \mathrm{H} 9$ & 2013 & Sweepings/Screenings & 52 & POX4275C1 & $\begin{array}{c}\text { 7/02 LANL-393, } \\
06 / 02-05183\end{array}$ & 091205182 & 1037 & 7.56 & 2.73 & 0 \\
\hline $13 \mathrm{H} 10$ & 2013 & Dioxide & 56 & RBXS5657-1A & $\begin{array}{c}\text { 7/02 LANL-515, } \\
06 / 02-05305\end{array}$ & 041205026 & 751 & 8.81 & 3.8 & 2 \\
\hline
\end{tabular}




\begin{tabular}{|c|c|c|c|c|c|c|c|c|c|c|}
\hline Surv \# & FY & Chemical Subform & $\begin{array}{l}\text { Material } \\
\text { Type }\end{array}$ & Material Name & Hagan SN & SAVY SN & $\begin{array}{c}\text { Pu-239 } \\
\text { (g) }\end{array}$ & $\begin{array}{l}\text { Container } \\
\text { Age }(y)\end{array}$ & $\begin{array}{l}\text { Thermal } \\
\text { Power } \\
\text { (W) }\end{array}$ & $\begin{array}{c}\text { Corrosion } \\
\text { Ranking }\end{array}$ \\
\hline $13 \mathrm{H} 11$ & 2013 & DOR Salt & 52 & SLTF3123A & $\begin{array}{c}\text { 8/99 LANL- } \\
1178,07 / 02- \\
03184\end{array}$ & 121103062 & 1900 & 7.84 & 5 & 0 \\
\hline $13 \mathrm{H} 12$ & 2013 & Salt & 52 & SWPVTB15 & $\begin{array}{c}\text { 08/02 LANL- } \\
897,08 / 05- \\
03300\end{array}$ & 111103001 & 678 & 5.77 & 1.8 & $\mathrm{n} / \mathrm{a}$ \\
\hline $13 \mathrm{H} 13$ & 2013 & Sweepings/Screenings & 52 & VTB-16C1 & $\begin{array}{l}\text { 4/02 A-164, } \\
04 / 02-05164\end{array}$ & 021205029 & 1013 & 7.49 & 2.7 & $\mathrm{n} / \mathrm{a}$ \\
\hline $13 \mathrm{H} 14$ & 2013 & $\mathrm{MgO}$ & 52 & XBLC9413 & $\begin{array}{c}\text { 2/99 LANL-80, } \\
80207\end{array}$ & 041208055 & 356 & 13.34 & 0.9 & $\mathrm{n} / \mathrm{a}$ \\
\hline $13 \mathrm{H} 15$ & 2013 & MSE Salt & $56+44$ & XBLS25 & $\begin{array}{c}\text { 10/99 LANL- } \\
1932,80234\end{array}$ & 041208031 & 430 & 7.84 & 12.4 & $\mathrm{n} / \mathrm{a}$ \\
\hline $13 \mathrm{H} 16$ & 2013 & DOR Salt & 52 & XBSOX153 & $\begin{array}{c}\text { 3/06 LANL-296, } \\
\text { 03/06-05296 }\end{array}$ & 091205175 & 1079 & 6.33 & 2.84 & 0 \\
\hline $15 \mathrm{H} 1$ & 2015 & Dioxide & 52 & MOX51T & $\begin{array}{c}\text { 8/99-LANL- } \\
1277,06 / 02- \\
05305\end{array}$ & 121103054 & 2355.6 & 14.43 & 6.2 & 2 \\
\hline $15 \mathrm{H} 2$ & 2015 & ER Salt & 52 & XBS9455 & $\begin{array}{c}\text { 5/01-LANL-53 } \\
\text { 08/99NMC03- } \\
000-125\end{array}$ & 121103078 & 382.5 & 12.36 & 1 & 1 \\
\hline $15 \mathrm{H} 3$ & 2015 & Dioxide & 57 & BLO-39-11-16 & 3/01-LANL-209, & 111308080 & 544 & 5.62 & 5 & 3 \\
\hline $15 \mathrm{H} 4$ & 2015 & Dioxide & 56 & RBXS5657-2A & $\begin{array}{l}\text { LANL-441, } \\
04 / 02-08049\end{array}$ & 081305197 & 2059 & 11.48 & 10.5 & 3 \\
\hline $16 \mathrm{H} 1$ & 2016 & Dioxide & $54+44$ & XBPS333 & N/A & N/A & 80 & 8.1 & 2.9 & 3 \\
\hline
\end{tabular}




\begin{tabular}{|c|c|c|c|c|c|c|c|c|c|c|}
\hline Surv \# & FY & Chemical Subform & $\begin{array}{l}\text { Material } \\
\text { Type }\end{array}$ & Material Name & Hagan SN & SAVY SN & $\begin{array}{l}\text { Pu-239 } \\
\text { (g) }\end{array}$ & $\begin{array}{l}\text { Container } \\
\text { Age (y) }\end{array}$ & $\begin{array}{l}\text { Thermal } \\
\text { Power } \\
\text { (W) }\end{array}$ & $\begin{array}{c}\text { Corrosion } \\
\text { Ranking }\end{array}$ \\
\hline $17 \mathrm{H} 1$ & 2017 & Chloride & 52 & ATLAMS1S1 & $\begin{array}{c}\text { 07/02-03259 } \\
\text { 8/02 LANL- } \\
1014\end{array}$ & N/A & 75 & 11.10 & 0.2 & 2 \\
\hline $17 \mathrm{H} 2$ & 2017 & Chloride & 52 & CASLT966 & $\begin{array}{l}\text { 0702-03261 } \\
\text { LANL1061 }\end{array}$ & N/A & 286 & 12.02 & 0.6 & 1 \\
\hline $17 \mathrm{H} 3$ & 2017 & Filter Residue & 52 & CXLRES091599 & $\begin{array}{c}\text { 05/99-NMC05- } \\
000-079, \text { LANL- } \\
45\end{array}$ & N/A & 75 & 17.58 & 0.4 & 0 \\
\hline $17 \mathrm{H} 4$ & 2017 & Incinerator Ash & 52 & ASHX09 & $\begin{array}{c}\text { 05/99-NMC03- } \\
000-192, \text { LANL- } \\
1344\end{array}$ & N/A & 4 & 16.16 & 0 & 0 \\
\hline $17 \mathrm{H} 5$ & 2017 & Dioxide & 83 & $\begin{array}{l}\text { 10/10-01076 } \\
(\mathrm{Pu} 238)\end{array}$ & $\begin{array}{l}\text { 10/10-01076 10- } \\
10 \text { LANL-418 }\end{array}$ & N/A & (blank) & 6.27 & 19.7 & 1 \\
\hline $17 \mathrm{H} 6$ & 2017 & ER Salt & 52 & XORER6SLT2 & $\begin{array}{c}\text { 0402-08245 } \\
\text { A245 }\end{array}$ & N/A & 457 & 11.63 & 1.2 & 0 \\
\hline $17 \mathrm{H} 7$ & 2017 & MSE Salt & 56 & XBLS8A & $\begin{array}{l}0805 / 03142 \\
\text { LANL2139 }\end{array}$ & N/A & 114 & 10.67 & 3.5 & 2 \\
\hline $17 \mathrm{H} 8$ & 2017 & MSE Salt & 52 & XBSOX448A & $\begin{array}{c}\text { 08/06-01050 } \\
06 / 00 \text { LANL-98 }\end{array}$ & $\mathrm{N} / \mathrm{A}$ & 56 & 17.41 & 0.9 & $\mathrm{n} / \mathrm{a}$ \\
\hline $18 \mathrm{H} 1$ & 2018 & $\begin{array}{c}\text { Residue; Incinerator } \\
\text { Ash }\end{array}$ & 83 & TDC175 & $\begin{array}{c}\text { 9/99 NMC05 } \\
\text { LANL-1707 } \\
\text { 9/99NMC05000- } \\
392\end{array}$ & N/A & $\mathrm{N} / \mathrm{A}$ & 17 & 10.3 & 1 \\
\hline $18 \mathrm{H} 2$ & 2018 & ER Salt & 52 & XBS9409 & 2/99 LANL-88 & N/A & 445 & 16.8 & 1.2 & 0 \\
\hline $18 \mathrm{H} 3$ & 2018 & ER Salt & 52 & XBS0C6 & $\begin{array}{c}\text { 2/99 LANL-35 } \\
080159\end{array}$ & $\mathrm{~N} / \mathrm{A}$ & 328 & 16.8 & 0.9 & 0 \\
\hline
\end{tabular}




\begin{tabular}{|c|c|c|c|c|c|c|c|c|c|c|}
\hline Surv \# & FY & Chemical Subform & $\begin{array}{l}\text { Material } \\
\text { Type }\end{array}$ & Material Name & Hagan SN & SAVY SN & $\begin{array}{c}\text { Pu-239 } \\
\text { (g) }\end{array}$ & $\begin{array}{l}\text { Container } \\
\text { Age (y) }\end{array}$ & $\begin{array}{l}\text { Thermal } \\
\text { Power } \\
\text { (W) }\end{array}$ & $\begin{array}{c}\text { Corrosion } \\
\text { Ranking }\end{array}$ \\
\hline $18 \mathrm{H} 4$ & 2018 & MSE Salt & 52 & XBLS1124 & $\begin{array}{c}\text { LANL } 120 \text { 3/06 } \\
03 / 06-05120\end{array}$ & $\mathrm{~N} / \mathrm{A}$ & 110 & 6.2 & 0.45 & 2 \\
\hline $18 \mathrm{H} 5$ & 2018 & MgO Crucible & 52 & XBLC6380 & $\begin{array}{c}\text { 4/02 A-91 } \\
04 / 02-08091\end{array}$ & N/A & 210 & 10.5 & 0.6 & 0 \\
\hline $18 \mathrm{H} 6$ & 2018 & DOR Salt & 52 & SLT1802 & $\begin{array}{c}\text { 10/99 LANL- } \\
1987 \\
8 Q t-70\end{array}$ & $\mathrm{~N} / \mathrm{A}$ & 209 & 16 & 0.6 & 1 \\
\hline $18 \mathrm{H} 7$ & 2018 & MSE Salt & 52 & XBLSCL4051203 & $\begin{array}{c}\text { 8/02 LANL-763 } \\
08 / 02-01163\end{array}$ & N/A & 451 & 13 & 6.9 & 3 \\
\hline $18 \mathrm{H} 8$ & 2018 & MSE Salt & 52 & XBLSCL1210 & $\begin{array}{c}\text { 10/10 LANL- } \\
204 \\
10 / 10-03064\end{array}$ & N/A & 432 & 6 & 6.9 & 0 \\
\hline $15 \mathrm{~S} 1$ & 2015 & Dioxide & 52 & CXLOX082911 & $\mathrm{N} / \mathrm{A}$ & 031105052 & 786.9 & 3.03 & 2.07 & 0 \\
\hline $15 \mathrm{~S} 2$ & 2015 & MSE Salt & $52+44$ & XBLSCL1217 & $\mathrm{N} / \mathrm{A}$ & 121103083 & 178.5 & 1.86 & 2.85 & 0 \\
\hline $15 \mathrm{~S} 3$ & 2015 & Unalloyed Metal & 53 & XAP6 & $\mathrm{N} / \mathrm{A}$ & 031105002 & 69.5 & 3.01 & 0.21 & 0 \\
\hline $15 \mathrm{~S} 4$ & 2015 & Filter Residue & 52 & ROTRBJ-1C1 & $\mathrm{N} / \mathrm{A}$ & 031105051 & 452 & 3.46 & 1.19 & 0 \\
\hline $15 \mathrm{~S} 5$ & 2015 & $\mathrm{MgO}$ & 52 & XBLC9413 & $\begin{array}{c}\text { 2/99 LANL-80, } \\
80207\end{array}$ & 041208055 & 913 & 1.96 & 2.4 & 0 \\
\hline $15 \mathrm{~S} 6$ & 2015 & Tetra-fluoride & 54 & PHX3F & $\mathrm{N} / \mathrm{A}$ & 121103121 & 479.2 & 1.8 & 1.98 & 0 \\
\hline $15 \mathrm{~S} 7$ & 2015 & Tetra-fluoride & 54 & PHX5R4 & $\mathrm{N} / \mathrm{A}$ & 121103052 & 166 & 2.37 & 0.7 & 0 \\
\hline $15 \mathrm{~S} 8$ & 2015 & MSE Salt & $56+44$ & XBLS25 & $\begin{array}{c}\text { 10/99 LANL- } \\
1932,80234\end{array}$ & 041208031 & 430 & 2.55 & 12.4 & 0 \\
\hline $15 \mathrm{~S} 9$ & 2015 & Dioxide & 54 & $\mathrm{PBO}$ & $\mathrm{N} / \mathrm{A}$ & 031105028 & 1330.8 & 3.57 & 5.5 & 2 \\
\hline
\end{tabular}




\begin{tabular}{|c|c|c|c|c|c|c|c|c|c|c|}
\hline Surv \# & FY & Chemical Subform & $\begin{array}{l}\text { Material } \\
\text { Type }\end{array}$ & Material Name & Hagan SN & SAVY SN & $\begin{array}{c}\text { Pu-239 } \\
\text { (g) }\end{array}$ & $\begin{array}{l}\text { Container } \\
\text { Age }(y)\end{array}$ & $\begin{array}{c}\text { Thermal } \\
\text { Power } \\
\text { (W) }\end{array}$ & $\begin{array}{c}\text { Corrosion } \\
\text { Ranking }\end{array}$ \\
\hline $15 \mathrm{~S} 10$ & 2015 & Dioxide & 56 & RBXS5657-1A & $\begin{array}{c}\text { 7/02 LANL-515, } \\
06 / 02-05305\end{array}$ & 041205026 & 751 & 2.65 & 3.8 & 2 \\
\hline $16 \mathrm{~S} 1$ & 2016 & Non-Actinide M etal & 53 & SCRES65B & $\mathrm{N} / \mathrm{A}$ & 031105064 & 718 & 4.18 & 2.2 & 0 \\
\hline $16 \mathrm{~S} 2$ & 2016 & DOR Salt & 52 & SLT1303 & $\mathrm{N} / \mathrm{A}$ & 041208034 & 391.9 & 3.08 & 1 & 0 \\
\hline $16 \mathrm{~S} 3$ & 2016 & Unalloyed Metal & 52 & PMP91308 & N/A & 111308040 & 3220.6 & 0.84 & 8.5 & 0 \\
\hline $16 \mathrm{~S} 4$ & 2016 & MSE Salt & $52+44$ & XBLSCL1120A & $\mathrm{N} / \mathrm{A}$ & 121103041 & 155.1 & 3.22 & 2.5 & 0 \\
\hline $16 \mathrm{~S} 5$ & 2016 & Filter Residue & 52 & ROTRB9C3 & $\mathrm{N} / \mathrm{A}$ & 031105039 & 500 & 4.87 & 1.3 & 0 \\
\hline $16 \mathrm{~S} 6$ & 2016 & MSE Salt & $52+44$ & XBLSCL1213 & $\mathrm{N} / \mathrm{A}$ & 021205021 & 377.03 & 3.24 & 6.03 & 2 \\
\hline $16 \mathrm{~S} 7$ & 2016 & Unalloyed Metal & 52 & ARIAAQ137 & N/A & 091205173 & 2456.68 & 3.32 & 6.5 & 0 \\
\hline $16 \mathrm{~S} 8$ & 2016 & Dioxide & $52+83$ & AAP02OX & N/A & 041208057 & 509.4 & 3.4 & 21.28 & 3 \\
\hline $16 \mathrm{~S} 9$ & 2016 & MSE Salt & $52+44$ & XBLSCL1302 & N/A & 121103057 & 199.32 & 3.4 & 3.2 & 0 \\
\hline $16 \mathrm{~S} 10$ & 2016 & MSE Salt & $52+44$ & XBLSCL1301 & $\mathrm{N} / \mathrm{A}$ & 121103044 & 192.6 & 3.4 & 3.1 & 0 \\
\hline $16 \mathrm{~S} 11$ & 2016 & Dioxide & 52 & CXLOX082911 & N/A & 031105052 & 786.9 & 4.14 & 2.07 & 0 \\
\hline $16 \mathrm{~S} 12$ & 2016 & Unalloyed Metal & 53 & XAP6 & $\mathrm{N} / \mathrm{A}$ & 031105002 & 69.5 & 4.12 & 0.21 & 0 \\
\hline $16 \mathrm{~S} 13$ & 2016 & Filter Residue & 52 & ROTRBJ-1C1 & N/A & 031105051 & 452 & 4.58 & 1.19 & 0 \\
\hline $16 \mathrm{~S} 14$ & 2016 & MSE Salt & $52+44$ & XBLSCL1217 & N/A & 121103083 & 178.5 & 2.96 & 2.85 & 0 \\
\hline $16 \mathrm{~S} 15$ & 2016 & Dioxide & 83 & GPHS & N/A & 071201061 & 120 & 2.76 & 60.49 & 0 \\
\hline $17 \mathrm{~S} 1$ & 2017 & Dioxide & 57 & BLO-39-11-16 & N/A & 111308080 & 544 & 1.61 & 5 & 2 \\
\hline $17 \mathrm{~S} 2$ & 2017 & Dioxide & 52 & CXLOX082911 & N/A & 031105052 & 786.9 & 5.16 & 2.07 & 0 \\
\hline $17 \mathrm{~S} 3$ & 2017 & Unalloyed Metal & 53 & XAP6 & $\mathrm{N} / \mathrm{A}$ & 031105002 & 69.5 & 5.15 & 0.21 & 1 \\
\hline $17 \mathrm{~S} 4$ & 2017 & Filter Residue & 52 & ROTRBJ-1C1 & $\mathrm{N} / \mathrm{A}$ & 031105051 & 452 & 5.59 & 1.19 & 1 \\
\hline
\end{tabular}




\begin{tabular}{|c|c|c|c|c|c|c|c|c|c|c|}
\hline Surv \# & FY & Chemical Subform & $\begin{array}{c}\text { Material } \\
\text { Type }\end{array}$ & Material Name & Hagan SN & SAVY SN & $\begin{array}{c}\text { Pu-239 } \\
\text { (g) }\end{array}$ & $\begin{array}{c}\text { Container } \\
\text { Age }(y)\end{array}$ & $\begin{array}{l}\text { Thermal } \\
\text { Power } \\
\text { (W) }\end{array}$ & $\begin{array}{c}\text { Corrosion } \\
\text { Ranking }\end{array}$ \\
\hline $17 \mathrm{~S} 5$ & 2017 & MSE Salt & $52+44$ & XBLSCL1217 & $\mathrm{N} / \mathrm{A}$ & 121103083 & 178.5 & 3.98 & 2.895 & 1 \\
\hline 17S6 & 2017 & DOR Salt & 52 & SLT1303 & N/A & 111308050 & 391.9 & 1.03 & 1 & 0 \\
\hline $17 \mathrm{~S} 7$ & 2017 & Incinerator Ash & $52+54$ & INC20602 & N/A & 111103020 & 31 & 4.67 & 0.1 & 0 \\
\hline $17 \mathrm{~S} 8$ & 2017 & Unalloyed Metal & 53 & XAP6 (outer) & N/A & 111308059 & 69.5 & 1.02 & 0.21 & 0 \\
\hline $18 \mathrm{~S} 1$ & 2018 & Compound; Dioxide & 57 & BLO-39-11-16 & N/A & 111308080 & 544 & 2.4 & 5 & 2 \\
\hline $18 \mathrm{~S} 2$ & 2018 & Compound Residue & 52 & CXLOX082911 & N/A & 031105052 & 787 & 6.1 & 0.1 & 0 \\
\hline $18 \mathrm{~S} 3 \mathrm{~b}$ & 2018 & Unalloyed Metal & 53 & XAP6 & N/A & 111308059 & 69 & 2 & 0.21 & 0 \\
\hline $18 \mathrm{~S} 4$ & 2018 & Dioxide & 52 & ROTRBJ-1C1 & $\mathrm{N} / \mathrm{A}$ & 031105051 & 452 & 6.6 & 1.2 & 1 \\
\hline $18 \mathrm{~S} 5$ & 2018 & MSE Salt & $52+44$ & XBLSCL1217 & $\mathrm{N} / \mathrm{A}$ & 121103083 & 178 & 4.9 & 2.9 & 1 \\
\hline $18 \mathrm{~S} 6$ & 2018 & DOR Salt & 52 & SLTF3123A & N/A & 121103062 & 1900 & 5.4 & 2.9 & 1 \\
\hline $18 \mathrm{~S} 7$ & 2018 & Dioxide & 83 & SAMPCAN2 & N/A & 091205130 & $\mathrm{~N} / \mathrm{A}$ & 3.8 & 16.6 & 2 \\
\hline
\end{tabular}


Transfer containers are also subject to surveillance activities throughout their maintenance cycle, and although they are not surveillance items "per se" the inspection and test data is documented at the end of each FY. Transfer containers are intended primarily for transfer of nuclear material through the facility and they may be stored for up to one year, at which point the containers must be maintained testing before further use. The 21 SAVY 4000 transfer containers that have been maintenance cycled this FY are listed in Table 3 . The age of transfer containers from the time of maintenance ranges from 0 to 4 years. This age includes the time-period between when the container is emptied and when the maintenance is actually performed. Some of the containers are on the second or third maintenance cycle this fiscal year. Due to the way transfer containers are utilized, information about contents, dates of packaging, etc., is not recorded.

Table 3. Transfer containers tested in FY18.

\begin{tabular}{|c|c|c|c|c|c|}
\hline $\begin{array}{c}\text { Transfer } \\
\text { Container } \\
\text { Sample } \\
\text { Number }\end{array}$ & $\begin{array}{c}\text { SAVY } 4000 \text { Serial } \\
\text { Number }\end{array}$ & $\begin{array}{c}\text { Date of } \\
\text { Transfer } \\
\text { Container } \\
\text { Creation }\end{array}$ & $\begin{array}{l}\text { Date of latest } \\
\text { Maintenance }\end{array}$ & $\begin{array}{c}\text { Age at } \\
\text { Maintenance } \\
\text { ( years) }\end{array}$ & $\begin{array}{c}\text { Number of } \\
\text { Maintenance } \\
\text { cycles }\end{array}$ \\
\hline $1 \mathrm{~T}$ & $011701145 \mathrm{~B} / \mathrm{L}$ & $8 / 16 / 18$ & $8 / 16 / 18$ & 0 & 1 \\
\hline $2 \mathrm{~T}$ & 031403046B/L & $3 / 13 / 15$ & $8 / 8 / 18$ & 3.4 & 1 \\
\hline $3 T$ & 031403054B/L & $3 / 13 / 15$ & $8 / 8 / 18$ & 3.4 & 1 \\
\hline $4 \mathrm{~T}$ & $031403160 \mathrm{~B} / \mathrm{L}$ & $6 / 16 / 15$ & $7 / 19 / 18$ & 3.1 & 2 \\
\hline $5 T$ & 031403088B/L & $12 / 4 / 15$ & $7 / 19 / 18$ & 2.6 & 2 \\
\hline 6T & $071201136 \mathrm{~B} / \mathrm{L}$ & $5 / 5 / 14$ & $6 / 7 / 18$ & 4.0 & 2 \\
\hline $7 \mathrm{~T}$ & 071203168B/071203190L & $11 / 4 / 15$ & $6 / 7 / 18$ & 2.6 & 1 \\
\hline $8 \mathrm{~T}$ & 081301145B/L & $8 / 5 / 14$ & $7 / 19 / 18$ & 3.9 & 3 \\
\hline 9T & $081305013 \mathrm{~B} / \mathrm{L}$ & $11 / 24 / 14$ & $8 / 8 / 18$ & 3.7 & 2 \\
\hline 10T & 081305044B/L & $7 / 12 / 16$ & $8 / 8 / 18$ & 2.1 & 1 \\
\hline $11 \mathrm{~T}$ & 081305092B/L & $6 / 21 / 16$ & $7 / 19 / 18$ & 2.1 & 1 \\
\hline $12 \mathrm{~T}$ & $081305221 \mathrm{~B} / \mathrm{L}$ & $10 / 20 / 16$ & $6 / 7 / 18$ & 1.6 & 1 \\
\hline 13T & $111501011 \mathrm{~B} / \mathrm{L}$ & $9 / 28 / 16$ & $6 / 7 / 18$ & 1.7 & 1 \\
\hline $14 \mathrm{~T}$ & $111501014 \mathrm{~B} / 011701068 \mathrm{~L}$ & $9 / 28 / 16$ & $6 / 7 / 18$ & 1.7 & 1 \\
\hline $15 \mathrm{~T}$ & $111501076 \mathrm{~B} / \mathrm{L}$ & $9 / 28 / 16$ & $8 / 16 / 18$ & 1.9 & 1 \\
\hline $16 \mathrm{~T}$ & 031403066 & $3 / 25 / 15$ & $10 / 24 / 17$ & 2.6 & 1 \\
\hline 17T & 031403087 & $3 / 16 / 16$ & $10 / 31 / 17$ & 1.6 & 1 \\
\hline $18 \mathrm{~T}$ & 031403180 & $3 / 16 / 16$ & $10 / 31 / 17$ & 1.6 & 1 \\
\hline 19T & 041212006 & $3 / 30 / 16$ & $10 / 24 / 17$ & 1.6 & 1 \\
\hline 20T & 071201072 & $3 / 25 / 15$ & $10 / 24 / 17$ & 2.6 & 1 \\
\hline $21 \mathrm{~T}$ & 081305047 & $7 / 12 / 16$ & $10 / 24 / 17$ & 1.3 & 1 \\
\hline
\end{tabular}




\section{Results}

\subsection{Hagan Containers}

Surveillance was performed on 8 Hagan storage containers in FY18. The surveillance test results are given in Table 4. None of the containers had completed unpacking data forms, and the unpacking data presented in this section is derived from photographs and the container integrity inspection form (where available). Six of the containers were introduced into the glovebox line due to the concern of a having failed bag-out bag. Therefore, only two Hagan containers $18 \mathrm{H} 6$ and $18 \mathrm{H} 8$ had the filter particle penetration, filter pressure drop, O-ring durometer, and the helium leak tests performed. The surveillance results are presented below.

\subsubsection{Visual Inspections}

Corrosion was observed in four of the eight Hagan containers that had surveillance performed in FY18. Container $18 \mathrm{H} 1$ packaged with a $\mathrm{Pu}-238$ incinerator ash item had minor corrosion on the inside surfaces of the container resulting from the thermal degradation of PVC tape. Container 18H4 packaged with a MSE salt item had large areas of general corrosion likely from the degradation of the PVC bag. Container 18H6 packaged with MT52 DOR Salt had a strong chlorine smell when opened and had a small area on the container bottom with the appearance of being wet. Small, isolated spots of corrosion were found on the sidewall. However, the worst corrosion observed to date in a Hagan container was observed in container $18 \mathrm{H} 7$ that had to be cut open because of corroded threads on the lid and body, which indicate that corrosive gases had escaped through the sealing surfaces.

\subsubsection{Hagan Container, Surveillance, Sample \#18H1, 9/99 NMC05 LANL-1707 9/99NMC05000-392, TDC175, MT83, 22 g Pu, R47, Residue; Incinerator Ash, $10.3 \mathrm{~W}, 17$ years}

Container $18 \mathrm{H} 1$ was packaged with MT83 incinerator ash. The material was packaged inside of a taped slip lid container. The slip lid container was packaged inside two layers of bags (the innermost bag was a PVC bag-out bag). The bagged container was packaged inside of a paint can sealed with PVC tape and had a PVC tape handle. The paint can was packaged directly inside the 8-Qt Hagan container. No shielding was used in the package. The taped slip lid was functioning as the inner container in this configuration, and the paint can was used for contamination control.

The Hagan container was introduced into the glovebox line due to concerns that the paint can was no longer contamination free on the outside. Photographs were taken in lieu of the formal visual inspection and are shown in Figure 1. There were no visible signs that the exterior of the container was corroded or that the container was not properly sealed. Corrosion was found on the inside surfaces of the Hagan container. The heaviest corrosion was along circumferential weld on the upper portion of the sidewall and on the sidewall adjacent to the ends of the PVC tape handle (Figure 4-1A). The Hagan lid also has small corrosion spots distributed across the entire inside surface of the lid (Figure 4-1B). 
Table 4. Surveillance test results for Hagan storage containers.

\begin{tabular}{|c|c|c|c|c|c|c|c|c|c|c|c|}
\hline ID & $\begin{array}{c}\text { Container Serial } \\
\text { No. } \\
\text { and Size }\end{array}$ & $\begin{array}{c}\text { Container } \\
\text { Visual } \\
\text { Inspection }\end{array}$ & $\begin{array}{c}\text { Corrosion } \\
\text { Ranking }\end{array}$ & $\begin{array}{l}\text { O-Ring Visual } \\
\text { Inspection }\end{array}$ & $\begin{array}{c}\text { Material Name } \\
\text { and } \\
\text { IDC }\end{array}$ & $\begin{array}{c}\text { Sample } \\
\text { Power } \\
\text { (W) }\end{array}$ & $\begin{array}{l}\text { Package } \\
\text { Age } \\
\text { (y) }\end{array}$ & $\begin{array}{c}\text { Filter } \\
\text { Particle } \\
\text { Penetration } \\
\text { (\%) } \\
\pm \mathbf{0 . 0 0 0 2}\end{array}$ & $\begin{array}{l}\text { Filter } \\
\text { Pressure } \\
\text { Drop } \\
\text { (in W.C) } \\
\pm 0.02\end{array}$ & $\begin{array}{l}\text { Helium } \\
\text { Leak } \\
\text { Rate } \\
\left(\frac{a t m-c c}{s}\right)\end{array}$ & $\begin{array}{c}\text { O-ring } \\
\text { Durometer } \\
\text { (Shore M) } \\
\pm 2.15\end{array}$ \\
\hline $18 \mathrm{H} 1$ & $\begin{array}{c}\text { 9/99 NMC05 } \\
\text { LANL-1707 } \\
\text { 9/99NMC05000- } \\
392 \\
8 \text { Quart }\end{array}$ & $\begin{array}{l}\text { Spots of } \\
\text { corrosion on } \\
\text { inside of lid. } \\
\text { Corrosion } \\
\text { covering weld } \\
\text { on interior } \\
\text { surfaces (body } \\
\text { and wall) } \\
\text { associated } \\
\text { with tape on } \\
\text { inner } \\
\text { container }\end{array}$ & 1 & Not performed & $\begin{array}{c}\text { TDC175 } \\
\text { R47 }\end{array}$ & 10.3 & 17 & NM & NM & NM & NM \\
\hline $18 \mathrm{H} 2$ & $\begin{array}{c}\text { 2/99 LANL-88 } \\
8 \text { Quart }\end{array}$ & $\begin{array}{l}\text { Container in } \\
\text { good } \\
\text { condition. } \\
\text { No corrosion } \\
\text { observed. }\end{array}$ & 0 & Not performed & $\begin{array}{c}\text { XBS9409 } \\
\text { R65 }\end{array}$ & 1.2 & 16.8 & NM & NM & NM & NM \\
\hline $18 \mathrm{H} 3$ & $\begin{array}{c}\text { 2/99 LANL-35 } \\
080159 \\
8 \text { Quart }\end{array}$ & $\begin{array}{l}\text { Container in } \\
\text { good } \\
\text { condition. } \\
\text { No corrosion } \\
\text { observed. }\end{array}$ & 0 & Not performed & $\begin{array}{c}\text { XBS0C6 } \\
\text { R65 }\end{array}$ & 0.9 & 16.8 & NM & NM & NM & NM \\
\hline
\end{tabular}




\begin{tabular}{|c|c|c|c|c|c|c|c|c|c|c|c|}
\hline ID & $\begin{array}{c}\text { Container Serial } \\
\text { No. } \\
\text { and Size }\end{array}$ & $\begin{array}{l}\text { Container } \\
\text { Visual } \\
\text { Inspection }\end{array}$ & $\begin{array}{c}\text { Corrosion } \\
\text { Ranking }\end{array}$ & $\begin{array}{l}\text { O-Ring Visual } \\
\text { Inspection }\end{array}$ & $\begin{array}{c}\text { Material Name } \\
\text { and } \\
\text { IDC }\end{array}$ & $\begin{array}{c}\text { Sample } \\
\text { Power } \\
\text { (W) }\end{array}$ & $\begin{array}{c}\text { Package } \\
\text { Age } \\
\text { (y) }\end{array}$ & $\begin{array}{c}\text { Filter } \\
\text { Particle } \\
\text { Penetration } \\
(\%) \\
\pm \mathbf{0 . 0 0 0 2}\end{array}$ & $\begin{array}{c}\text { Filter } \\
\text { Pressure } \\
\text { Drop } \\
\text { (in W.C) } \\
\pm 0.02\end{array}$ & $\begin{array}{c}\text { Helium } \\
\text { Leak } \\
\text { Rate } \\
\left(\frac{a t m-c c}{s}\right)\end{array}$ & $\begin{array}{c}\text { O-ring } \\
\text { Durometer } \\
\text { (Shore M) } \\
\pm 2.15\end{array}$ \\
\hline $18 \mathrm{H} 4$ & $\begin{array}{l}\text { LANL } 120 \text { 3/06 } \\
\text { 03/ 06-05120 } \\
5 \text { Quart }\end{array}$ & $\begin{array}{l}\text { General } \\
\text { corrosion } \\
\text { covering large } \\
\text { areas of the } \\
\text { interior (body } \\
\text { and lid) }\end{array}$ & 2 & Not performed & $\begin{array}{c}\text { XBLS1124 } \\
\text { R83 }\end{array}$ & 0.45 & 6.2 & NM & NM & NM & NM \\
\hline $18 \mathrm{H} 5$ & $\begin{array}{c}\text { 4/02 A-91 } \\
\text { 04/ 02-08091 } \\
8 \text { Quart }\end{array}$ & $\begin{array}{l}\text { Container in } \\
\text { good } \\
\text { condition. } \\
\text { No corrosion } \\
\text { observed. }\end{array}$ & 0 & Not performed & $\begin{array}{c}\text { XBLC6380 } \\
\text { N50 }\end{array}$ & 0.6 & 10.5 & NM & NM & NM & NM \\
\hline $18 \mathrm{H} 6$ & $\begin{array}{c}\text { 10/99 LANL- } \\
1987 \\
8 Q t-70 \\
8 \text { Quart }\end{array}$ & $\begin{array}{l}\text { When opening } \\
\text { the container a } \\
\text { strong chlorine } \\
\text { smell was } \\
\text { noticed. There } \\
\text { is heat zone } \\
\text { radially } \\
\text { towards the } \\
\text { bottom of the } \\
\text { container. }\end{array}$ & 1 & No comments & $\begin{array}{c}\text { SLT1802 } \\
\text { R42 }\end{array}$ & 0.6 & 16 & $0.0086 \%$ & 0.868 & $1.80 \mathrm{E}-07$ & 79.9 \\
\hline
\end{tabular}




\begin{tabular}{|c|c|c|c|c|c|c|c|c|c|c|c|}
\hline ID & $\begin{array}{c}\text { Container Serial } \\
\text { No. } \\
\text { and Size }\end{array}$ & $\begin{array}{l}\text { Container } \\
\text { Visual } \\
\text { Inspection }\end{array}$ & $\begin{array}{c}\text { Corrosion } \\
\text { Ranking }\end{array}$ & $\begin{array}{l}\text { O-Ring Visual } \\
\text { Inspection }\end{array}$ & $\begin{array}{c}\text { Material Name } \\
\text { and } \\
\text { IDC }\end{array}$ & $\begin{array}{l}\text { Sample } \\
\text { Power } \\
\text { (W) }\end{array}$ & $\begin{array}{c}\text { Package } \\
\text { Age } \\
\text { (y) }\end{array}$ & $\begin{array}{c}\text { Filter } \\
\text { Particle } \\
\text { Penetration } \\
\quad(\%) \\
\pm \mathbf{0 . 0 0 0 2}\end{array}$ & $\begin{array}{l}\text { Filter } \\
\text { Pressure } \\
\text { Drop } \\
\text { (in W.C) } \\
\pm 0.02\end{array}$ & $\begin{array}{c}\text { Helium } \\
\text { Leak } \\
\text { Rate } \\
\left(\frac{a t m-c c}{s}\right)\end{array}$ & $\begin{array}{c}\text { O-ring } \\
\text { Durometer } \\
\text { (Shore M) } \\
\pm 2.15\end{array}$ \\
\hline $18 \mathrm{H} 7$ & $\begin{array}{c}\text { 8/02 LANL-763 } \\
\text { 08/ 02-01163 } \\
1 \text { Quart }\end{array}$ & & 3 & Not performed & $\begin{array}{c}\text { XBLSCL4051203 } \\
\text { R83 }\end{array}$ & 6.9 & 13 & NM & NM & NM & NM \\
\hline $18 \mathrm{H} 8$ & $\begin{array}{c}\text { 10/10 LANL-204 } \\
\text { 10/10-03064 } \\
3 \text { Quart }\end{array}$ & No comments & 0 & No comments & $\begin{array}{c}\text { XBLSCL1210 } \\
\text { R83 }\end{array}$ & 6.9 & 6 & $0.0118 \%$ & 0.797 & $\begin{array}{l}\text { Fail } \\
\text { (Gross } \\
\text { leak) }\end{array}$ & 80.1 \\
\hline
\end{tabular}


The outside of the paint can did not show corrosion, but the inside surfaces were covered with residue (Figure 4-1D). The PVC bag itself was darkened and brittle (Figure 4-1). The inner container and taped lid were intact.

Table 5. Unpacking data for Sample $18 \mathrm{H} 1$ (based on photographs)

\begin{tabular}{|c|c|}
\hline Surveillance sample number & $18 \mathrm{H} 1$ \\
\hline Operator & Unknown \\
\hline Date & $5 / 30 / 18$ \\
\hline Contamination found outside Hagan & No \\
\hline Contamination found inside Hagan & Unknown \\
\hline Overall package weight & Not meas. \\
\hline Body serial & 9/99NMC05000-392 \\
\hline Lid serial & 9/99 NMC05 LANL-1707 \\
\hline LOT ID & TDC175 \\
\hline Lead internal shield & No \\
\hline Pewter outer shield & No \\
\hline Outer container condition & Good \\
\hline O-ring installed & Yes \\
\hline Bag-out bag present & Yes, inside inner can \\
\hline Failed bag-out bag & Yes \\
\hline Liquid observed & Yes \\
\hline Condition of old bag-out bag & Dark colored \\
\hline Type of inner & Paint can \\
\hline Condition of inner & $\begin{array}{l}\text { Outside good; inside completely } \\
\text { covered in bag residue }\end{array}$ \\
\hline Failed metal inner & No \\
\hline Bag-out bag replace & Yes \\
\hline Inner replace & Unknown \\
\hline Beta/gamma dose (at lid/collar of Hagan) & Not meas. \\
\hline Beta/gamma dose (at $30 \mathrm{~cm}$ ) & Not meas. \\
\hline Neutron dose & Not meas. \\
\hline Comments & None \\
\hline
\end{tabular}




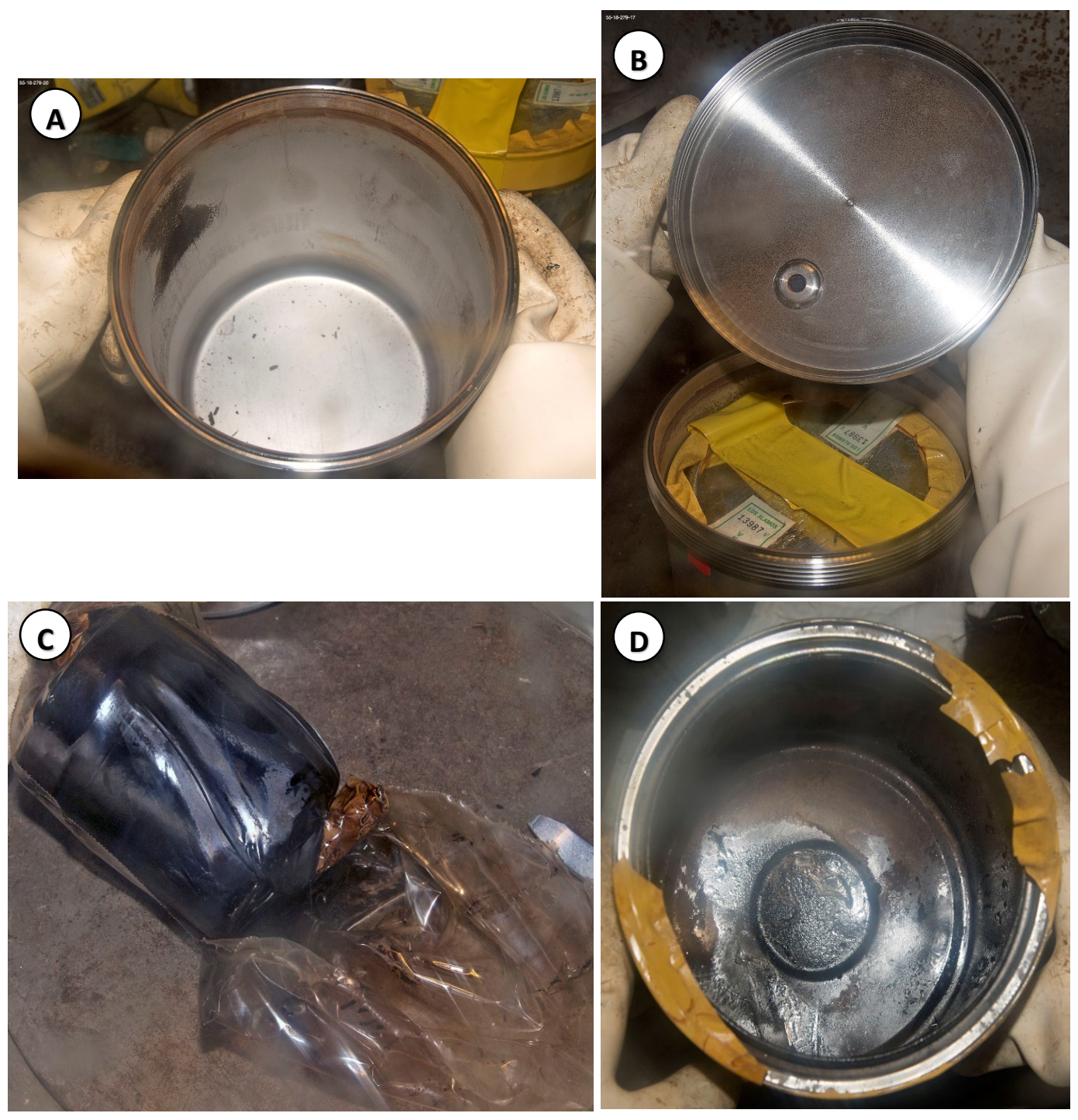

Figure 4-1. Visual inspection of 18H1. (A) Corrosion on inside surfaces of Hagan container along weld and in areas associated with PVC tape handle. (B) Paint can with PVC tape handle. Spots of corrosion found on Hagan lid. (C) Condition of bag-out bags. (D) Inside surfaces of the paint can that held the bagged item.

\subsubsection{Hagan Container, Surveillance, Sample \#18H2, 2/99 LANL-88, XBS9409, MT52, $445 \mathrm{~g}$ Pu, R65, ER Salt, $1.2 \mathrm{~W}, 16.8$ years}

Container $18 \mathrm{H} 2$ was packaged with MT52 ER salt. The material was packaged inside of a taped slip lid container. The slip lid container was packaged inside a single PVC bagout bag. The bagged container was packaged inside the 8-Qt Hagan container with lead shielding.

The Hagan container was introduced into the glovebox line due to concerns that the inner container no longer contamination free on the outside. Photographs were taken in lieu of the formal visual inspection. Based on the photographs, there were no visible signs that the exterior of the container was corroded or that the container was not properly sealed. 
Table 6. Unpacking data for Sample $18 \mathrm{H} 2$ (based on photographs)

\begin{tabular}{|c|c|}
\hline Surveillance sample number & $18 \mathrm{H} 2$ \\
\hline Operator & Unknown \\
\hline Date & $9 / 10 / 18$ \\
\hline Contamination found outside Hagan & No \\
\hline Contamination found inside Hagan & Unknown \\
\hline Overall package weight & Not meas. \\
\hline \multicolumn{2}{|l|}{ Body serial } \\
\hline Lid serial & 2/99 LANL-88 \\
\hline LOT ID & XBS9409 \\
\hline Lead internal shield & Yes \\
\hline Pewter outer shield & No \\
\hline Outer container condition & Good \\
\hline O-ring installed & Yes \\
\hline Bag-out bag present & Yes \\
\hline Failed bag-out bag & No \\
\hline Liquid observed & No \\
\hline Condition of old bag-out bag & Like new \\
\hline Type of inner & Slip lid \\
\hline Condition of inner & Good \\
\hline Failed metal inner & No \\
\hline Bag-out bag replace & Yes \\
\hline Inner replace & Unknown \\
\hline Beta/gamma dose (at lid/collar of Hagan) & Not meas. \\
\hline Beta/gamma dose $($ at $30 \mathrm{~cm})$ & Not meas. \\
\hline Neutron dose & Not meas. \\
\hline Comments & None \\
\hline
\end{tabular}

No corrosion was observed on the inside surfaces of the Hagan container. The bag-out bag showed little to no discoloration and was still intact. The inner container did not show corrosion on the exterior surface, and the PVC sealing tape was intact.

\subsubsection{Hagan Container, Surveillance, Sample \#18H3, 2/99 LANL-35 080159, XBSOC6, MT52, $328 \mathrm{~g}$ Pu, R65, ER Salt, $0.9 \mathrm{~W}, 16.8$ years}

Container $18 \mathrm{H} 3$ was packaged with MT52 ER salt. The material was packaged inside of a taped slip lid container. The slip lid container was packaged inside a single PVC bagout bag. The bagged container was packaged inside the 8-Qt Hagan container without shielding.

The Hagan container was introduced into the glovebox line due to concerns that the inner container no longer contamination free on the outside. Photographs were taken in lieu of 
the formal visual inspection. Based on the photographs, there were no visible signs that the exterior of the container was corroded or that the container was not properly sealed.

Table 7. Unpacking data for Sample $18 \mathrm{H} 3$ (based on photographs)

\begin{tabular}{|c|c|}
\hline Surveillance sample number & $18 \mathrm{H} 3$ \\
\hline Operator & Unknown \\
\hline Date & $9 / 10 / 18$ \\
\hline Contamination found outside Hagan & No \\
\hline Contamination found inside Hagan & Unknown \\
\hline Overall package weight & Not meas. \\
\hline Body serial & 080159 \\
\hline Lid serial & 2/99 LANL-35 \\
\hline LOT ID & XBS9409 \\
\hline Lead internal shield & No \\
\hline Pewter outer shield & No \\
\hline Outer container condition & Good \\
\hline O-ring installed & Yes \\
\hline Bag-out bag present & Yes \\
\hline Failed bag-out bag & No \\
\hline Liquid observed & No \\
\hline Condition of old bag-out bag & Like new \\
\hline Type of inner & Slip lid \\
\hline Condition of inner & Good \\
\hline Failed metal inner & No \\
\hline Bag-out bag replace & Yes \\
\hline Inner replace & Unknown \\
\hline Beta/gamma dose (at lid/collar of Hagan) & Not meas. \\
\hline Beta/gamma dose $($ at $30 \mathrm{~cm}$ ) & Not meas. \\
\hline Neutron dose & Not meas. \\
\hline Comments & None \\
\hline
\end{tabular}

No corrosion was observed on the inside surfaces of the Hagan container. The bag-out bag showed little to no discoloration and was still intact. The inner container did not show corrosion on the exterior surface, and the PVC sealing tape was intact.

\subsubsection{Hagan Container, Surveillance, Sample \#18H4, LANL 120 3/06 03/06-05120, XBLS1124, MT52, 110 g Pu, R83, MSE Salt, 0.5 W, 6.2 years}

Container $18 \mathrm{H} 4$ was packaged with MT52 MSE salt. The material was packaged inside of a taped slip lid container. The slip lid container was packaged inside a single PVC bagout bag. The bagged container was packaged inside the 5-Qt Hagan container without shielding. 
The Hagan container was introduced into the glovebox line due to concerns that the inner container no longer contamination free on the outside. Photographs were taken in lieu of the formal visual inspection. Based on the photographs, there were no visible signs that the exterior of the container was corroded or that the container was not properly sealed.

Corrosion was found on the inside surfaces of the Hagan container. The heaviest corrosion was along circumferential weld on the lower sidewall and bottom of the container. The corrosion appears to be heaviest in areas where the PVC bag was in contact with the inside of the container (Figure 4-2). The Hagan lid also has corrosion distributed across the most of the inside surface of the container lid (Figure 4-3). The bag-out bag showed some discoloration near the bottom of the bag but appeared to be intact.

Table 8. Unpacking data for Sample 18H4 (based on photographs)

\begin{tabular}{|c|c|}
\hline Surveillance sample number & $18 \mathrm{H} 4$ \\
\hline Operator & Unknown \\
\hline Date & $9 / 13 / 18$ \\
\hline Contamination found outside Hagan & No \\
\hline Contamination found inside Hagan & Unknown \\
\hline Overall package weight & Not meas. \\
\hline Body serial & $03 / 06-05120$ \\
\hline Lid serial & LANL 120 3/06 \\
\hline LOT ID & XBLS1124 \\
\hline Lead internal shield & No \\
\hline Pewter outer shield & No \\
\hline Outer container condition & Good \\
\hline O-ring installed & Yes \\
\hline Bag-out bag present & Yes \\
\hline Failed bag-out bag & No \\
\hline Liquid observed & No \\
\hline Condition of old bag-out bag & Good \\
\hline Type of inner & Slip lid \\
\hline Condition of inner & Good \\
\hline Failed metal inner & No \\
\hline Bag-out bag replace & Yes \\
\hline Inner replace & Unknown \\
\hline Beta/gamma dose (at lid/collar of Hagan) & Not meas. \\
\hline Beta/gamma dose (at $30 \mathrm{~cm}$ ) & Not meas. \\
\hline Neutron dose & Not meas. \\
\hline Comments & None \\
\hline
\end{tabular}




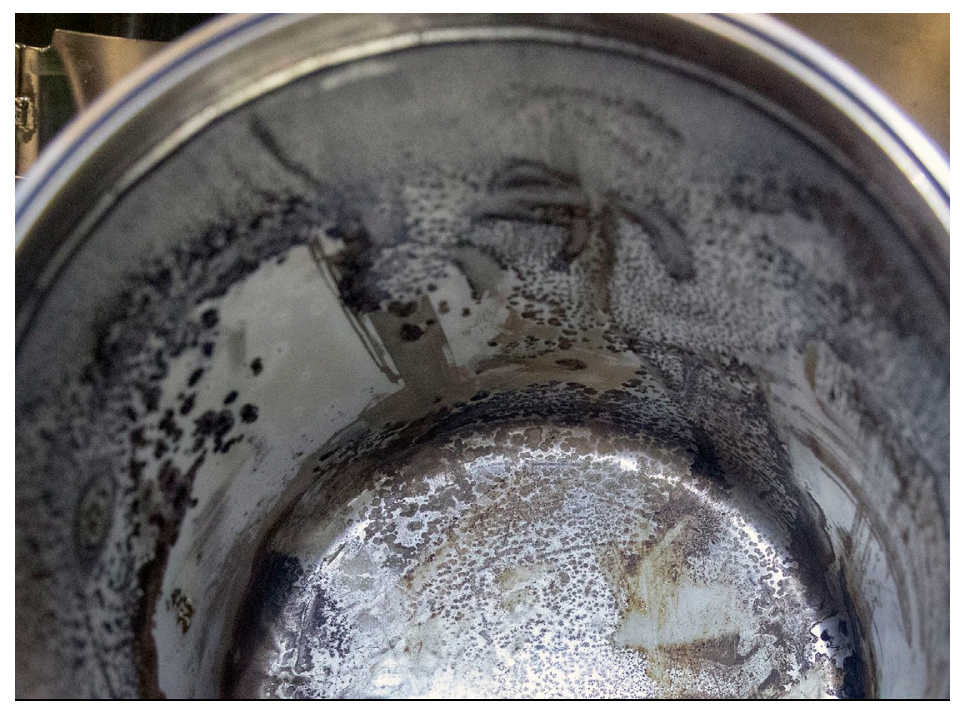

Figure 4-2. Corrosion observed on container body of $18 \mathrm{H} 4$.

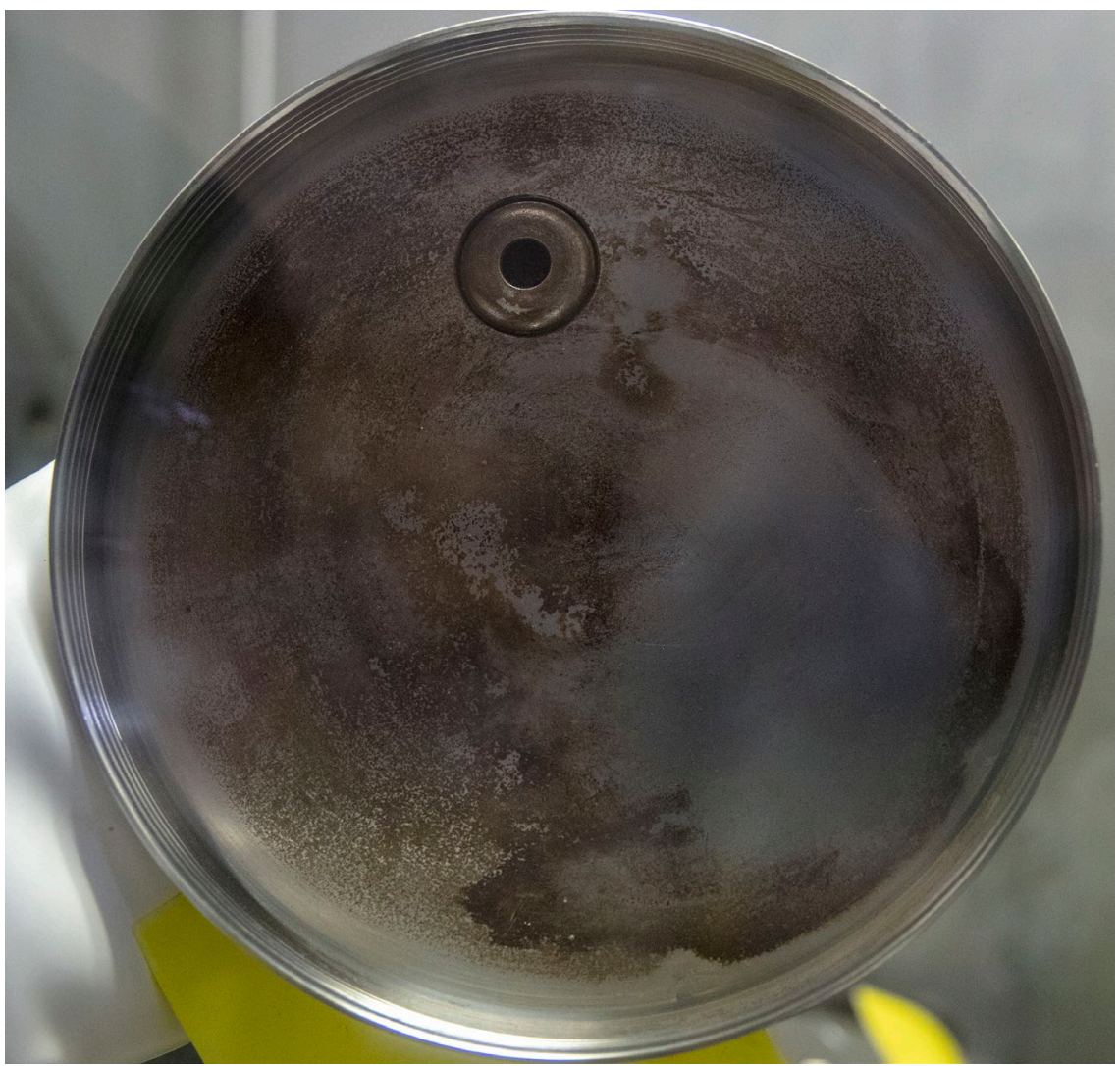

Figure 4-3. Corrosion observed on container lid of $18 \mathrm{H} 4$. 


\subsubsection{Hagan Container, Surveillance, Sample \#18H5, 4/02 A-91 04/02-08091, XBLC6380, MT52, $210 \mathrm{~g}$ Pu, N50, MgO Crucible, $0.6 \mathrm{~W}, 10.5$ years}

Container $18 \mathrm{H} 5$ was packaged with MT52 MgO crucible. The material was packaged inside of a taped slip lid container with a filter. The slip lid container was packaged inside a single PVC bag-out bag. The bagged container was packaged inside the 8-Qt Hagan container with external pewter shielding.

The Hagan container was introduced into the glovebox line due to concerns that the inner container no longer contamination free on the outside. Photographs taken in lieu of the formal visual inspection. Based on the photographs, there were no visible signs that the exterior of the container was corroded or that the container was not properly sealed.

Table 9. Unpacking data for Sample $18 \mathrm{H} 5$ (based on photographs)

\begin{tabular}{|c|c|}
\hline Surveillance sample number & $18 \mathrm{H} 5$ \\
\hline Operator & Unknown \\
\hline $\begin{array}{l}\text { Date } \\
\end{array}$ & $9 / 10 / 18$ \\
\hline Contamination found outside Hagan & No \\
\hline Contamination found inside Hagan & Unknown \\
\hline Overall package weight & Not meas. \\
\hline Body serial & $04 / 02-08091$ \\
\hline Lid serial & 4/02 A-91 \\
\hline LOT ID & XBLC6380 \\
\hline Lead internal shield & No \\
\hline Pewter outer shield & Yes \\
\hline Outer container condition & Good \\
\hline O-ring installed & Yes \\
\hline Bag-out bag present & Yes \\
\hline Failed bag-out bag & No \\
\hline Liquid observed & No \\
\hline Condition of old bag-out bag & Like new \\
\hline Type of inner & Slip lid \\
\hline Condition of inner & Good \\
\hline Failed metal inner & No \\
\hline Bag-out bag replace & Yes \\
\hline Inner replace & Unknown \\
\hline Beta/gamma dose (at lid/collar of Hagan) & Not meas. \\
\hline Beta/gamma dose (at $30 \mathrm{~cm}$ ) & Not meas. \\
\hline Neutron dose & Not meas. \\
\hline Comments & None \\
\hline
\end{tabular}


No corrosion was observed on the inside surfaces of the Hagan container. The bag-out bag showed little to no discoloration and was still intact. The inner container did not show corrosion on the exterior surface, and the PVC sealing tape was intact.

\subsubsection{Hagan Container, Surveillance, Sample \#18H6, 10/99 LANL-1987 8Qt-70 SLT1802, MT52, $209 \mathrm{~g}$ Pu, R42, DOR Salt, $0.6 \mathrm{~W}, 16$ years}

Container 18H6 was packaged with MT52 DOR Salt. The inside surfaces of the Hagan were contamination free. The container had a strong chlorine smell when opened and had a small area on the container bottom with the appearance of being wet. Small, isolated spots of corrosion were found on the sidewall, and staining was found on the container bottom. The condition of the bag-out bag and inner container are not known. The O-ring was inspected, and no issues were identified.

Table 10. Unpacking data for Sample $18 \mathrm{H} 6$

\begin{tabular}{|c|c|}
\hline Surveillance sample number & $18 \mathrm{H} 6$ \\
\hline Operator & Unknown \\
\hline Date & $6 / 29 / 18$ \\
\hline Contamination found outside Hagan & No \\
\hline Contamination found inside Hagan & No \\
\hline Overall package weight & Not meas. \\
\hline Body serial & 8Qt-70 \\
\hline Lid serial & 10/99 LANL-1987 \\
\hline LOT ID & SLT1802 \\
\hline Lead internal shield & Unknown \\
\hline Pewter outer shield & Unknown \\
\hline Outer container condition & Good \\
\hline O-ring installed & Yes \\
\hline Bag-out bag present & Yes \\
\hline Failed bag-out bag & No \\
\hline Liquid observed & No \\
\hline Condition of old bag-out bag & Unknown \\
\hline Type of inner & Unknown \\
\hline Condition of inner & Unknown \\
\hline Failed metal inner & No \\
\hline Bag-out bag replace & Unknown \\
\hline Inner replace & Unknown \\
\hline Beta/gamma dose (at lid/collar of Hagan) & Not meas. \\
\hline Beta/gamma dose (at $30 \mathrm{~cm}$ ) & Not meas. \\
\hline Neutron dose & Not meas. \\
\hline Comments & $\begin{array}{l}\text { When opening the container, a } \\
\text { strong chlorine smell was noticed. } \\
\text { There is heat zone radially towards } \\
\text { the bottom of the container. }\end{array}$ \\
\hline
\end{tabular}


Container $18 \mathrm{H} 6$ had the filter particle penetration, filter pressure drop, O-ring durometer, and the helium leak tests performed. No issues were identified during testing.

\subsubsection{Hagan Container, Surveillance, Sample \#18H7, 8/02-01163 LANL-763, XBLS4051203, MT52, 451 g Pu, R83, MSE Salt , $7 \mathrm{~W}, 13$ years}

Container $18 \mathrm{H} 7$ was packaged with MT52 MSE salt. The material was packaged inside of a taped slip lid container. The slip lid container was packaged inside a single PVC bagout bag. The bagged container was packaged inside the 1-Qt Hagan container with outer pewter shielding.

Table 11. Unpacking data for Sample $18 \mathrm{H} 7$ (based on photographs)

\begin{tabular}{|c|c|}
\hline Surveillance sample number & $18 \mathrm{H7}$ \\
\hline Operator & Kennard Wilson \\
\hline Date & $6 / 21 / 18$ \\
\hline Contamination found outside Hagan & No \\
\hline Contamination found inside Hagan & Unknown \\
\hline Overall package weight & Not meas. \\
\hline Body serial & $08 / 02-01163$ \\
\hline Lid serial & LANL-763 \\
\hline LOT ID & XBLSCL4051203 \\
\hline Lead internal shield & No \\
\hline Pewter outer shield & Yes \\
\hline Outer container condition & $\begin{array}{c}\text { Corrosion past sealing surface } \\
\text { Heavy coating of bag residue } \\
\text { Pitting observed }\end{array}$ \\
\hline O-ring installed & Yes \\
\hline Bag-out bag present & Yes \\
\hline Failed bag-out bag & No \\
\hline Liquid observed & No \\
\hline Condition of old bag-out bag & $\begin{array}{l}\text { Discolored; degraded; } \\
\text { liquid observed }\end{array}$ \\
\hline Type of inner & Slip lid \\
\hline Condition of inner & Good \\
\hline Failed metal inner & No \\
\hline Bag-out bag replace & $\mathrm{N} / \mathrm{A}$; item processed \\
\hline Inner replace & $\mathrm{N} / \mathrm{A}$; item processed \\
\hline Beta/gamma dose (at lid/collar of Hagan) & Not meas. \\
\hline Beta/gamma dose (at $30 \mathrm{~cm}$ ) & Not meas. \\
\hline Neutron dose & Not meas. \\
\hline Comments & None \\
\hline
\end{tabular}


This container was chosen for surveillance in FY17, but surveillance could not be performed because the lid became fixed to the body during storage. In the interim, the Hagan was overpacked in a 3-Qt SAVY 4000 container for 14 months. In June 2018, the SAVY 4000 was unloaded, and the Hagan container was introduced into the glovebox line for opening. (It was noted at that corrosion was found on the inside surfaces of the SAVY 4000 container used to overpack the 1-Qt Hagan. Photos and test results will be included in the FY19 Surveillance Report). The lid was removed by making vertical cuts through the threads and prying the side of the lid to separate the threads of the lid from the body (Figure 4-4 A, B).
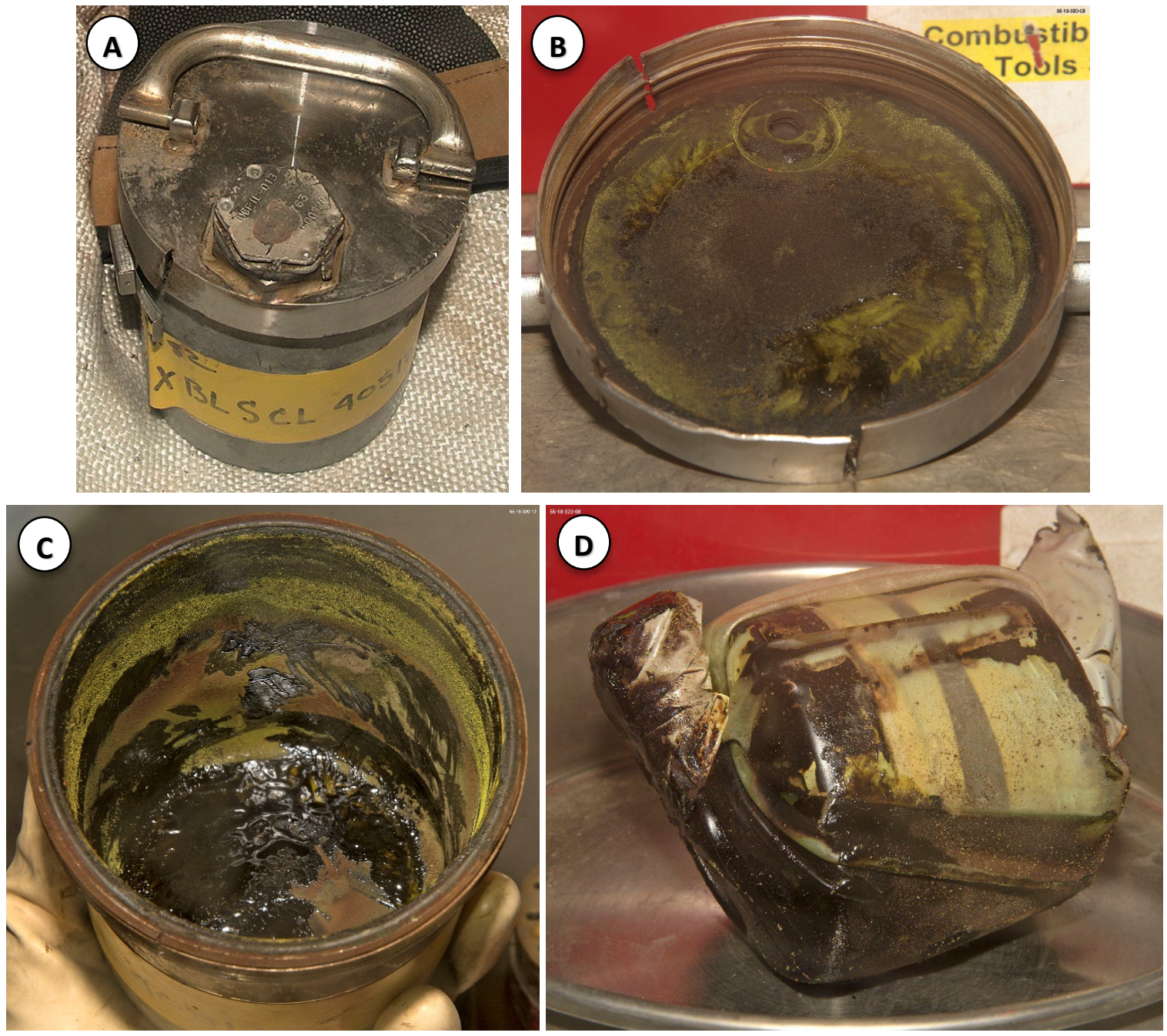

Figure 4-4. Photographs of $18 \mathrm{H} 7$ at unpacking. (A) External surfaces of container $18 \mathrm{H} 7$ showing white powder or etching on filter cap. (B) Inside surface of the lid covered in bag-out bag residue. Corrosion on threads shows gases escaped sealing surface. (C) Bag-out bag residue covering inside surface of the body. (D) Condition of bag-out bag.

Photographs were taken of the Hagan in lieu of the formal visual inspection. The external surface of the container had an etched appearance in some areas, such as the top of the 
filter, and corrosion was observed under the filter cap (Figure 4-4 A). The inside surfaces of the container were completely covered by gummy residue ranging in color from green to brown (Figure 4 B, C), and the entire bag surface had a sticky feel and color ranging from white to black (Figure 4D). Corrosion was observed on exterior of the filter and on the threads of both the lid and the body (Figure 4-5A,B).
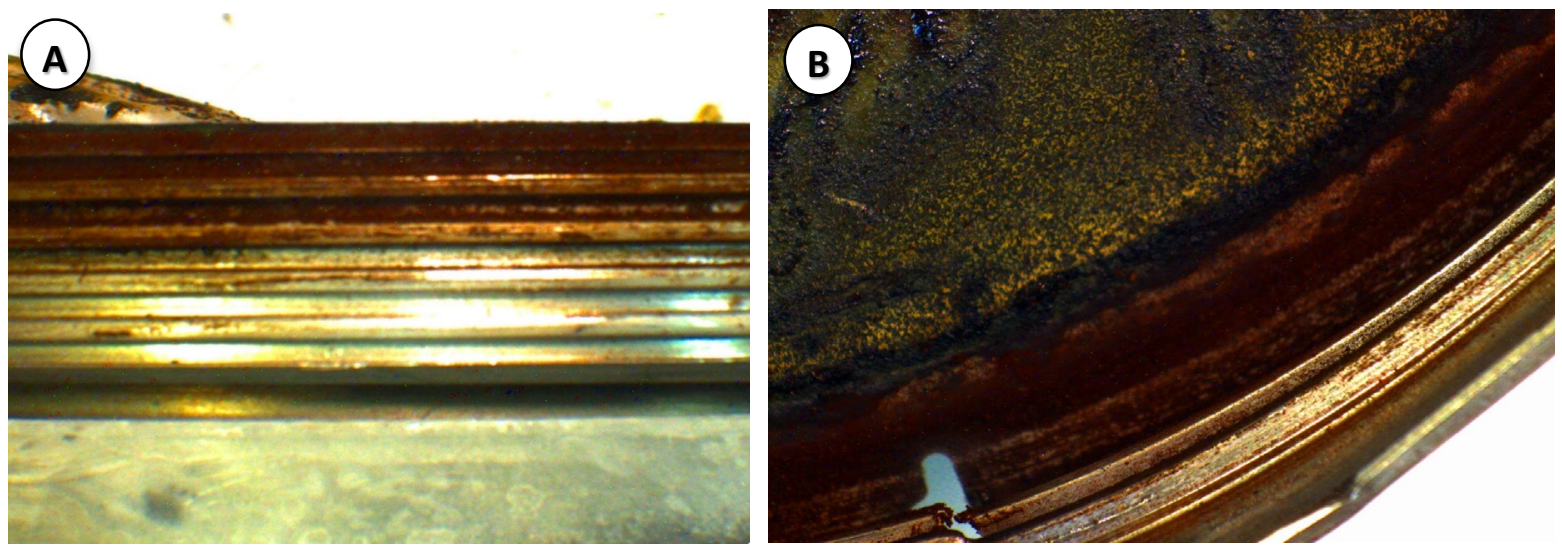

Figure 4-5. Images of $18 \mathrm{H} 7$ showing corrosion on threads.

The container lid and body were cleaned with $2 \mathrm{M}$ nitric acid and rinsed with deionized water. The container parts were photographed and then sectioned for further examination with optical microscopy. Photographs obtained of the cleaned container parts show large areas where general corrosion and pitting eroded the inside surfaces of the lid and body. The eroded areas appear as light spots on the following photographs. These light spots appear as large, agglomerated pits in the magnified images (Figure 4-7).

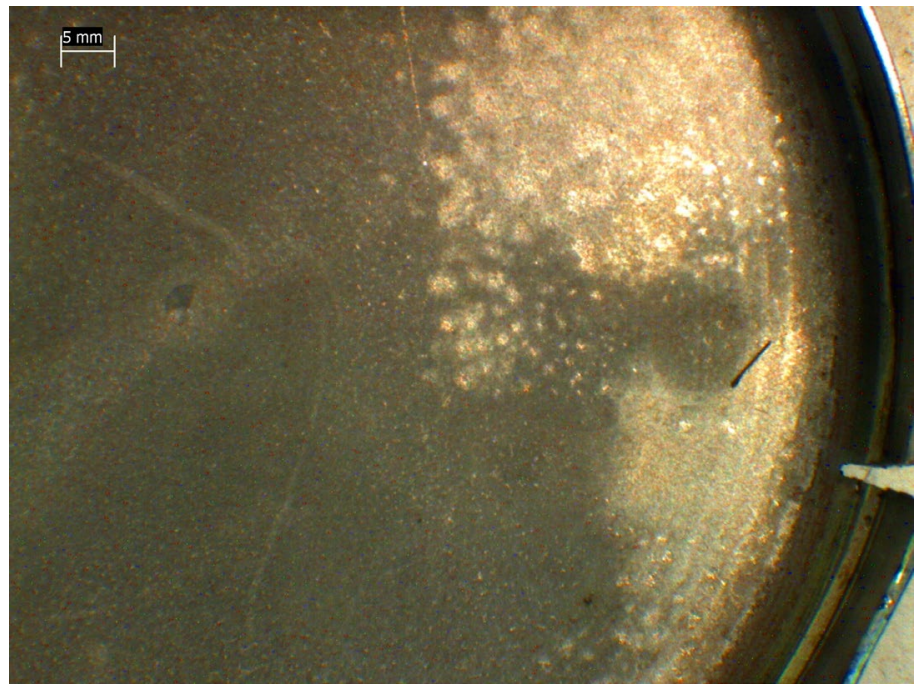

Figure 4-6. Eroded/pitted areas (showing as light spots) on the 18H7 Hagan lid. 

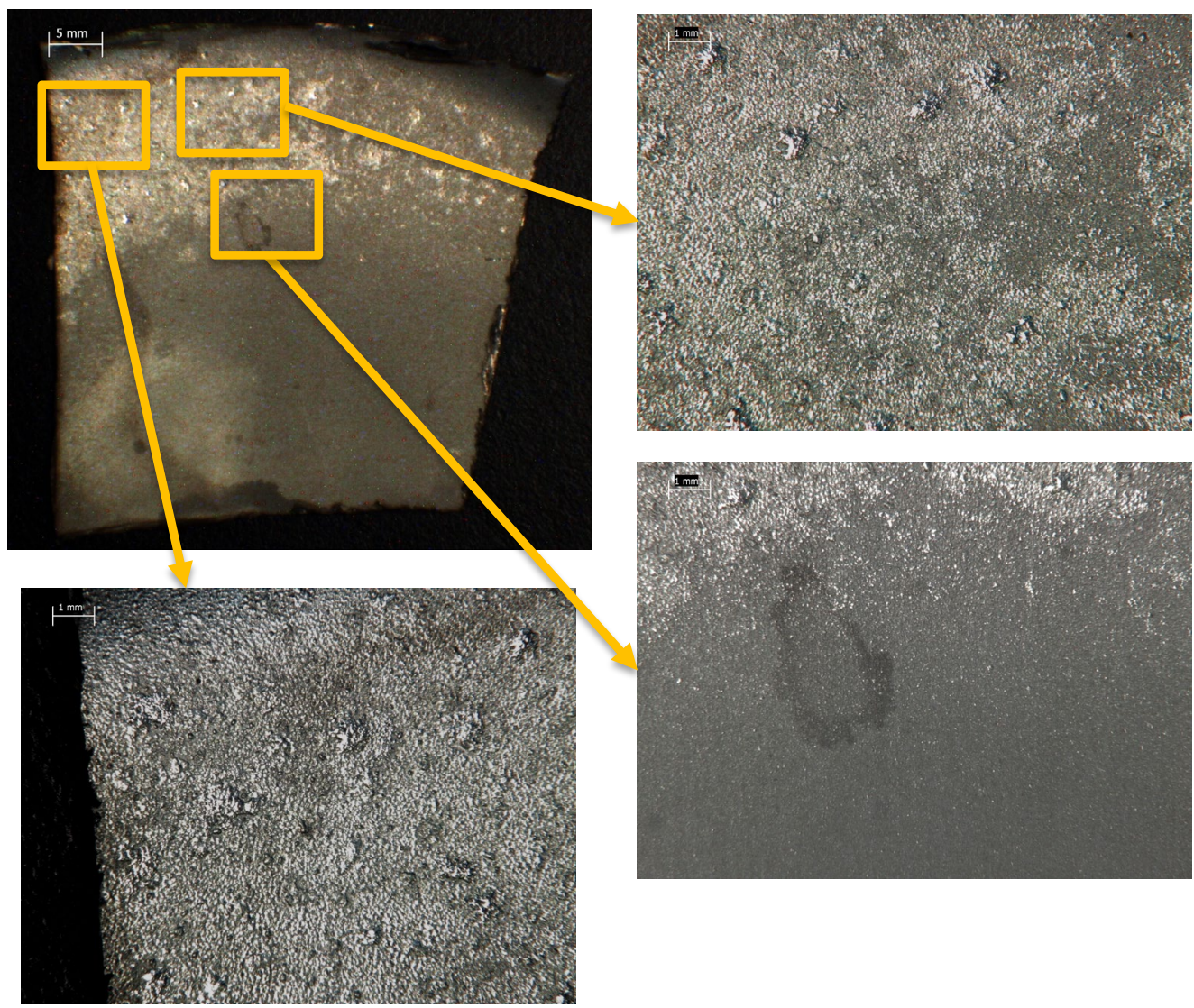

Figure 4-7. Magnified images of the eroded areas on container $18 \mathrm{H} 7$ bottom.

Optical microscopy showed pitting on the container lid and upper portion of the sidewall and general corrosion and erosion of the surface further down the wall and on the container bottom. Measurements of pit diameter and estimates of pit depth were obtained in areas where the native surface could be identified. Individual pits had diameters of approximately 100-150 microns and estimated depths of 20 to 40 microns (Figure 4-8.). Many of the pits were agglomerated. Farther down the wall of the container, large areas of surface erosion were observed. The extent of the surface erosion could not be determined because the native surface could not be identified. Nothing significant was found in the examination of the circumferential weld in the areas of the tack welds used to hold the bracket on the outside of the container. There were no cracks observed on the interior or exterior surfaces of the $18 \mathrm{H} 7$ container. 

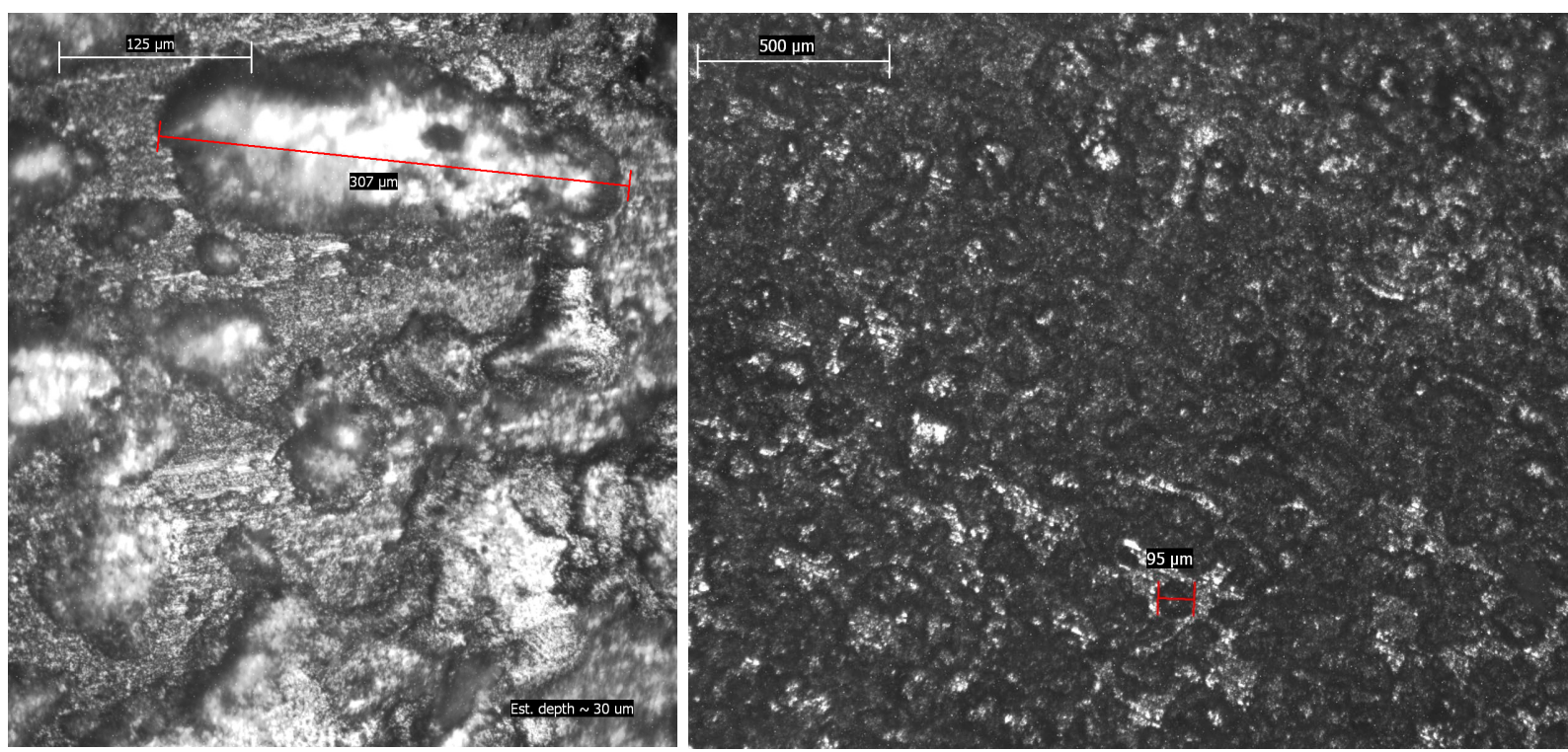

Figure 4-8. Pits measured by optical microscopy on the $18 \mathrm{H} 7$ Hagan container lid.

\subsubsection{Hagan Container, Surveillance, Sample \#18H8, 10/10 LANL-204 10/10-03064 XBLSCL1210, MT52, 432 g Pu, R83, MSE Salt, 6.9 W, 6 years}

Container $18 \mathrm{H} 8$ was packaged with MT52 DOR Salt. The inside surfaces of the Hagan were contamination free. No corrosion was observed inside the Hagan container. The condition of the bag-out bag and inner container are not known. The O-ring was inspected, and no issues were identified.

Container $18 \mathrm{H} 8$ had the filter particle penetration, filter pressure drop, O-ring durometer, and the helium leak tests performed. No issues were identified with the filter penetration test. However, the container failed the helium leak test because of a gross leak, and the O-ring failed the durometer test with an average measurement of 80.1 Shore M. The most likely cause of the failing leak test was due to the O-ring. 
Table 12. Unpacking data for Sample $18 \mathrm{H} 8$

\begin{tabular}{|c|c|}
\hline Surveillance sample number & $18 \mathrm{H} 8$ \\
\hline Operator & Unknown \\
\hline Date & $6 / 29 / 18$ \\
\hline Contamination found outside Hagan & No \\
\hline Contamination found inside Hagan & No \\
\hline Overall package weight & Not meas. \\
\hline Body serial & $10 / 10-03064$ \\
\hline Lid serial & 0-10 LANL-204 \\
\hline LOT ID & XBLSCL1210 \\
\hline Lead internal shield & Unknown \\
\hline Pewter outer shield & Unknown \\
\hline Outer container condition & Good \\
\hline O-ring installed & Yes \\
\hline Bag-out bag present & Yes \\
\hline Failed bag-out bag & No \\
\hline Liquid observed & No \\
\hline Condition of old bag-out bag & Unknown \\
\hline Type of inner & Unknown \\
\hline Condition of inner & Unknown \\
\hline Failed metal inner & No \\
\hline Bag-out bag replace & Unknown \\
\hline Inner replace & Unknown \\
\hline Beta/gamma dose (at lid/collar of Hagan) & Not meas. \\
\hline Beta/gamma dose (at $30 \mathrm{~cm}$ ) & Not meas. \\
\hline Neutron dose & Not meas. \\
\hline Comments & None \\
\hline
\end{tabular}

\subsubsection{O-Ring Tests}

\subsubsection{Visual Inspection of the O-rings}

Visual inspections were performed on the O-rings for containers $18 \mathrm{H} 6$ and $18 \mathrm{H} 8$. No issues were identified in the inspection.

\subsubsection{Leak Rate Tests}

The leak rate results for the Hagan storage containers are shown in Figure 9 for all Hagan containers measured in FY16 through FY19. The measured leak rate are shown in atm-cc/s of helium at 75 Torr into vacuum. One O-ring failed the leak test each in FY17 and FY18, with a measurement above the failure criterion of $1 \times 10^{-5} \mathrm{~atm} \mathrm{cc} / \mathrm{s}$, possibly resulting from the O-ring hardness being out of specification. 


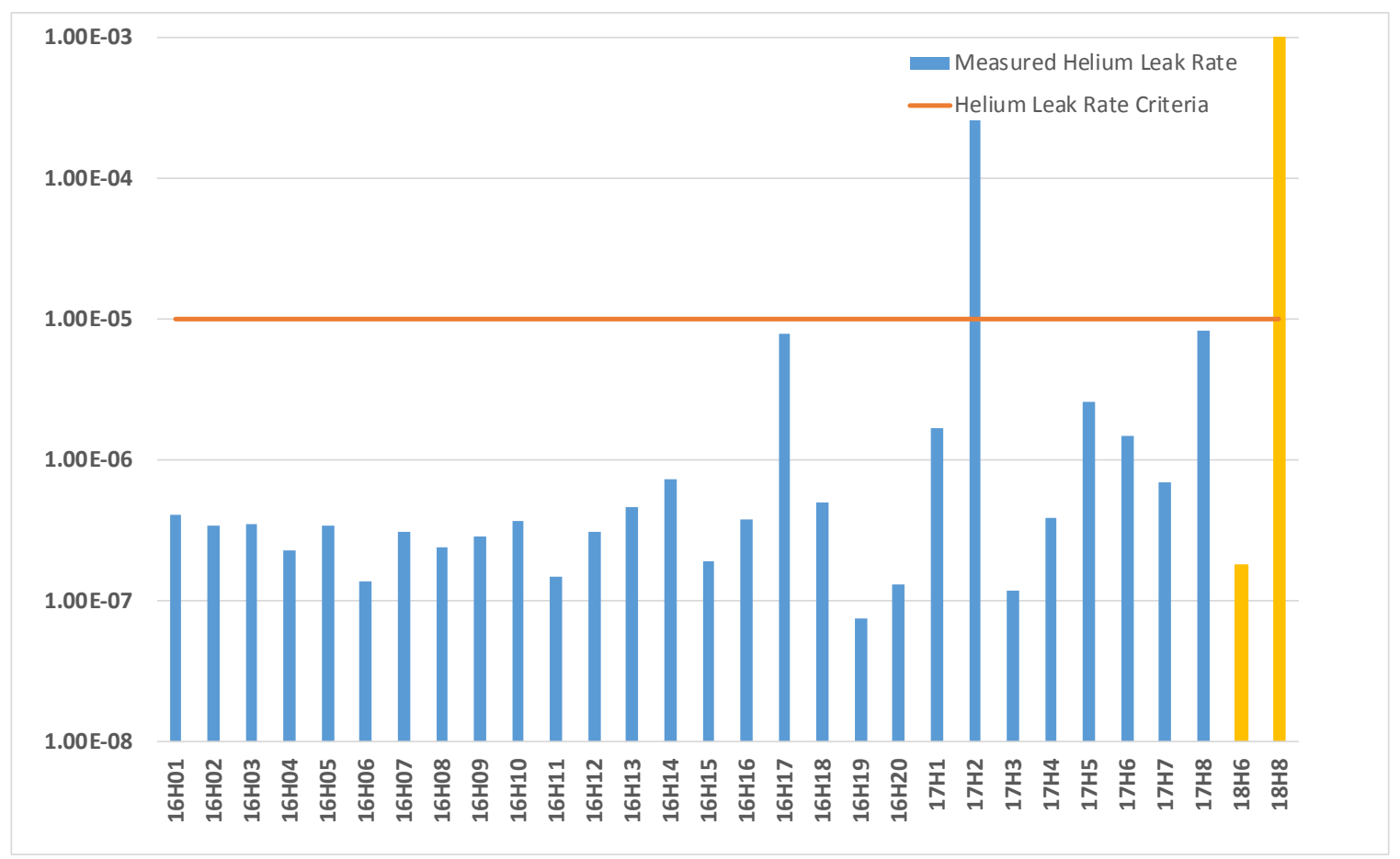

Figure 4-9. Leak rates measured for each Hagan container in FY16 (blue bars) FY17 (blue bars), and FY18 (orange bars). The red line shows the failure criteria. One container failed each in FY17 and FY18. Values on yaxis are in atmcc/sec.

\subsubsection{O-ring Hardness Tests}

The two Hagan O-rings tested in FY18 had hardness values of 79.9 and 80.1 durometer units, respectively. The O-ring from container $18 \mathrm{H} 8$ exceeded the specification limit of 80.0 durometer units. The FY18 hardness measurements are shown in Figure 10 as orange bars and compared with the FY16 and FY17 results (blue bars). The O-ring hardness for FY16 through FY18 containers ranged from 75.2 to 81.8 and had an average of 79.2 durometer units. The population of 20 O-rings used as a baseline in the lifetime extension studies have a hardness of $76.8 \pm 6.07$ durometer units, with a maximum of 78.6 and a minimum of 73.9 durometer units. These baseline O-ring measurements were performed on unused Hagan O-rings that had been stored in plastic bags. 


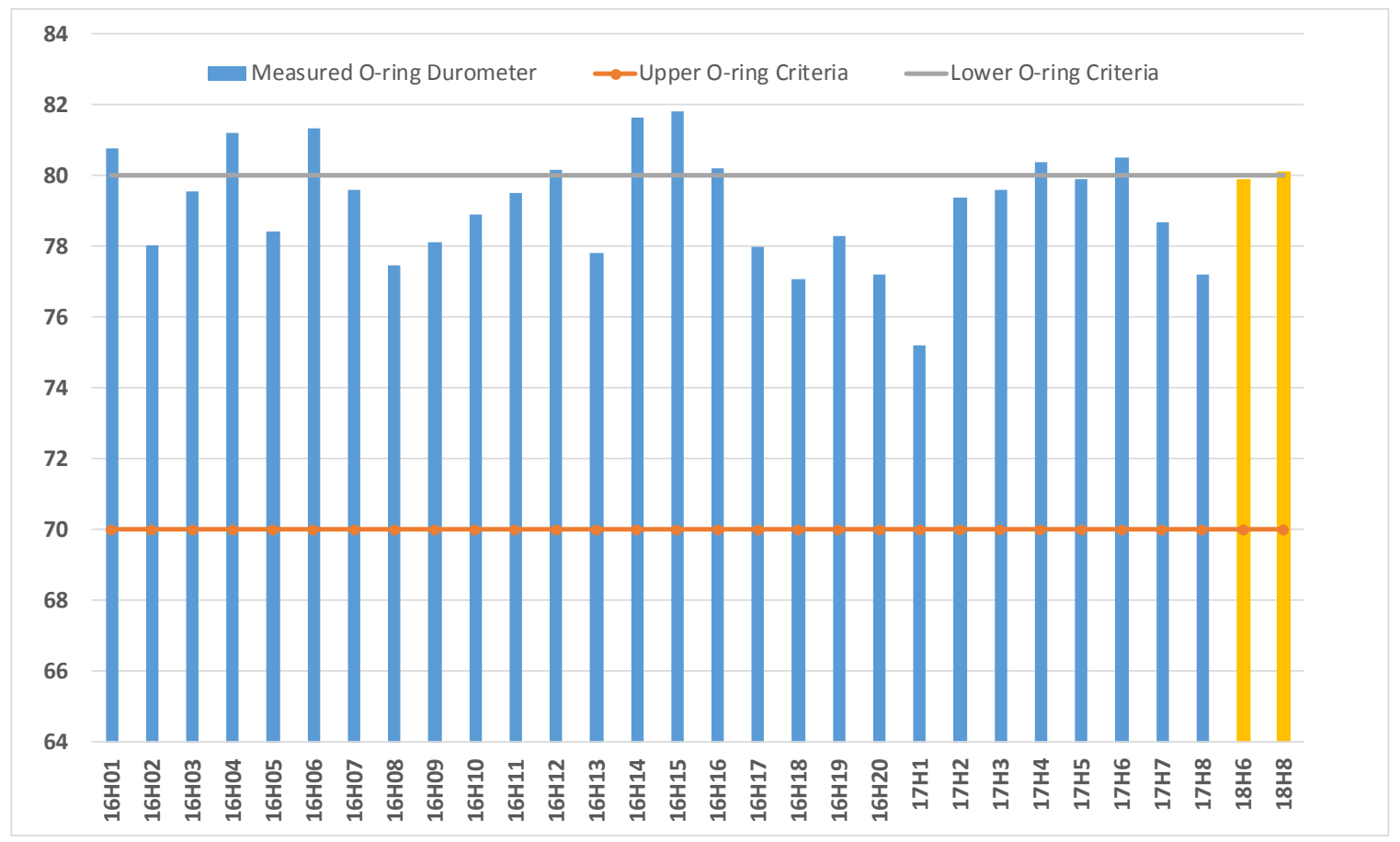

Figure 4-10. Durometer measurements for each Hagan container in FY16 (blue bars) FY17 (blue bars), and FY18 (orange bars). The red and purple lines represent the lower and upper bounds for the hardness, respectively. The O-ring durometer values on the $y$-axis are Shore-M hardness values.

\subsubsection{Filter Tests}

\subsubsection{Particle Penetration}

The aerosol data are reported as a percent penetration, also known as the percent leakage. Two Hagan containers were tested in FY18 and compared with the results from FY16 and FY17 in Figure 11. The set of particle penetration measurements obtained in FY16 through FY18 is narrowly distributed and is at least a factor of 2 lower than the requirement. No filters have failed the particle penetration test. 
0.035

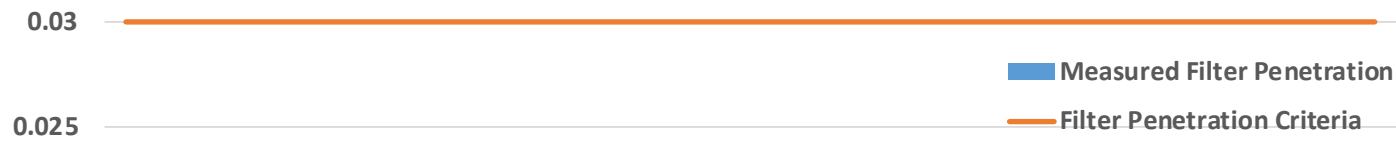

0.02

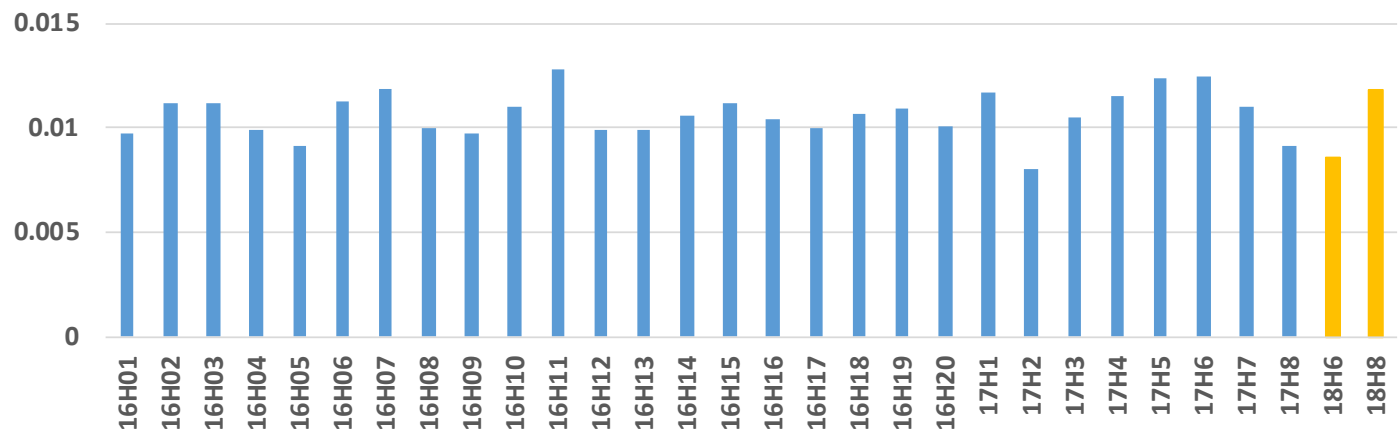

Figure 4-11. Filter particle penetration measurements for each Hagan container measured in FY16 (blue bars) FY17 (blue bars), and FY18 (orange bars). The red line represents the upper bound for filter penetration. Values on $y$-axis are the filter efficiency.

\subsubsection{Pressure Drop}

Two Hagan containers were tested in FY18 and compared with the results from FY16 and FY17 in Figure 12. The FY18 pressure drop measurements for containers $18 \mathrm{H} 6$ and $18 \mathrm{H} 8$ are 0.868 and 0.797 in. W.C., respectively. The average for FY16 through FY18 is 0.75 in. W.C. 


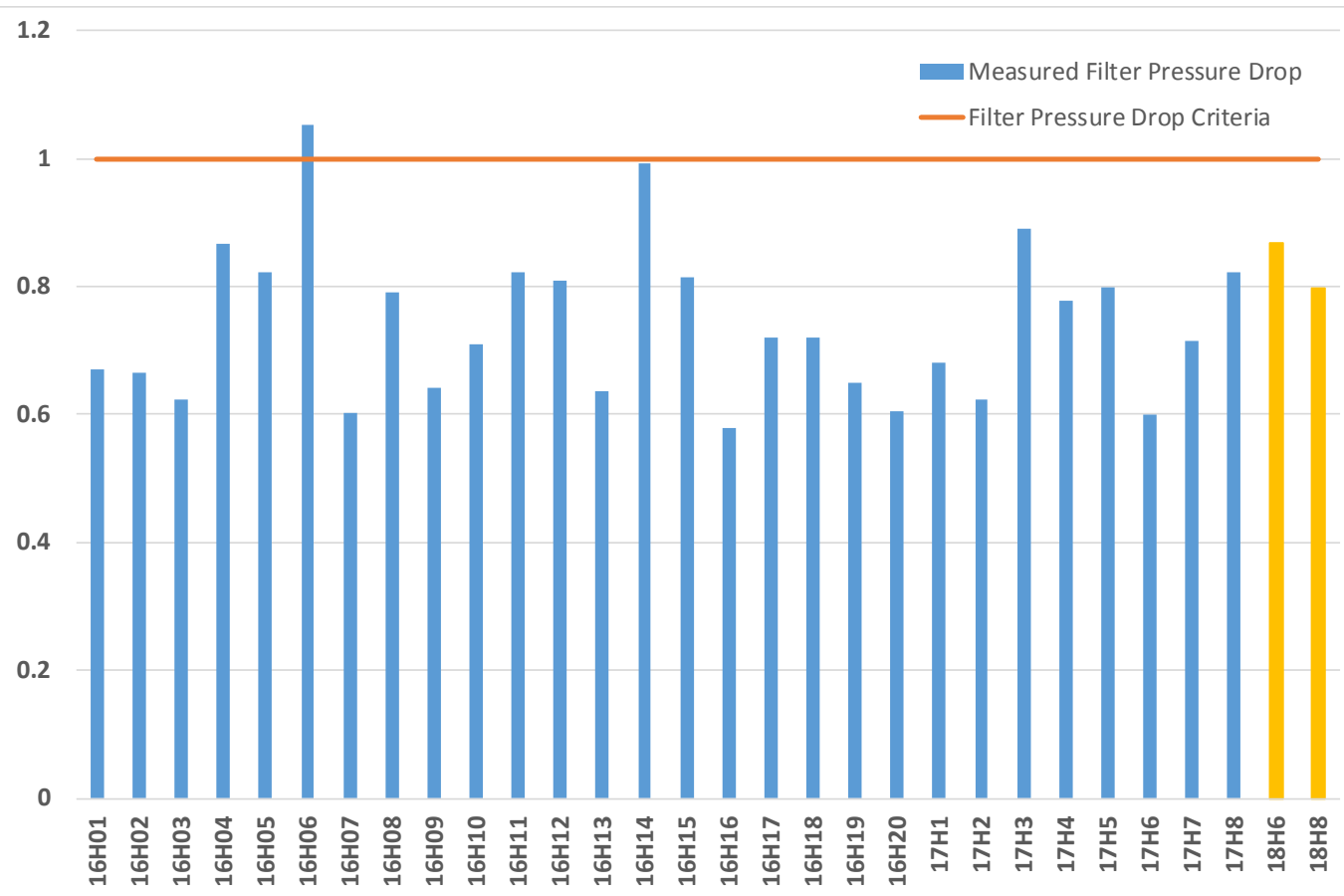

Figure 4-12. Filter pressure drop measurements for each Hagan container measured in FY16 (blue bars) FY17 (blue bars), and FY18 (yellow bars). The red line represents the upper bound for filter penetration. Values on y-axis are pressure drop in units of inches W.C.

\subsubsection{Corrosion}

Corrosion has been found on 4 of 8 Hagan containers targeted for surveillance this year. The amount of the corrosion in 3 of the Hagan containers was minor in appearance and seemed to be in close proximity to the PVC bag or tape. The corrosion on Hagan container $18 \mathrm{H} 7$ was the worst observed in any container to date. This container had large, agglomerated corrosion pits that are hundreds of microns in diameter and up to 40 microns deep. The corrosion in this container was observed beyond the O-ring seal resulting in the lid being stuck in place on the container. This 1-quart container was packaged with an MSE salt (R83). The major factor resulting in the extensive corrosion was the $7 \mathrm{~W}$ irradiating a PVC bag-out bag in a small volume for 13 years. The conditions inside this container supported corrosion at the time of opening, which was evident from the corrosion inside the SAVY 4000 container used to overpack the container for 14 months.

The container management team formed a corrosion working group, bringing together people from the 3013 studies and SAVY 4000/Hagan surveillance team members to further investigate the corrosion effects and guide the container surveillance. Surveillance in FY19 will continue to target containers with a high likelihood for corrosion. These containers include other containers with MSE salts with a high wattage to volume ratio or bag degradation factor [7]. Additionally, the future surveillance will target other material forms and material types not yet included in the surveillance program to investigate corrosion in these containers. 


\subsection{SAVY 4000}

The results for the SAVY 4000 storage containers are summarized in Table 13. The results for the SAVY 4000 transfer containers are summarized in Table 14. All O-rings passed a helium leak test and durometer measurements. All filters passed the criteria for efficiency and pressure drop. The exception is containers 18S6 and 18S7, both of which had to be introduced so data collected was derived from photographic evidence only. All SAVY 4000 storage containers with the exception of those introduced were returned to service with the same item packaged inside. 
Table 13. Surveillance test results for SAVY 4000 storage containers

\begin{tabular}{|c|c|c|c|c|c|c|c|c|c|c|c|}
\hline ID & $\begin{array}{l}\text { Container } \\
\text { Serial No. } \\
\text { and Size }\end{array}$ & $\begin{array}{l}\text { Container } \\
\text { Visual } \\
\text { Inspection }\end{array}$ & $\begin{array}{c}\text { Corrosion } \\
\text { Ranking }\end{array}$ & $\begin{array}{c}\text { O-Ring } \\
\text { Visual } \\
\text { Inspection }\end{array}$ & $\begin{array}{c}\text { Material Name } \\
\text { and } \\
\text { IDC }\end{array}$ & $\begin{array}{c}\text { Sample } \\
\text { Power } \\
\text { (W) }\end{array}$ & $\begin{array}{c}\text { Package } \\
\text { Age } \\
\text { (y) }\end{array}$ & $\begin{array}{c}\text { Filter } \\
\text { Particle } \\
\text { Penetration } \\
\text { (\%) } \\
\pm \mathbf{0 . 0 0 0 2}\end{array}$ & $\begin{array}{l}\text { Filter } \\
\text { Pressure } \\
\text { Drop } \\
\text { (in W.C) } \\
\pm 0.02\end{array}$ & $\begin{array}{l}\text { Helium } \\
\text { Leak } \\
\text { Rate } \\
\left(\frac{a t m-c c}{s}\right)\end{array}$ & $\begin{array}{c}\text { O-ring } \\
\text { Durometer } \\
\text { (Shore M) } \\
\pm 2.15\end{array}$ \\
\hline $18 S 1$ & $\begin{array}{c}111308080 \\
8 \text { Quart }\end{array}$ & $\begin{array}{l}\text { Corrosion } \\
\text { around the } \\
\text { weld inside } \\
\text { the container } \\
\text { and residue } \\
\text { around the } \\
\text { inside the of } \\
\text { the container }\end{array}$ & 2 & $\begin{array}{c}\text { No } \\
\text { comments }\end{array}$ & $\begin{array}{c}\text { BLO-39-11-16 } \\
\text { C21 }\end{array}$ & 5 & 2.4 & $0.0002 \%$ & 0.625 & $\begin{array}{c}2.10 \mathrm{E}- \\
08\end{array}$ & 57.3 \\
\hline $18 S 2$ & $\begin{array}{c}031105052 \\
5 \text { Quart }\end{array}$ & $\begin{array}{l}\text { Thumb latch is } \\
\text { a little sticky } \\
\text { but still works }\end{array}$ & 0 & $\begin{array}{c}\text { No } \\
\text { comments }\end{array}$ & $\begin{array}{c}\text { CXLOX082911 } \\
\text { C21 }\end{array}$ & 2.07 & 6.1 & $0.0001 \%$ & 0.508 & $\begin{array}{c}3.10 \mathrm{E}- \\
08\end{array}$ & 57.5 \\
\hline $18 S 3 b$ & $\begin{array}{c}111308059 \\
8 \text { Quart }\end{array}$ & $\begin{array}{l}\text { Discoloration } \\
\text { on the inner } \\
\text { side of the } \\
\text { locking ring. }\end{array}$ & 0 & $\begin{array}{c}\text { No } \\
\text { comments }\end{array}$ & $\begin{array}{l}\text { XAP6 (outer) } \\
\text { M44 }\end{array}$ & 0.21 & 2.0 & $0.0000 \%$ & 0.682 & $\begin{array}{c}3.80 \mathrm{E}- \\
08\end{array}$ & 57.5 \\
\hline
\end{tabular}




\begin{tabular}{|c|c|c|c|c|c|c|c|c|c|c|c|}
\hline ID & $\begin{array}{c}\text { Container } \\
\text { Serial No. } \\
\text { and Size }\end{array}$ & $\begin{array}{l}\text { Container } \\
\text { Visual } \\
\text { Inspection }\end{array}$ & $\begin{array}{c}\text { Corrosion } \\
\text { Ranking }\end{array}$ & $\begin{array}{c}\text { O-Ring } \\
\text { Visual } \\
\text { Inspection }\end{array}$ & $\begin{array}{c}\text { Material Name } \\
\text { and } \\
\text { IDC }\end{array}$ & $\begin{array}{c}\text { Sample } \\
\text { Power } \\
\text { (W) }\end{array}$ & $\begin{array}{c}\text { Package } \\
\text { Age } \\
\text { (y) }\end{array}$ & $\begin{array}{c}\text { Filter } \\
\text { Particle } \\
\text { Penetration } \\
\text { (\%) } \\
\pm \mathbf{0 . 0 0 0 2}\end{array}$ & $\begin{array}{c}\text { Filter } \\
\text { Pressure } \\
\text { Drop } \\
\text { (in W.C) } \\
\pm 0.02\end{array}$ & $\begin{array}{l}\text { Helium } \\
\text { Leak } \\
\text { Rate } \\
\left(\frac{a t m-c c}{s}\right)\end{array}$ & $\begin{array}{c}\text { O-ring } \\
\text { Durometer } \\
\text { (Shore M) } \\
\pm 2.15\end{array}$ \\
\hline $18 S 4$ & $\begin{array}{l}031105051 \\
5 \text { Quart }\end{array}$ & $\begin{array}{l}\text { Sticky thumb } \\
\text { latch. } \\
\text { Brown coating } \\
\text { on inside } \\
\text { surfaces. Small } \\
\text { dimple on } \\
\text { bottom of the } \\
\text { container }\end{array}$ & 1 & $\begin{array}{c}\text { No } \\
\text { comments }\end{array}$ & $\begin{array}{c}\text { ROTRBJ-1C1 } \\
\text { R26 }\end{array}$ & 1.19 & 6.6 & $0.0002 \%$ & 0.555 & $\begin{array}{c}3.30 \mathrm{E}- \\
08\end{array}$ & 57.8 \\
\hline $18 S 5$ & $\begin{array}{c}121103083 \\
3 \text { Quart }\end{array}$ & $\begin{array}{l}\text { Isolated spots } \\
\text { of corrosion } \\
\text { on inside } \\
\text { surfaces. } \\
\text { Anodizing } \\
\text { color on the } \\
\text { locking ring } \\
\text { has changed. } \\
\text { Scratch on } \\
\text { locking ring. }\end{array}$ & 1 & $\begin{array}{c}\text { No } \\
\text { comments }\end{array}$ & $\begin{array}{c}\text { XBLSCL1217 } \\
\text { R83 }\end{array}$ & 2.85 & 4.9 & $0.0001 \%$ & 0.658 & $\begin{array}{c}1.20 \mathrm{E}- \\
08\end{array}$ & 57.6 \\
\hline
\end{tabular}




\begin{tabular}{|c|c|c|c|c|c|c|c|c|c|c|c|}
\hline ID & $\begin{array}{l}\text { Container } \\
\text { Serial No. } \\
\text { and Size }\end{array}$ & $\begin{array}{c}\text { Container } \\
\text { Visual } \\
\text { Inspection }\end{array}$ & $\begin{array}{c}\text { Corrosion } \\
\text { Ranking }\end{array}$ & $\begin{array}{c}\text { O-Ring } \\
\text { Visual } \\
\text { Inspection }\end{array}$ & $\begin{array}{c}\text { Material Name } \\
\text { and } \\
\text { IDC }\end{array}$ & $\begin{array}{c}\text { Sample } \\
\text { Power } \\
\text { (W) }\end{array}$ & $\begin{array}{c}\text { Package } \\
\text { Age } \\
\text { (y) }\end{array}$ & $\begin{array}{c}\text { Filter } \\
\text { Particle } \\
\text { Penetration } \\
\mathbf{( \% )} \\
\pm \mathbf{0 . 0 0 0 2}\end{array}$ & $\begin{array}{c}\text { Filter } \\
\text { Pressure } \\
\text { Drop } \\
\text { (in W.C) } \\
\pm 0.02\end{array}$ & $\begin{array}{l}\text { Helium } \\
\text { Leak } \\
\text { Rate } \\
\left(\frac{a t m-c c}{s}\right)\end{array}$ & $\begin{array}{c}\text { O-ring } \\
\text { Durometer } \\
\text { (Shore M) } \\
\pm 2.15\end{array}$ \\
\hline $18 S 6$ & $\begin{array}{c}121103062 \\
3 \text { Quart }\end{array}$ & $\begin{array}{l}\text { Contamination } \\
\text { found on } \\
\text { inside of } \\
\text { container }\end{array}$ & 1 & $\begin{array}{c}\text { Not } \\
\text { performed }\end{array}$ & $\begin{array}{c}\text { SLTF1323A } \\
\text { R42 }\end{array}$ & 5 & 5.4 & NM & NM & NM & NM \\
\hline $18 S 7$ & $\begin{array}{c}091205130 \\
5 \text { Quart }\end{array}$ & $\begin{array}{l}\text { White dry } \\
\text { residue found } \\
\text { outside of } \\
\text { container near } \\
\text { filter. } \\
\text { Corrosion was } \\
\text { found on the } \\
\text { inside of the } \\
\text { container near } \\
\text { the weld. } \\
\text { Heavy } \\
\text { amounts of } \\
\text { bag-out bag } \\
\text { liquid residue } \\
\text { was found on } \\
\text { the inside } \\
\text { bottom of the } \\
\text { container. }\end{array}$ & 1 & $\begin{array}{c}\text { Not } \\
\text { performed }\end{array}$ & $\begin{array}{c}091205130 \\
\text { (AQR162NP=max } \\
\text { g item) } \\
\text { C21 }\end{array}$ & 16.6 & 3.8 & NM & NM & NM & NM \\
\hline
\end{tabular}


Table 14. Surveillance test results for SAVY 4000 transfer containers.

\begin{tabular}{|c|c|c|c|c|c|c|c|}
\hline ID & $\begin{array}{l}\text { Container } \\
\text { Serial No. } \\
\text { and Size }\end{array}$ & Container Visual Inspection & $\begin{array}{l}\text { O-Ring Visual } \\
\text { Inspection }\end{array}$ & $\begin{array}{c}\text { Filter Particle } \\
\text { Penetration } \\
\text { (\%) } \\
\pm \mathbf{0 . 0 0 0 2}\end{array}$ & $\begin{array}{l}\text { Filter } \\
\text { Pressure } \\
\text { Drop } \\
\text { (in W.C) } \\
\pm 0.02\end{array}$ & $\begin{array}{c}\text { Helium } \\
\text { Leak Rate } \\
\left(\frac{a t m-c c}{s}\right)\end{array}$ & $\begin{array}{c}\text { O-ring } \\
\text { Durometer } \\
\text { (Shore M) } \pm \\
2.15\end{array}$ \\
\hline $1 \mathrm{~T}$ & $\begin{array}{c}\text { 011701145L/B } \\
1 \text { Quart }\end{array}$ & No issues reported & $\begin{array}{l}\text { O-ring had some } \\
\text { dust on but was } \\
\text { easily cleaned, o- } \\
\text { ring passes visual }\end{array}$ & 0.0005 & 0.607 & $1.60 \mathrm{E}-08$ & 57.3 \\
\hline $2 T$ & $\begin{array}{c}\text { 031403046L/B } \\
3 \text { Quart }\end{array}$ & $\begin{array}{l}\text { Tape residue on the outside of the lid } \\
\text { but the overall integrity passes }\end{array}$ & No issues reported & 0.0003 & 0.706 & $4.0 \mathrm{E}-08$ & 56.8 \\
\hline $3 T$ & $\begin{array}{c}\text { 031403054L/B } \\
3 \text { Quart }\end{array}$ & $\begin{array}{l}\text { Tape residue on the outside of the lid. } \\
\text { Container still passes }\end{array}$ & $\begin{array}{l}\text { There as small } \\
\text { inclusions in the } \\
\text { surface from } \\
\text { manufacturer but } \\
\text { won't compromise } \\
\text { the sealing } \\
\text { properties, o-ring } \\
\text { still pass }\end{array}$ & 0.0002 & 0.629 & $1.9 \mathrm{E}-08$ & 56.4 \\
\hline $4 \mathrm{~T}$ & $\begin{array}{c}\text { 031403160L/B } \\
3 \text { Quart }\end{array}$ & Very small dimple on bottom radius & No issues reported & 0.0001 & 0.659 & $1.6 \mathrm{E}-08$ & 56.7 \\
\hline
\end{tabular}




\begin{tabular}{|c|c|c|c|c|c|c|c|}
\hline ID & $\begin{array}{l}\text { Container } \\
\text { Serial No. } \\
\text { and Size }\end{array}$ & Container Visual Inspection & $\begin{array}{l}\text { O-Ring Visual } \\
\text { Inspection }\end{array}$ & $\begin{array}{c}\text { Filter Particle } \\
\text { Penetration } \\
(\%) \\
\pm 0.0002\end{array}$ & $\begin{array}{c}\text { Filter } \\
\text { Pressure } \\
\text { Drop } \\
\text { (in W.C) } \\
\pm 0.02\end{array}$ & $\begin{array}{l}\text { Helium } \\
\text { Leak Rate } \\
\left(\frac{a t m-c c}{s}\right)\end{array}$ & $\begin{array}{c}\text { O-ring } \\
\text { Durometer } \\
\text { (Shore M) } \pm \\
2.15\end{array}$ \\
\hline $5 T$ & $\begin{array}{c}\text { 031403088L/B } \\
3 \text { Quart }\end{array}$ & $\begin{array}{l}\text { There is a crease on the side of the } \\
\text { container that has been previously } \\
\text { reviewed, signed and deemed O.K. } \\
\text { There is another small dent on the } \\
\text { bottom side of the container but it } \\
\text { does not compromise the containers } \\
\text { ability to balance on flat surfaces or } \\
\text { compromises the overall structure. } \\
\text { Pass }\end{array}$ & No issues reported & 0.0003 & 0.658 & $1.7 \mathrm{E}-08$ & 57.4 \\
\hline $6 \mathrm{~T}$ & $\begin{array}{c}\text { 071201136L/B } \\
1 \text { Quart }\end{array}$ & $\begin{array}{l}\text { There is a small line of residue on the } \\
\text { inner side body. Container still passes }\end{array}$ & No issues reported & 0.0002 & 0.661 & $3.1 \mathrm{E}-08$ & 58.4 \\
\hline 7T & $\begin{array}{c}\text { 071203168B } \\
\text { 071203190L } \\
3 \text { Quart }\end{array}$ & $\begin{array}{l}\text { There is radial scratch on top of the lid, } \\
\text { Container still passes }\end{array}$ & No issues reported & 0.0002 & 0.681 & $2.1 \mathrm{E}-08$ & 57.5 \\
\hline $8 \mathrm{~T}$ & $\begin{array}{c}\text { 081301145L/B } \\
1 \text { Quart }\end{array}$ & $\begin{array}{l}\text { Small amount of dried brown residue } \\
\text { on bottom radius, outside of container } \\
\text { it was easily removed. }\end{array}$ & No issues reported & 0.0003 & 0.652 & $2.9 \mathrm{E}-08$ & 56.2 \\
\hline
\end{tabular}




\begin{tabular}{|c|c|c|c|c|c|c|c|}
\hline ID & $\begin{array}{l}\text { Container } \\
\text { Serial No. } \\
\text { and Size }\end{array}$ & Container Visual Inspection & $\begin{array}{l}\text { O-Ring Visual } \\
\text { Inspection }\end{array}$ & $\begin{array}{c}\text { Filter Particle } \\
\text { Penetration } \\
\text { (\%) } \\
\pm \mathbf{0 . 0 0 0 2}\end{array}$ & $\begin{array}{l}\text { Filter } \\
\text { Pressure } \\
\text { Drop } \\
\text { (in W.C) } \\
\pm 0.02\end{array}$ & $\begin{array}{l}\text { Helium } \\
\text { Leak Rate } \\
\left(\frac{a t m-c c}{s}\right)\end{array}$ & $\begin{array}{c}\text { O-ring } \\
\text { Durometer } \\
\text { (Shore M) } \pm \\
2.15\end{array}$ \\
\hline 9T & $\begin{array}{c}081305013 \mathrm{~L} / \mathrm{B} \\
5 \text { Quart }\end{array}$ & $\begin{array}{l}\text { There is a lot of scratches on the } \\
\text { locking ring but overall the container } \\
\text { passes visual integrity }\end{array}$ & $\begin{array}{l}\text { There was some } \\
\text { dust but after } \\
\text { cleaning the o-ring } \\
\text { there were no } \\
\text { more defects or } \\
\text { debris. }\end{array}$ & 0.0002 & 0.647 & $3.5 \mathrm{E}-08$ & 57.1 \\
\hline 10T & $\begin{array}{l}\text { 081305044L/B } \\
5 \text { Quart }\end{array}$ & $\begin{array}{l}\text { There is evidence of liquid that has } \\
\text { been spilled or splashed onto the } \\
\text { interior walls, but the container } \\
\text { integrity still passes. }\end{array}$ & $\begin{array}{l}\text { There was dust on } \\
\text { the o-ring but after } \\
\text { cleaning the o-ring } \\
\text { there were no } \\
\text { other defects or } \\
\text { debris present. }\end{array}$ & 0.0003 & 0.637 & $3.6 \mathrm{E}-08$ & 56.8 \\
\hline $11 \mathrm{~T}$ & $\begin{array}{c}\text { 081305092L/B } \\
5 \text { Quart }\end{array}$ & $\begin{array}{l}\text { Small crease on bottom radius and a } \\
\text { few small dimples on bottom radius. } \\
\text { The thumb latch is a little sticky but } \\
\text { still is functional. Pass }\end{array}$ & No issues reported & 0.0005 & 0.621 & $2.4 \mathrm{E}-08$ & 56.7 \\
\hline $12 \mathrm{~T}$ & $\begin{array}{c}081305221 \mathrm{~L} / \mathrm{B} \\
5 \text { Quart }\end{array}$ & No issues reported & No issues reported & 0.0001 & 0.657 & $1.8 \mathrm{E}-08$ & 58.1 \\
\hline $13 T$ & $\begin{array}{c}111501011 \mathrm{~L} / \mathrm{B} \\
1 \text { Quart }\end{array}$ & No issues reported & $\begin{array}{l}\text { Dull in color but } \\
\text { still passes visual }\end{array}$ & 0.0000 & 0.762 & $3.4 \mathrm{E}-08$ & 57.5 \\
\hline
\end{tabular}




\begin{tabular}{|c|c|c|c|c|c|c|c|}
\hline ID & $\begin{array}{l}\text { Container } \\
\text { Serial No. } \\
\text { and Size }\end{array}$ & Container Visual Inspection & $\begin{array}{l}\text { O-Ring Visual } \\
\text { Inspection }\end{array}$ & $\begin{array}{c}\text { Filter Particle } \\
\text { Penetration } \\
\text { (\%) } \\
\pm \mathbf{0 . 0 0 0 2}\end{array}$ & $\begin{array}{c}\text { Filter } \\
\text { Pressure } \\
\text { Drop } \\
\text { (in W.C) } \\
\pm 0.02\end{array}$ & $\begin{array}{l}\text { Helium } \\
\text { Leak Rate } \\
\left(\frac{a t m-c c}{s}\right)\end{array}$ & $\begin{array}{c}\text { O-ring } \\
\text { Durometer } \\
\text { (Shore M) } \pm \\
2.15\end{array}$ \\
\hline 14T & $\begin{array}{c}\text { 111501014B } \\
011701068 \mathrm{~L} \\
1 \text { Quart }\end{array}$ & $\begin{array}{l}\text { Small amounts of residue on inner side } \\
\text { wall. Container still passes }\end{array}$ & No issues reported & 0.0000 & 0.699 & $2.6 \mathrm{E}-08$ & 57.9 \\
\hline 15T & $\begin{array}{c}111501076 \mathrm{~L} / \mathrm{B} \\
1 \text { Quart }\end{array}$ & No issues reported & $\begin{array}{l}\text { O-ring had dust but } \\
\text { was removed by } \\
\text { cleaning, o-ring } \\
\text { passes visual }\end{array}$ & 0.0001 & 0.754 & $1.8 \mathrm{E}-08$ & 57.4 \\
\hline $16 \mathrm{~T}$ & $\begin{array}{c}\text { 031403066L/B } \\
3 \text { Quart }\end{array}$ & No issues reported & No issues reported & 0.0004 & 0.601 & $2.0 \mathrm{E}-08$ & 56.9 \\
\hline 17T & $\begin{array}{c}031403087 \mathrm{~L} / \mathrm{B} \\
3 \text { Quart }\end{array}$ & No issues reported & No issues reported & 0.0002 & 0.625 & $1.3 \mathrm{E}-08$ & 56.9 \\
\hline $18 \mathrm{~T}$ & $\begin{array}{c}\text { 031403180L/B } \\
3 \text { Quart }\end{array}$ & No issues reported & No issues reported & 0.0003 & 0.675 & $1.7 \mathrm{E}-08$ & 56.7 \\
\hline 19T & $\begin{array}{c}\text { 041212006L/B } \\
12 \text { Quart }\end{array}$ & No issues reported & No issues reported & 0.0001 & 0.619 & $9.5 \mathrm{E}-08$ & 58.6 \\
\hline
\end{tabular}




\begin{tabular}{|c|c|c|c|c|c|c|c|}
\hline ID & $\begin{array}{l}\text { Container } \\
\text { Serial No. } \\
\text { and Size }\end{array}$ & Container Visual Inspection & $\begin{array}{l}\text { O-Ring Visual } \\
\text { Inspection }\end{array}$ & $\begin{array}{c}\text { Filter Particle } \\
\text { Penetration } \\
\text { (\%) } \\
\pm \mathbf{0 . 0 0 0 2}\end{array}$ & $\begin{array}{c}\text { Filter } \\
\text { Pressure } \\
\text { Drop } \\
\text { (in W.C) } \\
\pm 0.02\end{array}$ & $\begin{array}{l}\text { Helium } \\
\text { Leak Rate } \\
\left(\frac{a t m-c c}{s}\right)\end{array}$ & $\begin{array}{c}\text { O-ring } \\
\text { Durometer } \\
\text { (Shore M) } \pm \\
2.15\end{array}$ \\
\hline 20T & $\begin{array}{c}\text { 071201072L/B } \\
1 \text { Quart }\end{array}$ & No issues reported & No issues reported & 0.0003 & 0.644 & $2.5 \mathrm{E}-08$ & 56.9 \\
\hline $21 \mathrm{~T}$ & $\begin{array}{c}081305047 \mathrm{~L} / \mathrm{B} \\
5 \text { Quart }\end{array}$ & $\begin{array}{l}\text { The anodization is flaking off the } \\
\text { locking ring. Continuer still passes }\end{array}$ & $\begin{array}{l}\text { Small black } \\
\text { inclusions from } \\
\text { manufacturing, o- } \\
\text { ring still passes }\end{array}$ & 0.0003 & 0.622 & $2.2 \mathrm{E}-08$ & 57.4 \\
\hline
\end{tabular}




\subsubsection{Visual Inspections}

Visual inspections of each container revealed SAVY 4000 containers with corrosion issues. Photographs of these containers were taken and the containers are held if further analysis is determined to be necessary.

\subsubsection{SAVY 4000 Container, Surveillance, Sample 18S1, SN 111308080, BLO-39-11- 16, MT57, 544g, C21, Compound; Dioxide, 5 W, 2.4 years}

Container 18S1 was packed with MT57 dioxide. This container has been surveilled 2 years now. The item is in a metal slip lid container with a PVC bag around it. Samples of a possible alternate bag-out bag material were placed in SAVY in FY17 and during this year's surveillance, one of the samples was removed so that the condition of the alternative material could be evaluated.

Table 15. Unpacking Data for Sample 18S1

\begin{tabular}{|c|c|}
\hline Surveillance sample number & $18 S 1$ \\
\hline Operator & Adrian Sanchez \\
\hline Date & 03/1/2018 \\
\hline $\begin{array}{l}\text { Cont. found outside SAVY } \\
\qquad 4000\end{array}$ & No \\
\hline Cont. found inside SAVY 4000 & No \\
\hline Overall package weight & $11117.1 \mathrm{~g}$ \\
\hline Body Serial & 111308080 \\
\hline Lid Serial & 111308080 \\
\hline LOT ID & BLO-39-11-16 \\
\hline Pewter Internal Shield & No \\
\hline Pewter Outer Shield & Yes \\
\hline Outer container Condition & Good \\
\hline O-ring installed & Yes \\
\hline Bag-out bag present & Yes \\
\hline Failed Bag-out bag & No \\
\hline Liquid observed & No \\
\hline Condition of old bag-out bag & Good \\
\hline Type of inner & 3 Qt Hagan \\
\hline Condition of Inner & Ok-Little rust \\
\hline Failed metal inner & No \\
\hline Bag-out bag replace & No \\
\hline Inner replace & No \\
\hline $\begin{array}{l}\text { Beta/Gamma Dose (at } \\
\text { lid/collar of SAVY 4000) }\end{array}$ & $25.0 \mathrm{mrem} / \mathrm{hr}$ \\
\hline Beta/Gamma Dose (at $30 \mathrm{~cm}$ ) & $6.0 \mathrm{mrem} / \mathrm{hr}$ \\
\hline Neutron Dose & $18.0 \mathrm{mrem} / \mathrm{hr}$ \\
\hline
\end{tabular}




\section{Comments}

There is residue around the inside wall of the container (Figure 4-13) and corrosion around the weld inside the container (Figure 4-14). The O-ring passed the visual inspection, but did have small inclusions but not enough to comprise the seal. The bagout bag was reported in good condition.

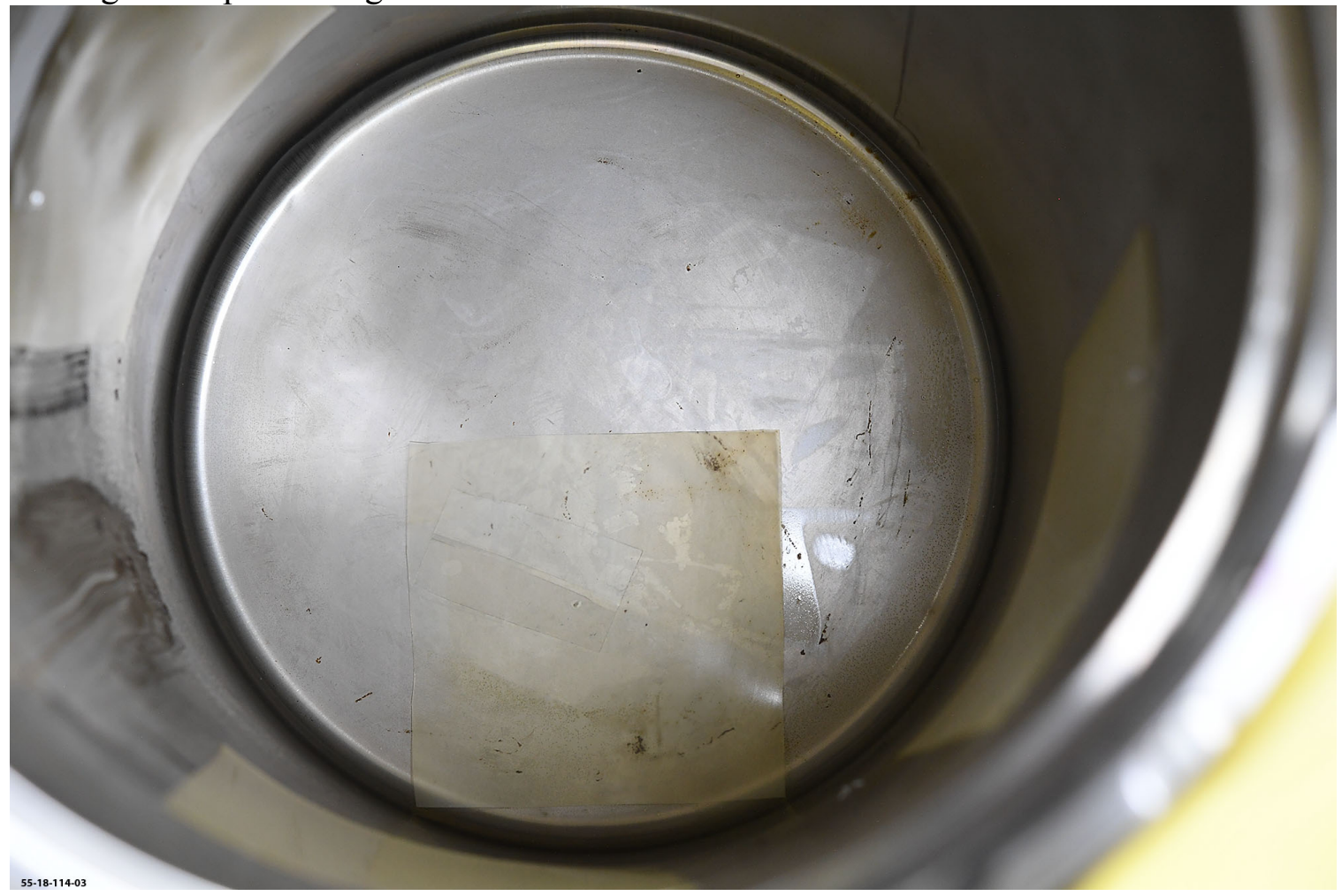

Figure 4-13. Alternate bag-out bag sample in $18 \mathrm{~S} 1$. 


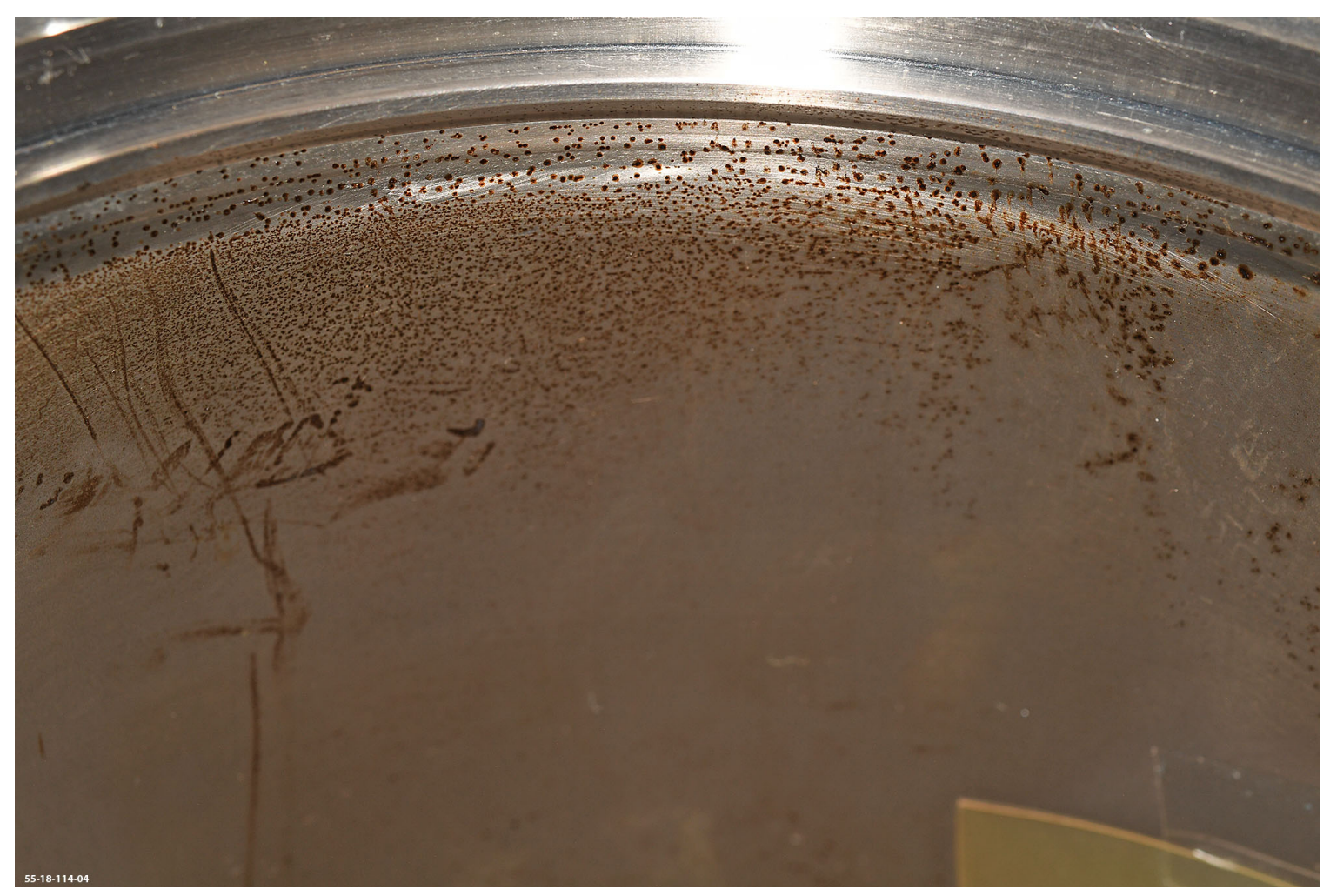

Figure 4-14. Corrosion around weld in $18 \mathrm{~S} 1$.

\subsubsection{SAVY 4000 Container, Surveillance, Sample 18S2, SN 031105052 ,}

CXLOX082911, MT52, 787g, C21, Compound Residue, .1 W, 6.1 years

Container 18S2 was packaged with MT52 compound residue. This container has been surveilled for 4 years now. The inner item has a PVC bag around it.

Table 16. Unpacking Data for Sample $18 \mathrm{S2}$

\begin{tabular}{|c|c|}
\hline Surveillance sample number & $\mathbf{1 8 S 2}$ \\
\hline Operator & Adrian Sanchez \\
\hline Date & $03 / 1 / 2018$ \\
\hline $\begin{array}{c}\text { Cont. found outside SAVY } \\
\mathbf{4 0 0 0}\end{array}$ & No \\
\hline Cont. found inside SAVY 4000 & No \\
\hline Overall package weight & $6763.1 \mathrm{~g}$ \\
\hline Body Serial & 031105052 \\
\hline Lid Serial & 031105052 \\
\hline LOT ID & CXLOX082911 \\
\hline Pewter Internal Shield & No \\
\hline Pewter Outer Shield & Yes \\
\hline Outer container Condition & Good \\
\hline O-ring installed & Yes \\
\hline Bag-out bag present & Yes \\
\hline
\end{tabular}




\begin{tabular}{|c|c|}
\hline Failed Bag-out bag & No \\
\hline Liquid observed & No \\
\hline Condition of old bag-out bag & Good \\
\hline Type of inner & Unable to verify \\
\hline Condition of Inner & Good \\
\hline Failed metal inner & No \\
\hline Bag-out bag replace & No \\
\hline Inner replace & No \\
\hline $\begin{array}{c}\text { Beta/Gamma Dose (at } \\
\text { lid/collar of SAVY 4000) }\end{array}$ & $30.0 \mathrm{mrem} / \mathrm{hr}$ \\
\hline Beta/Gamma Dose (at $\mathbf{3 0} \mathrm{cm}$ ) & $3.5 \mathrm{mrem} / \mathrm{hr}$ \\
\hline Neutron Dose & $5.0 \mathrm{mrem} / \mathrm{hr}$ \\
\hline Comments & \\
\hline
\end{tabular}

Thumb latch is a little sticky but still works. The bag-out bag was reported to be in good condition.

\subsubsection{SAVY 4000 Container, Surveillance, Sample 18S3b, SN 111308059, XAP6 (Outer), MT53, 69g, M53, Unalloyed Metal, $0.21 \mathrm{~W}, 2$ years}

Container $18 \mathrm{~S} 3 \mathrm{~b}$ was packed with MT53 metal. This container is the outer most container and contains a capped SAVY 4000, thus no bag-out bag/gases are in direct contact with 111308059.

Table 17. Unpacking Data for Sample 18S3b

\begin{tabular}{|c|c|}
\hline Surveillance sample number & $\mathbf{1 8 S 3 \mathbf { b }}$ \\
\hline Operator & Adrian Sanchez \\
\hline Date & $03 / 1 / 2018$ \\
\hline $\begin{array}{c}\text { Cont. found outside SAVY } \\
\mathbf{4 0 0 0}\end{array}$ & No \\
\hline Cont. found inside SAVY 4000 & $12325.4 \mathrm{~g}$ \\
\hline Overall package weight & 111308059 \\
\hline Body Serial & 111308059 \\
\hline Lid Serial & XAP6 \\
\hline LOT ID & Yes \\
\hline Pewter Internal Shield & Yes \\
\hline Pewter Outer Shield & Good \\
\hline Outer container Condition & Yes \\
\hline O-ring installed & Yes \\
\hline Bag-out bag present & No \\
\hline Failed Bag-out bag & No \\
\hline Liquid observed & Good \\
\hline Condition of old bag-out bag & Unable to verify \\
\hline Type of inner & Ok \\
\hline Condition of Inner
\end{tabular}




\begin{tabular}{|c|c|}
\hline Failed metal inner & No \\
\hline Bag-out bag replace & No \\
\hline Inner replace & No \\
\hline $\begin{array}{c}\text { Beta/Gamma Dose (at } \\
\text { lid/collar of SAVY 4000) }\end{array}$ & $70.0 \mathrm{mrem} / \mathrm{hr}$ \\
\hline Beta/Gamma Dose (at $\mathbf{3 0} \mathrm{cm}$ ) & $10.0 \mathrm{mrem} / \mathrm{hr}$ \\
\hline Neutron Dose & $35.0 \mathrm{mrem} / \mathrm{hr}$ \\
\hline Comments & \\
\hline
\end{tabular}

Discoloration on the inner side of the locking ring.

\subsubsection{SAVY 4000 Container, Surveillance, Sample 18S4, SN 031105051, ROTRBJ-1C1, MT52, 452g, R26, $1.2 \mathrm{~W}, 6.6$ years}

Container 18S4 was packaged with MT52 dioxide. This container has been surveilled for 4 years now. The item is in a metal slip lid container with a PVC bag around it.

Table 18. Unpacking Data for Sample 18S4

\begin{tabular}{|c|c|}
\hline Surveillance sample number & $\mathbf{1 8 S 4}$ \\
\hline Operator & Adrian Sanchez \\
\hline Date & $03 / 14 / 2017$ \\
\hline $\begin{array}{c}\text { Cont. found outside SAVY } \\
\text { 4000 }\end{array}$ & No \\
\hline Cont. found inside SAVY 4000 & No \\
\hline Overall package weight & $9208.6 \mathrm{~g}$ \\
\hline Body Serial & 031105051 \\
\hline Lid Serial & 031105051 \\
\hline LOT ID & ROTRBJ-1C1 \\
\hline Pewter Internal Shield & No \\
\hline Pewter Outer Shield & Yes \\
\hline Outer container Condition & Good \\
\hline O-ring installed & Yes \\
\hline Bag-out bag present & Yes \\
\hline Failed Bag-out bag & No \\
\hline Liquid observed & No \\
\hline Condition of old bag-out bag & Good \\
\hline Type of inner & Unable to verify \\
\hline Condition of Inner & Good \\
\hline Failed metal inner & No \\
\hline Bag-out bag replace & No \\
\hline Inner replace & No \\
\hline Beta/Gammallar of SAVY 4000) & $11.0 \mathrm{mrem} / \mathrm{hr}$ \\
\hline Neutron Dose & $2.0 \mathrm{mrem} / \mathrm{hr}$ \\
\hline (ad & $8.0 \mathrm{mrem} / \mathrm{hr}$ \\
\hline
\end{tabular}




\section{Comments}

Sticky thumb latch and small amounts of residue throughout the inside of the container. (Figure 4-15 \& Figure 4-16). Small dimple on bottom of the container. The o-ring had a small pit, but it was not big enough to compromise the seal. The bag-out bag was reported in good condition.

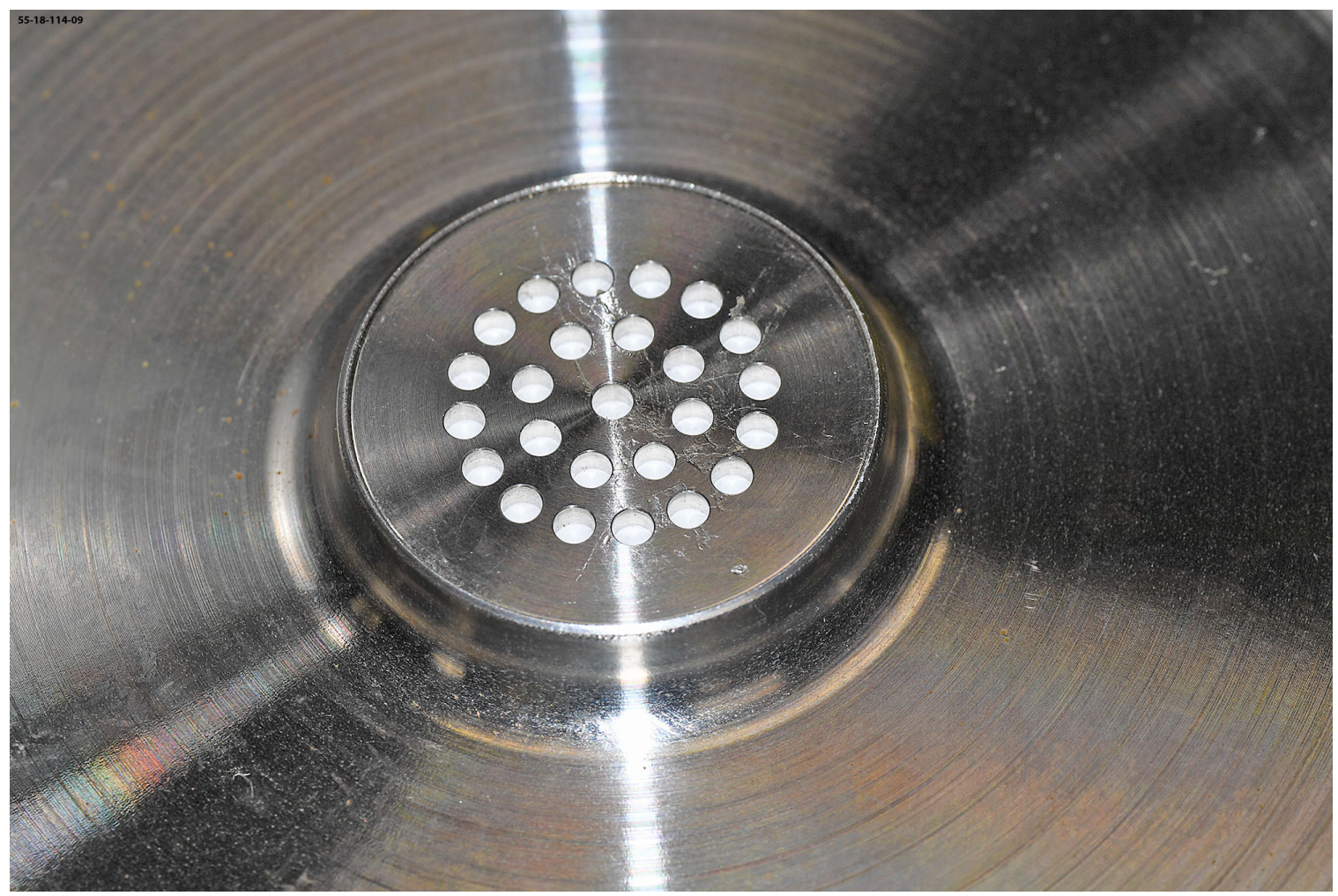

Figure 4-15. Underside of lid on 1854. 


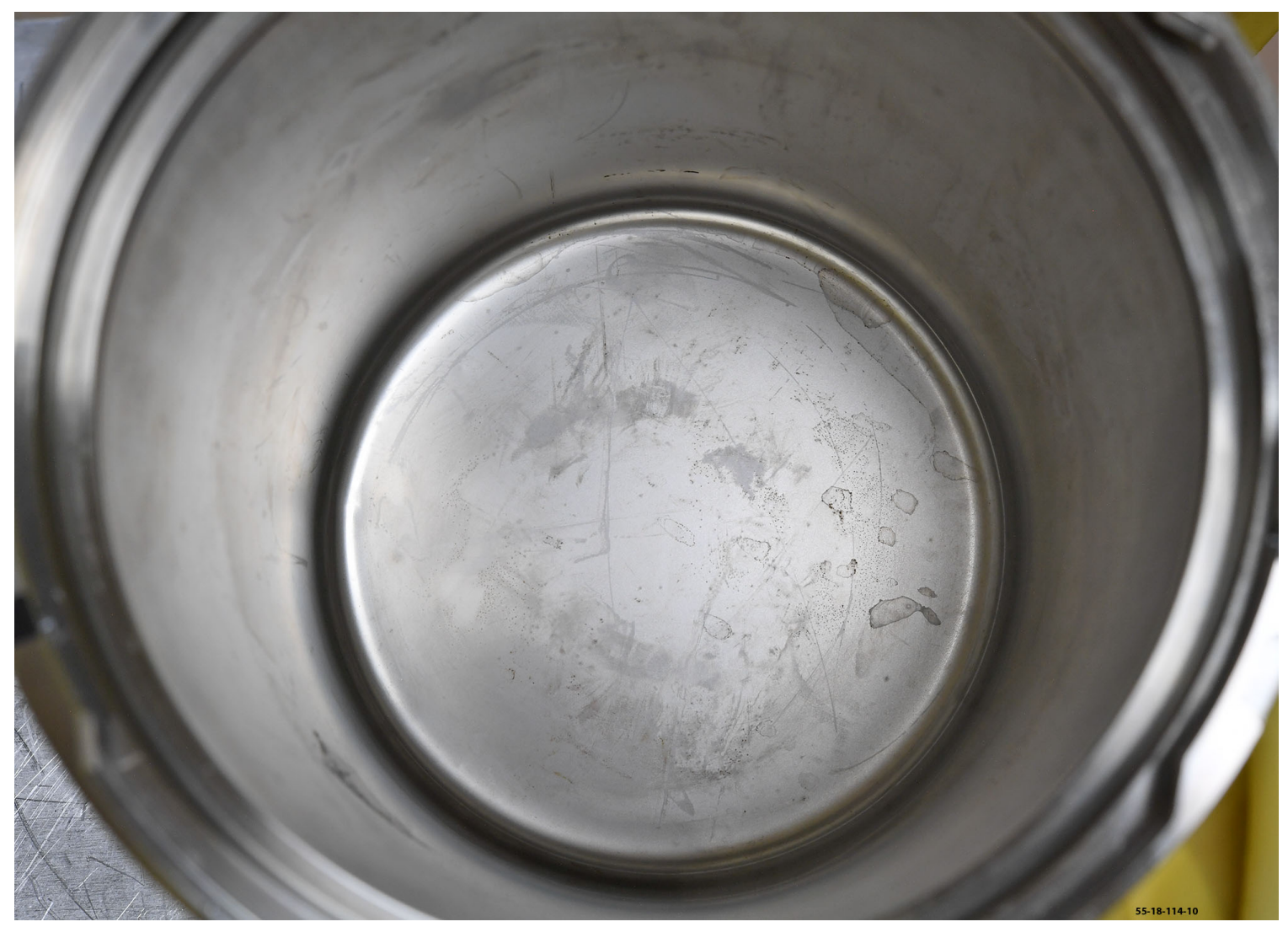

Figure 4-16. Residue on the bottom inside of $18 \mathrm{~S} 4$

\subsubsection{SAVY 4000 Container, Surveillance, Sample 18S5, SN 121103083, XBLSCL1217, MT52 + MT44, 178g, R83, MSE Salt, 2.9 W, 4.9 years}

Container 18S5 was packed with MT52 and MT44 MSE salt. This container has been surveilled for 4 years now. The item is in a metal slip lid container with a PVC bag around it.

Table 19. Unpacking Data for Sample $18 S 5$

\begin{tabular}{|c|c|}
\hline Surveillance sample number & $\mathbf{1 8 S 5}$ \\
\hline Operator & Adrian Sanchez \\
\hline Date & $3 / 1 / 2018$ \\
\hline $\begin{array}{c}\text { Cont. found outside SAVY } \\
\mathbf{4 0 0 0}\end{array}$ & No \\
\hline Cont. found inside SAVY 4000 & No \\
\hline Overall package weight & 4275.4 g \\
\hline Body Serial & 121103083 \\
\hline Lid Serial & 121103083 \\
\hline LOT ID & XBLSCL1217 \\
\hline Pewter Internal Shield & No \\
\hline Pewter Outer Shield & Yes \\
\hline Outer container Condition & Good \\
\hline
\end{tabular}




\begin{tabular}{|c|c|}
\hline O-ring installed & Yes \\
\hline Bag-out bag present & Yes \\
\hline Failed Bag-out bag & No \\
\hline Liquid observed & No \\
\hline Condition of old bag-out bag & Good \\
\hline Type of inner & Metal slip lid \\
\hline Condition of Inner & Ok \\
\hline Failed metal inner & No \\
\hline Bag-out bag replace & No \\
\hline Inner replace & No \\
\hline $\begin{array}{c}\text { Beta/Gamma Dose (at } \\
\text { lid/collar of SAVY 4000) }\end{array}$ & $20.0 \mathrm{mrem} / \mathrm{hr}$ \\
\hline Beta/Gamma Dose (at $\mathbf{3 0} \mathrm{cm})$ & $3 \mathrm{mrem} / \mathrm{hr}$ \\
\hline Neutron Dose & $5 \mathrm{mrem} / \mathrm{hr}$ \\
\hline Comments & \\
\hline
\end{tabular}

There were no external issues found during the inspection. Early signs of corrosion was found throughout the inside of the container (Figure 4-17). The anodizing color on the locking ring has changed which becomes apparent by examining other areas of the locking ring. There is a sign/scratch on the locking ring of being dinged or hit against another object. The bag was reported in good condition. 


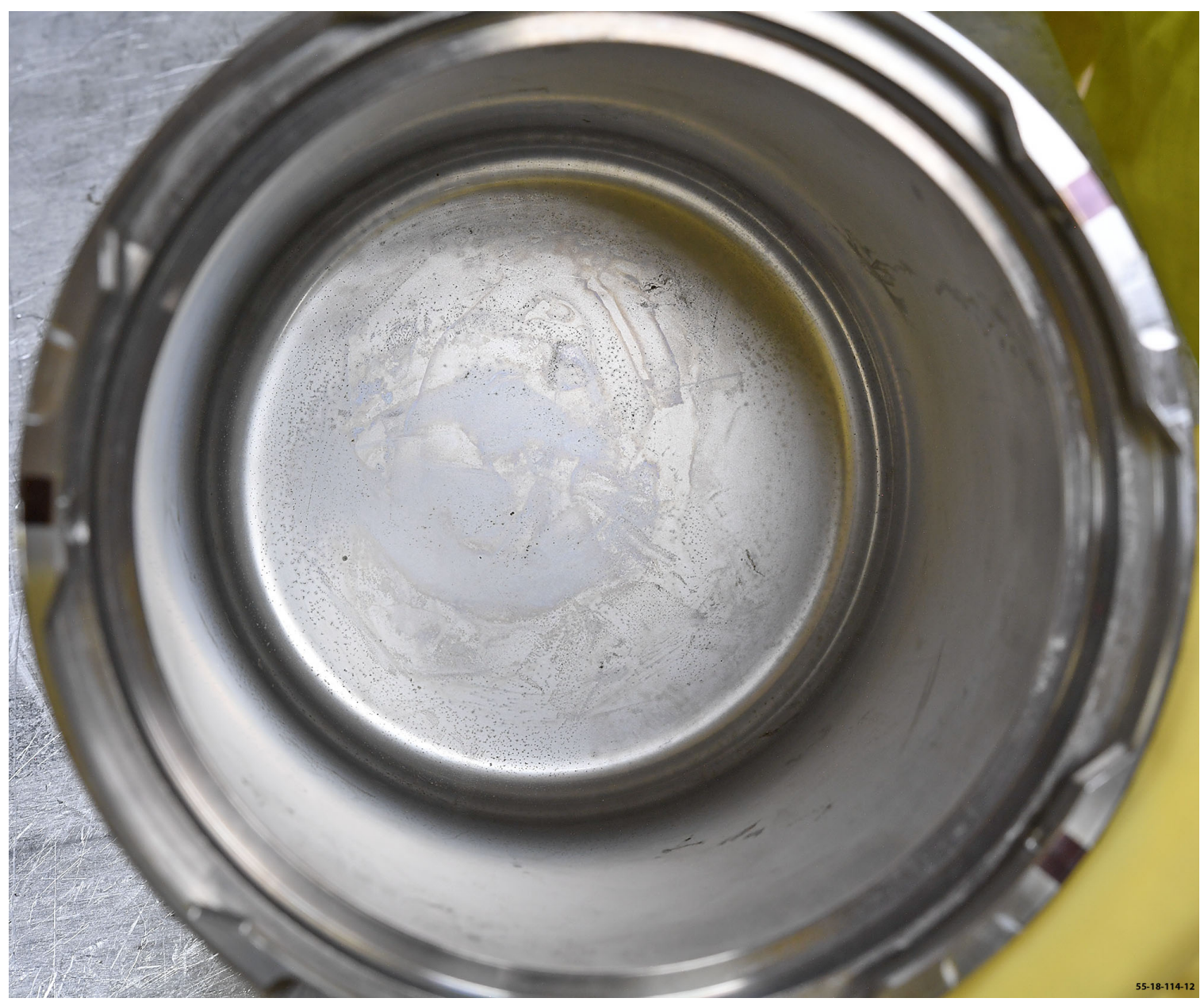

Figure 4-17. Small spots of corrosion seen on $18 \mathrm{~S} 5$.

4.2.1.6 SAVY 4000 Container, Surveillance, Sample 18S6, SN 121103062, SLTF3123A, MT52, 1900g, R42, DOR Salt, $2.9 \mathrm{~W}, 5.4$ years

Container 18S6 was packed with MT52 DOR salt. This container was meant to be sent through all of the surveillance tests, but during the unpacking procedure contamination was found on the inside of the SAVY. The container was introduced into the glovebox line. This would have been the first time 121103062 would have been surveilled.

Table 20. Unpacking Data for Sample $18 \mathrm{S6}$

\begin{tabular}{|c|c|}
\hline Surveillance sample number & $\mathbf{1 8 S 6}$ \\
\hline Operator & 8/16/2018 \\
\hline Date & No \\
\hline $\begin{array}{c}\text { Cont. found outside SAVY } \\
\text { Co00 found inside SAVY 4000 }\end{array}$ & Yes \\
\hline Overall package weight & $4275.4 \mathrm{~g}$ \\
\hline
\end{tabular}




\begin{tabular}{|c|c|}
\hline Body Serial & 121103062 \\
\hline Lid Serial & 121103062 \\
\hline Pewter Internal Shield & SLTF3123A \\
\hline Pewter Outer Shield & Yes \\
\hline Outer container Condition & Good \\
\hline O-ring installed & Yes \\
\hline Bag-out bag present & Yes \\
\hline Failed Bag-out bag & Yes \\
\hline Liquid observed & No \\
\hline Condition of old bag-out bag & Cracked \\
\hline Type of inner & Metal slip lid \\
\hline Condition of Inner & Ok \\
\hline Failed metal inner & No \\
\hline Bag-out bag replace & No \\
\hline Inner replace & No \\
\hline Beta/Gamma Dose (at & N/A \\
\hline lid/collar of SAVY 4000) & N/A \\
\hline Beta/Gamma Dose (at 30 cm) & N/A \\
\hline Neutron Dose & \\
\hline Comments & \\
\hline
\end{tabular}

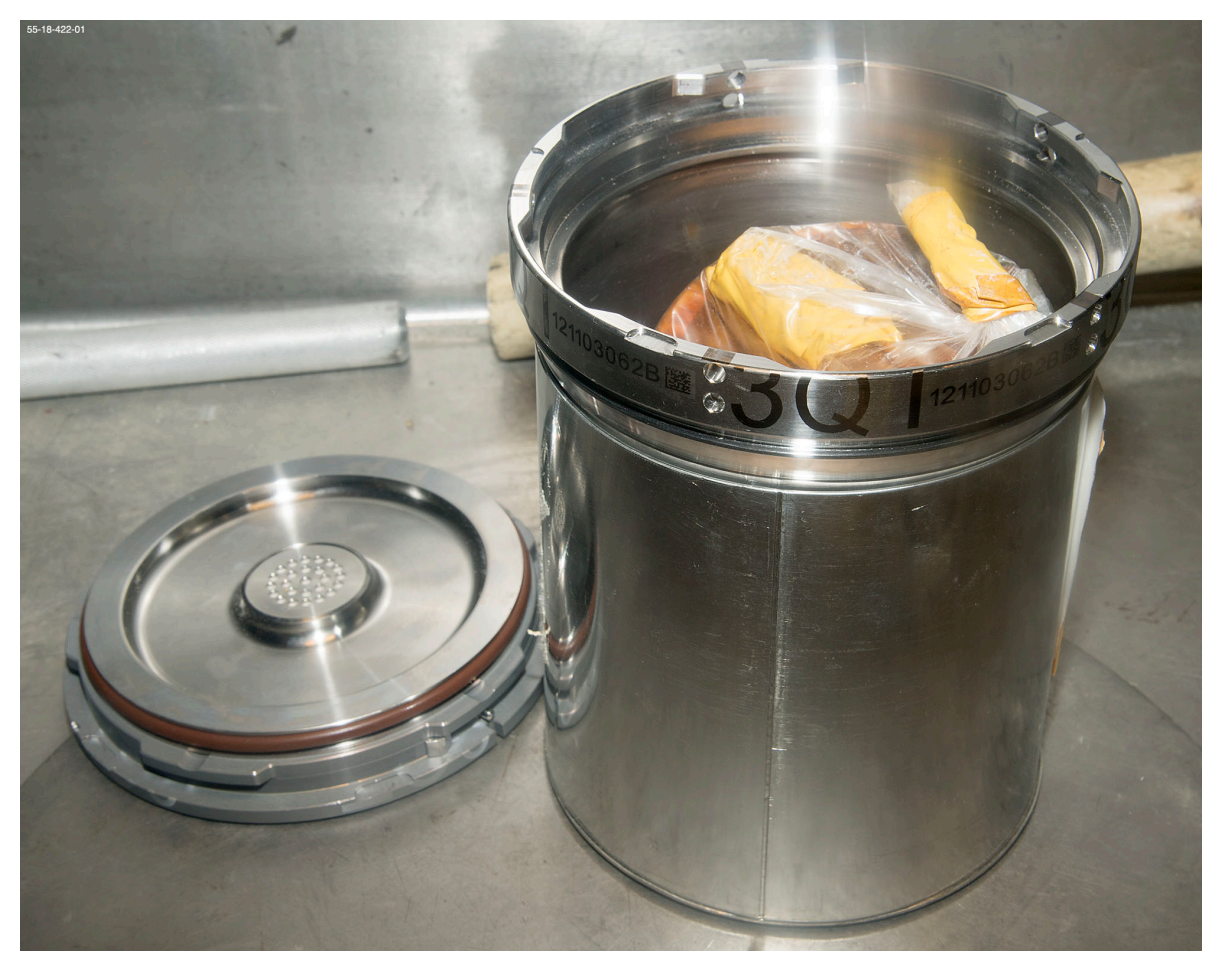

Figure 4-18. Packaging configuration of $18 \mathrm{S6}$. 


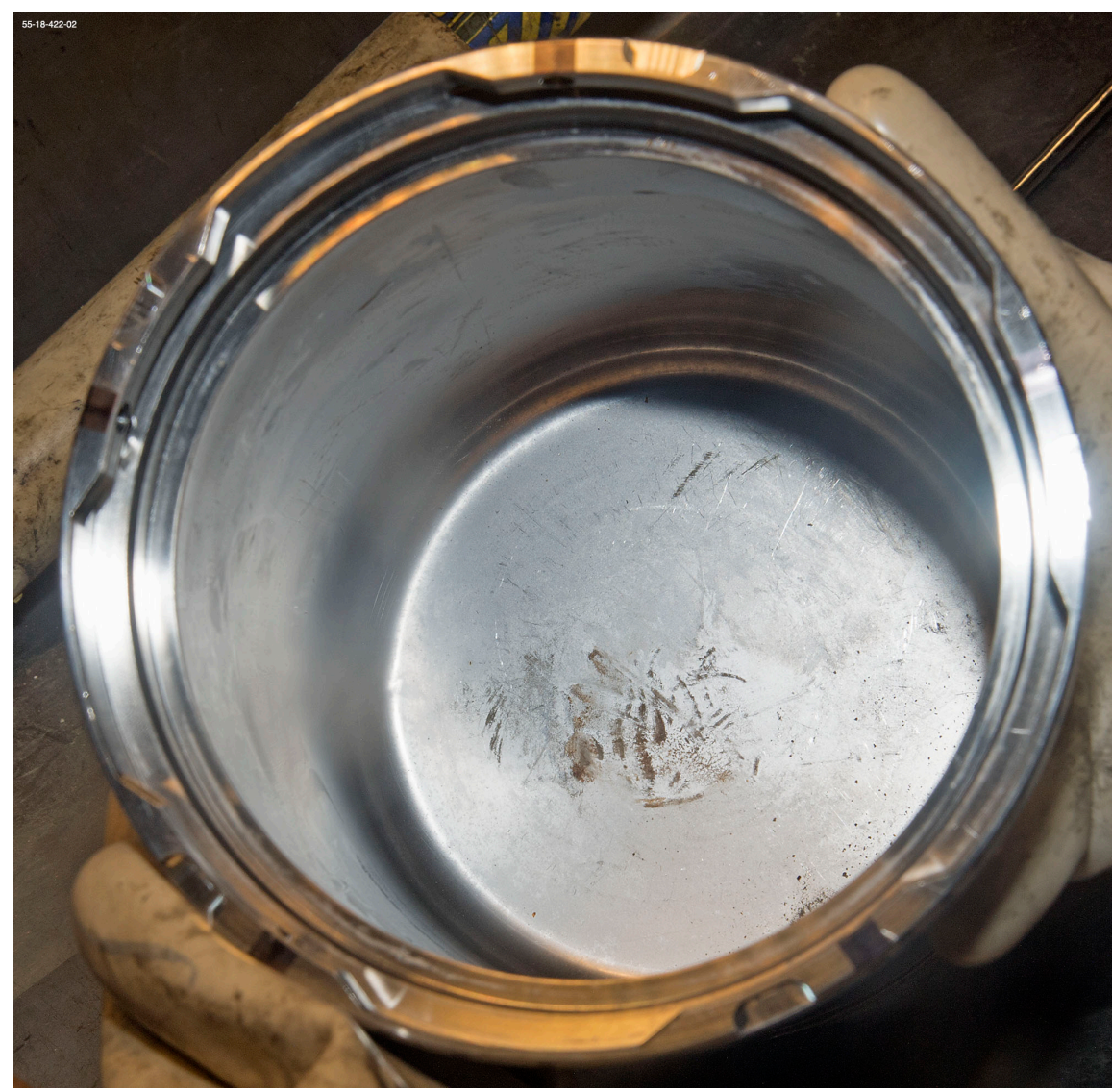

Figure 4-19. Small amount of corrosion at the bottom inside of $18 \mathrm{S6}$.

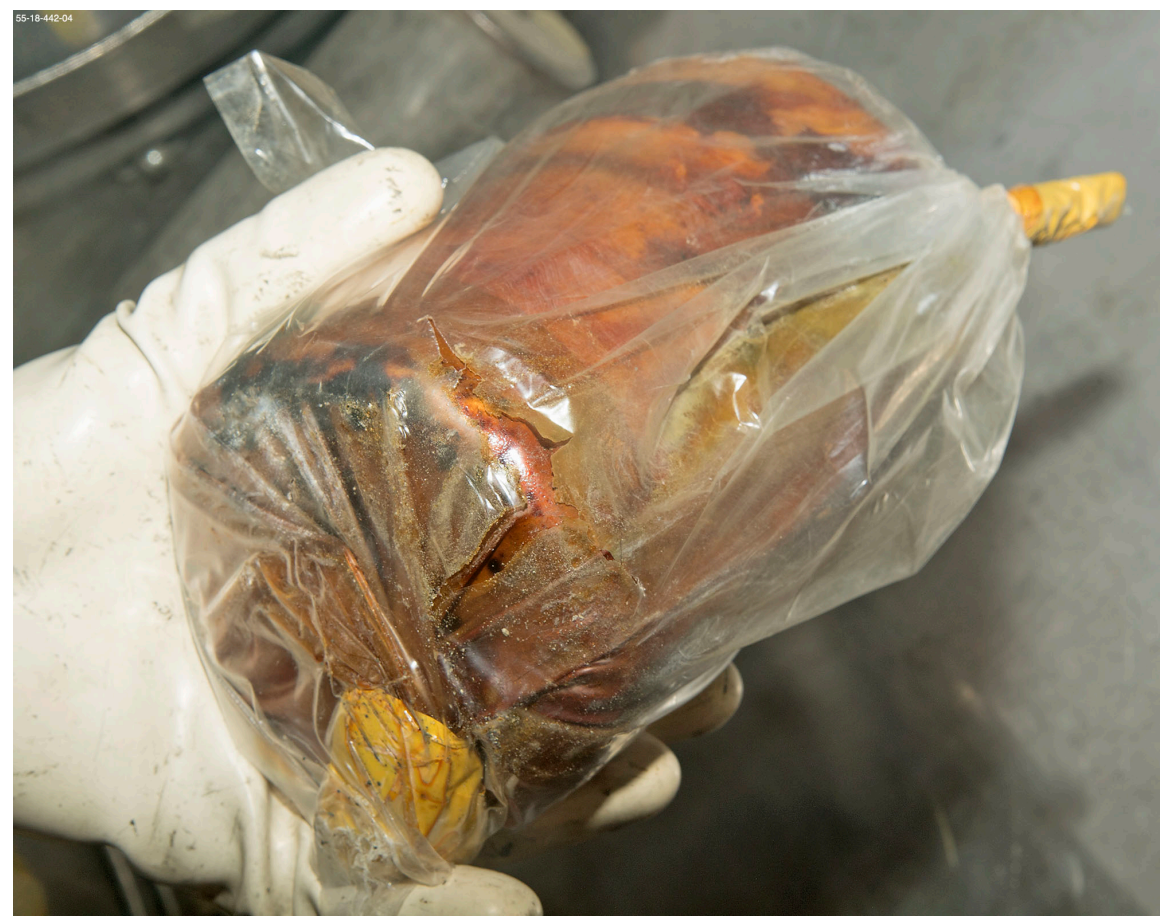

Figure 4-20. Small tear on the bottom of the bag-out bag that was in $18 \mathrm{~S} 6$. 


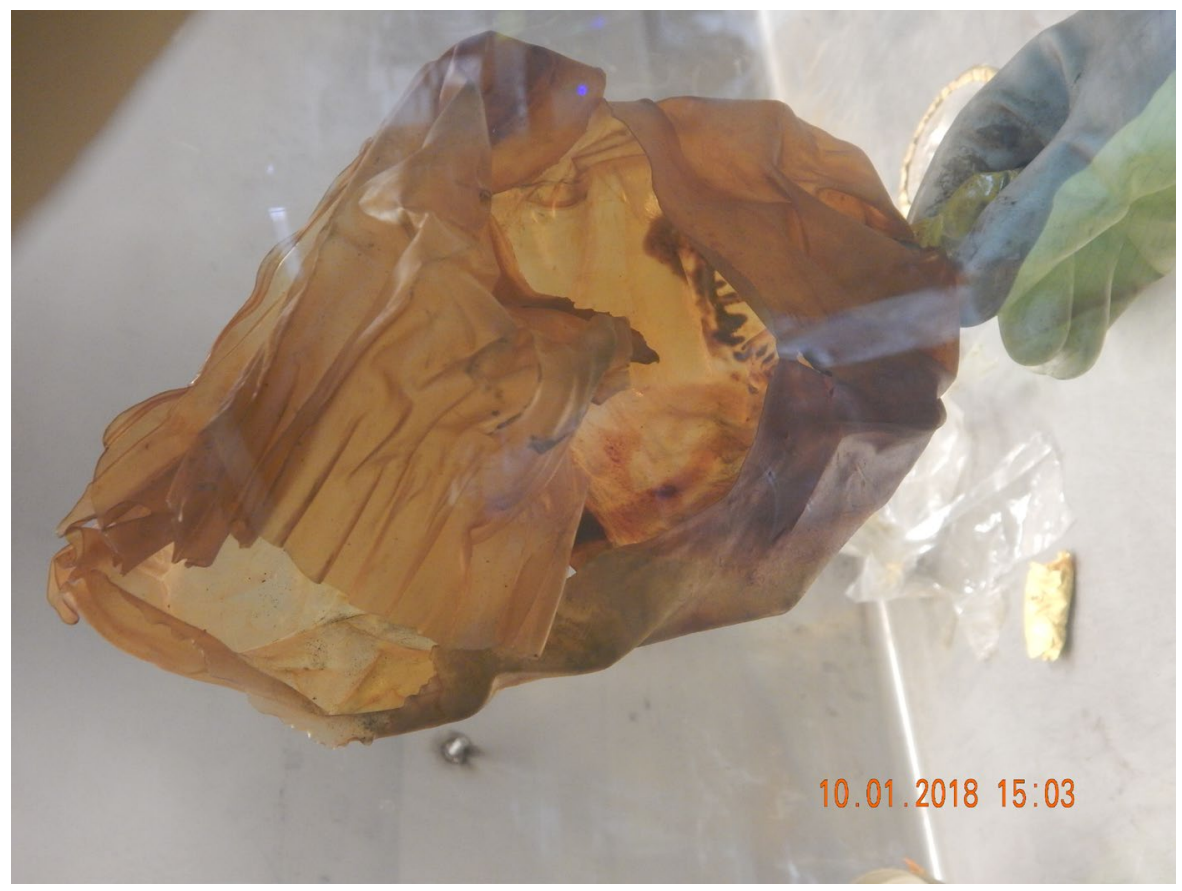

Figure 4-21. Condition of inner PVC bag on $18 \mathrm{~S} 6$.

Contamination was found on the inside of the SAVY container. A tear was observed in the outer bag (probably polyethylene), and a crack was observed at the bottom of the inner bag (probably PVC), which is likely the source of the contamination. It was also observed that the lid of the slip lid container was not seated on the top of the container. This may have been due to an inappropriate choice of inner container for the size of the item at the time of packaging. It might also have been caused by material expansion, for example, oxidative expansion of plutonium metal. Once the inner contents were removed from the SAVY small amounts of corrosion was seen on the inside bottom of the container.

\subsubsection{SAVY 4000 Container, Surveillance, Sample 18S7, SN 091205130, SAMPCAN2, MT83, 34.8g, C21, Dioxide, $16.6 \mathrm{~W}, 3.8$ years}

Container 18S7 was packaged with MT83 dioxide. SAVY 091205130 had never been evaluated before. This container was introduced by the operators due to the risk that the inside of the SAVY could have been contaminated. The items were in a metal slip lid container with a PVC bag around it.

Table 21. Unpacking Data for Sample $18 \mathrm{~S} 7$

\begin{tabular}{|c|c|}
\hline Surveillance sample number & $\mathbf{1 8 S 7}$ \\
\hline Operator & \\
\hline Date & $6 / 5 / 2018$ \\
\hline $\begin{array}{c}\text { Cont. found outside SAVY } \\
\mathbf{4 0 0 0}\end{array}$ & No \\
\hline
\end{tabular}




\begin{tabular}{|c|c|}
\hline Cont. found inside SAVY 4000 & Unknown \\
\hline Overall package weight & Unknown \\
\hline Body Serial & 091205130 \\
\hline Lid Serial & 091205130 \\
\hline LOT ID & SAMPCAN2 \\
\hline Pewter Internal Shield & No \\
\hline Pewter Outer Shield & No \\
\hline Outer container Condition & Good \\
\hline O-ring installed & Yes \\
\hline Bag-out bag present & Yes \\
\hline Failed Bag-out bag & Unknown \\
\hline Liquid observed & Yes \\
\hline Condition of old bag-out bag & Darkened \\
\hline Type of inner & Metal slip lid \\
\hline Condition of Inner & Good \\
\hline Failed metal inner & No \\
\hline Bag-out bag replace & N/A \\
\hline Inner replace & N/A \\
\hline Beta/Gamma Dose (at \\
lid/collar of SAVY 4000)
\end{tabular}




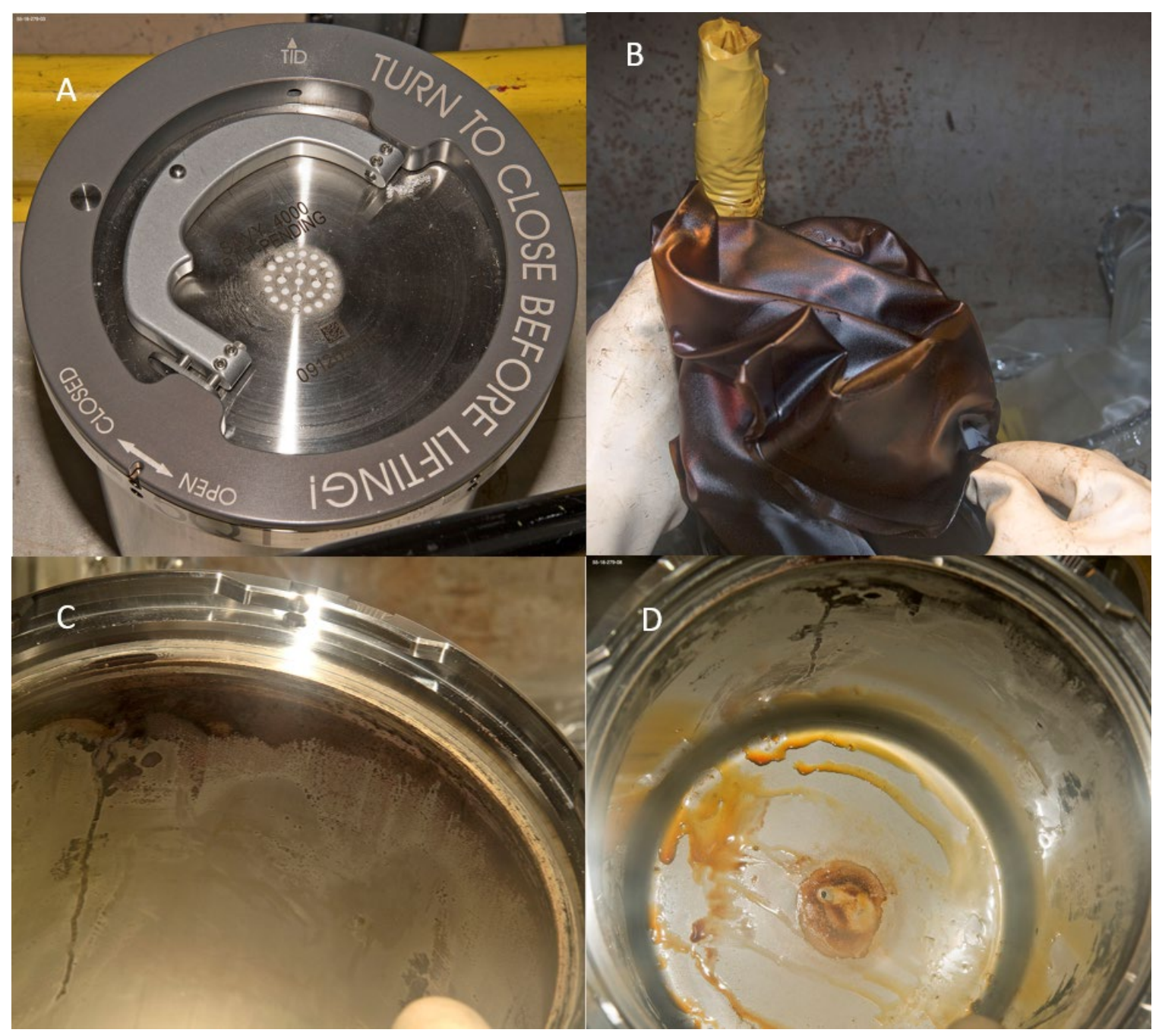

Figure 4-22. Photographs of $18 \mathrm{~S} 7$ at unpacking. (A) External surfaces of container $18 \mathrm{~S} 7$ showing white powder near filter vent. (B) Darkened bag showing signs of heavy degredation. (C) Corrosions does not go past the O-ring sealing surfaces indication that the seal remained intact. (D) Bag-out bag residue covering the bottom surface of the body.

\subsubsection{O-ring Tests}

\subsubsection{Visual Inspection of the O-rings}

Inspections revealed small amounts of dust and debris on a majority of the O-rings. It is unclear whether this dirt was introduced during the use of the container or during manipulations involved in surveillance. In either case, the dirt was easily removed with an alcohol wipe. No flaws were found either the surveillance containers or the transfer containers.

\subsubsection{Container Leak Rate Tests}

The leak rate results for storage and transfer containers with O-rings installed are shown in Figure 4-22 and Figure 4-23. For storage containers, the measured leak rate ranged between $1.4 \times 10^{-8}$ and $3.0 \times 10^{-8} \mathrm{~atm} \mathrm{cc} / \mathrm{s}$ of helium at 75 Torr into vacuum. Tor transfer 
containers, the leak rate ranged between $2.0 \times 10^{-9}$ and $7.5 \times 10^{-7} \mathrm{~atm} \mathrm{cc} / \mathrm{s}$. Every container passed the leak test, with all measurements being below the failure criterion of $1 \times 10^{-5} \mathrm{~atm} \mathrm{cc} / \mathrm{s}$.
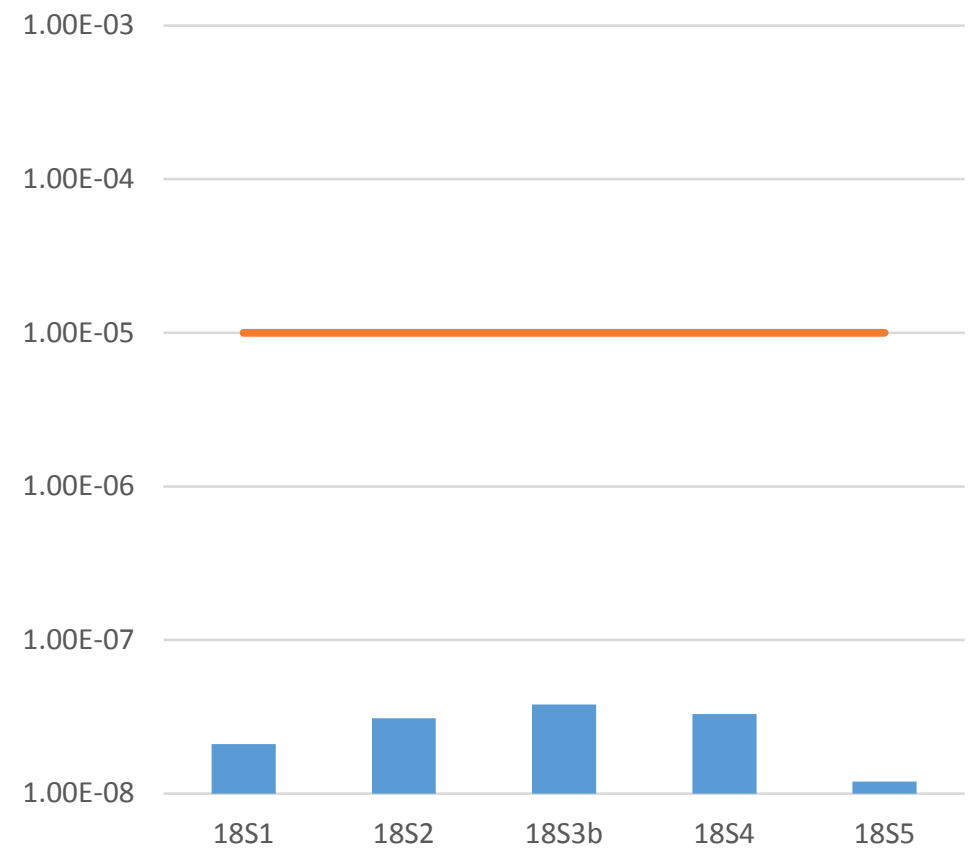

Figure 4-23. Leak rates for surveillance containers, measured for each container are shown as blue bars with the failure criteria shown with the orange line. 
$1.00 \mathrm{E}-03$

1.00E-04

1.00E-05

1.00E-06

Sum of Helium Leak Rate Sum of He Fail

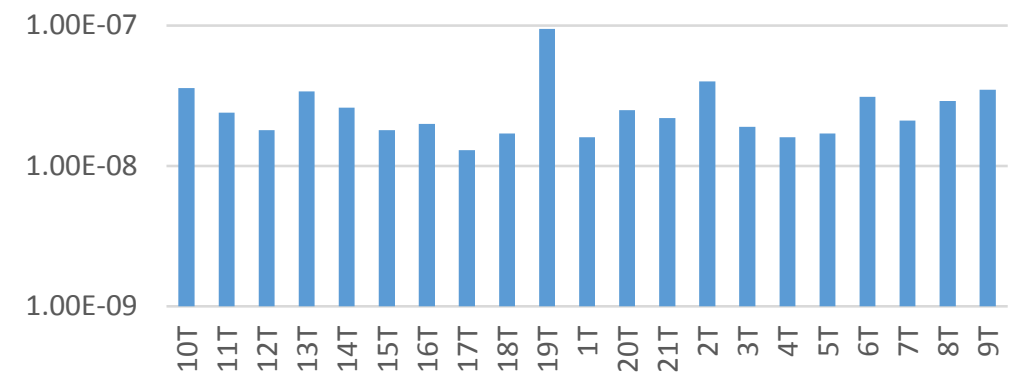

Figure 4-24. Leak rates for transfer containers, measured for each container are shown as blue bars with the failure criteria shown with the orange line.

Leak testing is done as a threshold measurement on a logarithmic scale. Only a single point calibration is done to prepare the instrument for use, though it is checked against another, known leak standard. The calibration value is chosen that is low enough to ensure that the threshold leak - the leak at which failure of a part is determined - is certain to register on the leak detector but high enough to be distinguishable from background. The value of the apparent background leak rate will always decrease during a leak test, as gas is continuously evacuated from the bell jar, so it is not unusual to see leak test measurements lower than the background. This means that the leak test measurement is indistinguishable from the background. These values show measured leak rates that are indistinguishable from the apparent background, and therefore we have confidence containment has been maintained well below the design release rate. The leak test measurement does not have a significant correlation with age, estimated dose, or item thermal power.

\subsubsection{O-ring Hardness Tests}

The 5 surveillance O-rings were found to have hardness of between 57.3 and 57.8, with an average of 57.5 durometer units. The 21 transfer container O-rings had a hardness between 56.2 and 58.6, with an average of 57.2 durometer units. The 28 O-rings used as a baseline in the lifetime extension studies have a hardness of $57.5 \pm 2.62$ durometer units, so the storage and transfer O-rings are similar in hardness to unused O-rings. The 2.62 unit error is the reproducibility standard deviation as given in ASTM D2240. The 
measurements are graphed in Figure 4-24 and Figure 4-25. None of the O-rings are especially hard or soft compared with the baseline, and the measurement do not have a significant correlation with age, estimated dose, or item thermal power. One of the transfer O-ring measurements had a corrupt file and the data could not be found.

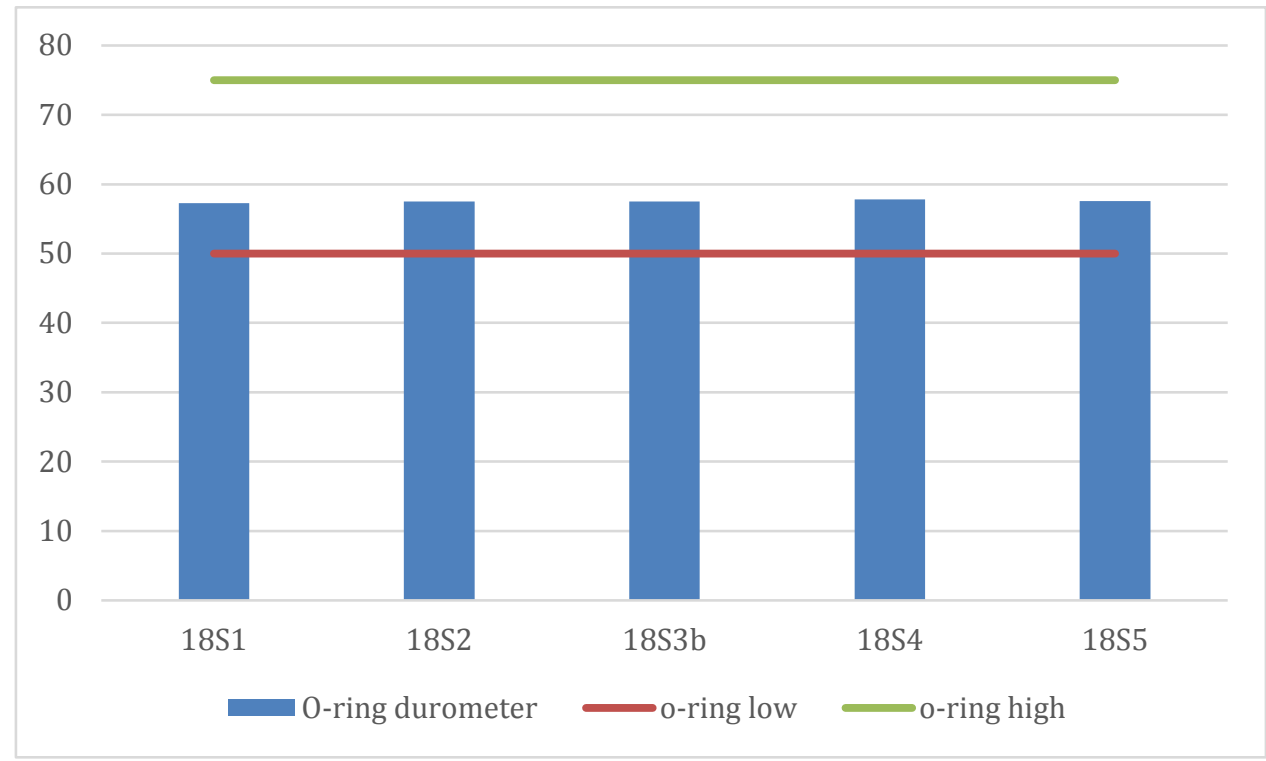

Figure 4-25. Durometer measurements of the surveillance SAVY 4000 Oring with the red and green and red bands showing the upper and lower failure limits. 


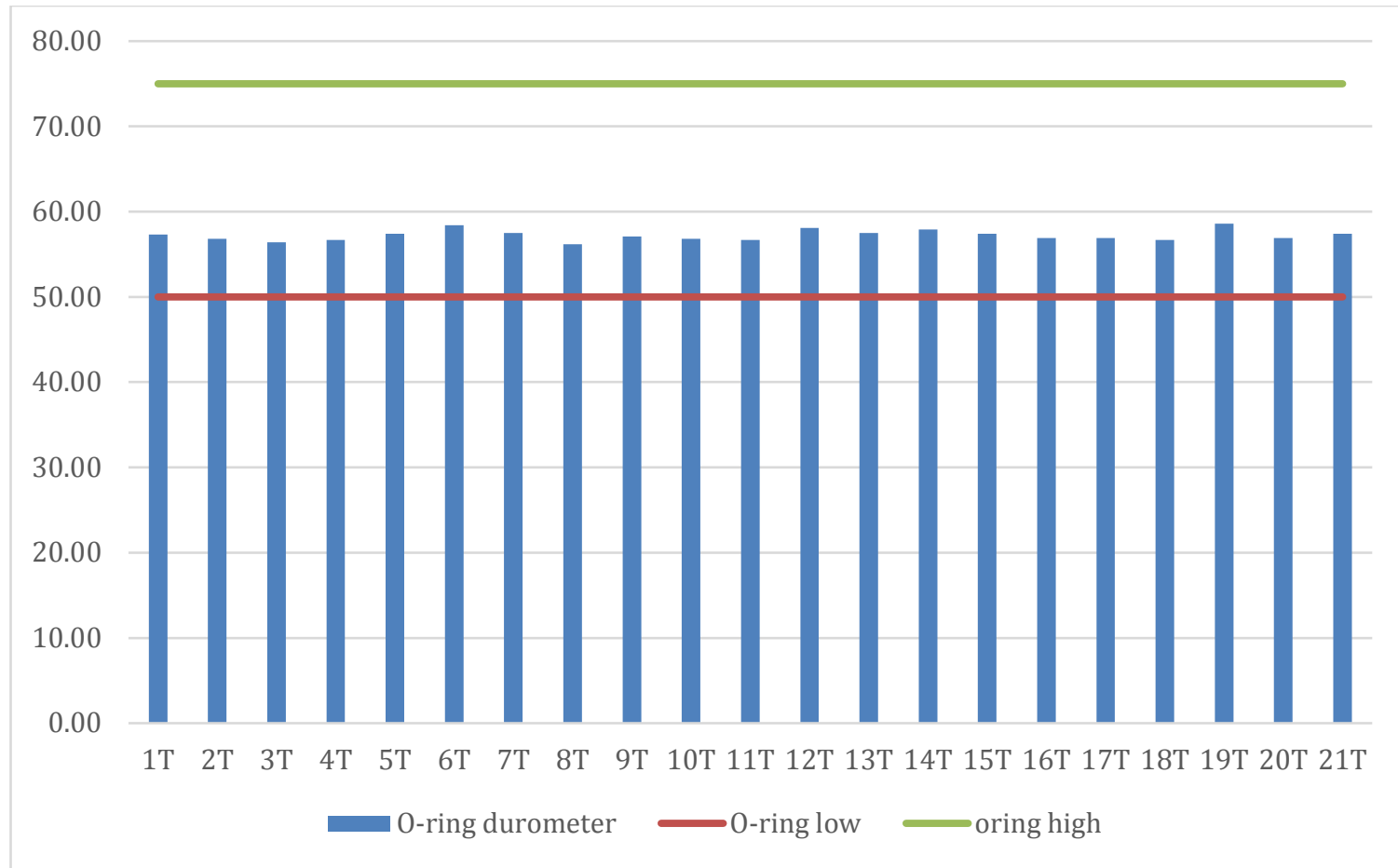

Figure 4-26. Durometer measurements of the transfer container SAVY 4000 $O$-ring with the green and red bands showing the upper and lower failure limits.

\subsubsection{Filter Tests}

\subsubsection{Particle Penetration}

The aerosol data are reported as a percent penetration, also known as the percent leakage. The set of particle penetration measurements is narrowly distributed and very far from the failure criterion as graphed in Figure 4-26 for storage containers and Figure 4-27 for transfer containers. A set of baseline particle penetration measurements does exist, but it is not clear how those measurements, taken on a different type of instrument with a different configuration, relate to the measurements taken in PF-4 with our instrument. Although we may not be able to tell how the particle penetration is changing from baseline, we will be able to track how the particle penetration changes relative to containers' peers in the sample population. 


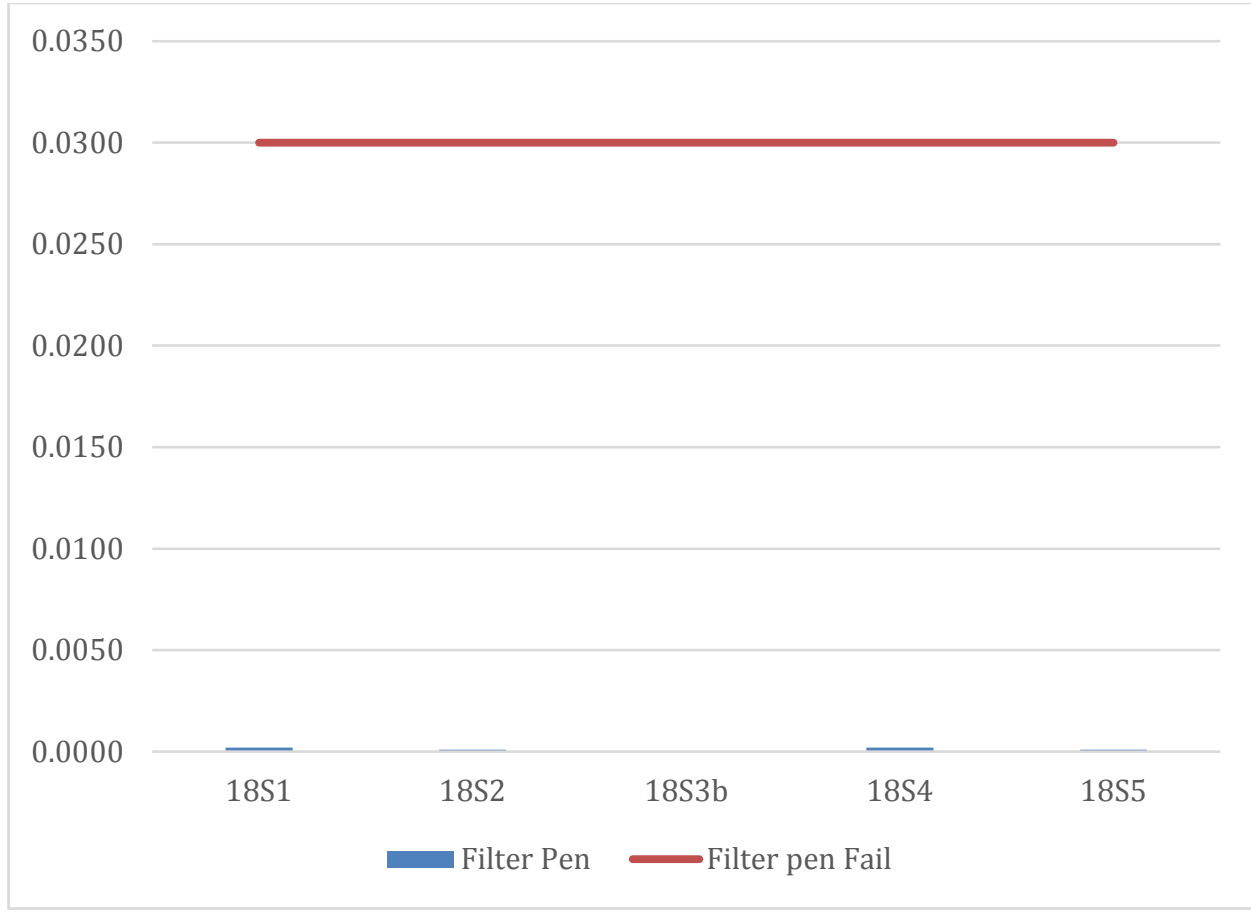

Figure 4-27. Filter particle penetration measurements for storage containers.

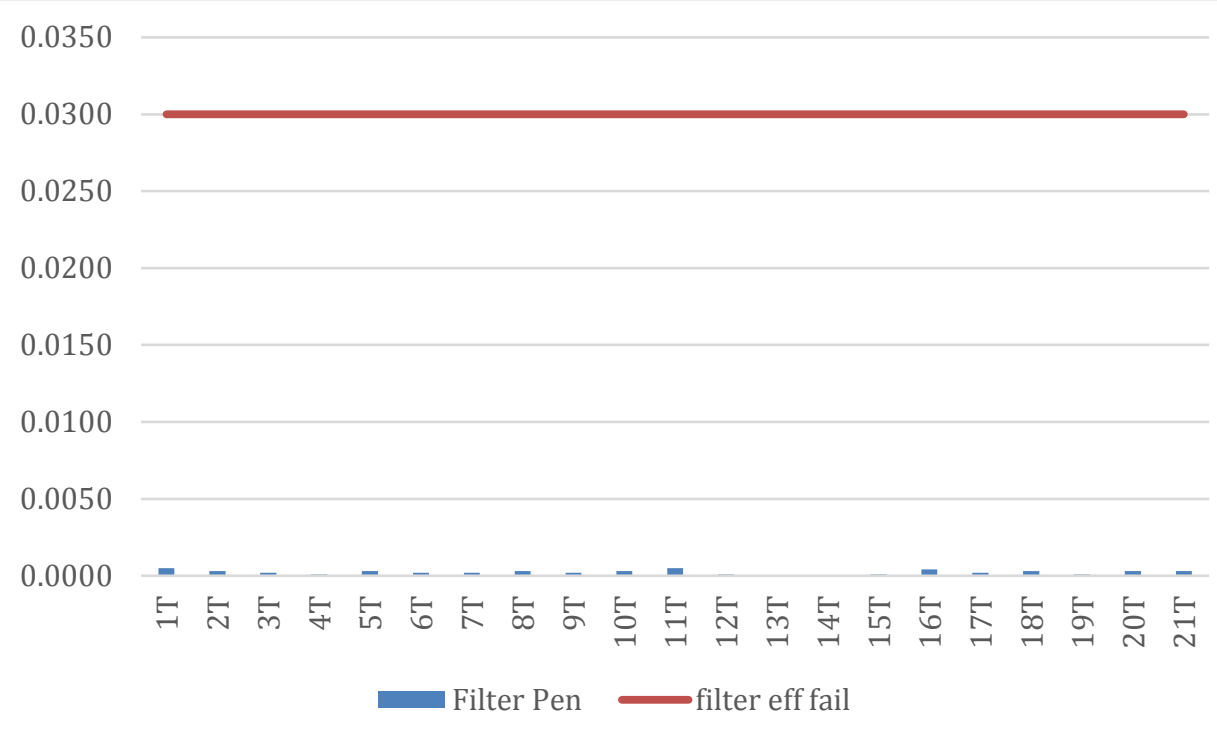

Figure 4-28. Filter particle penetration measurements for transfer containers. 


\subsubsection{Pressure Drop}

The pressure drop measurements have an average of 0.61 in. W.C. \pm 0.09 for storage and 0.66 in. W.C. \pm 0.09 for transfer containers. The results are graphed below in Figure 4-28 and a graph of the measurements is shown in Figure 4-29.

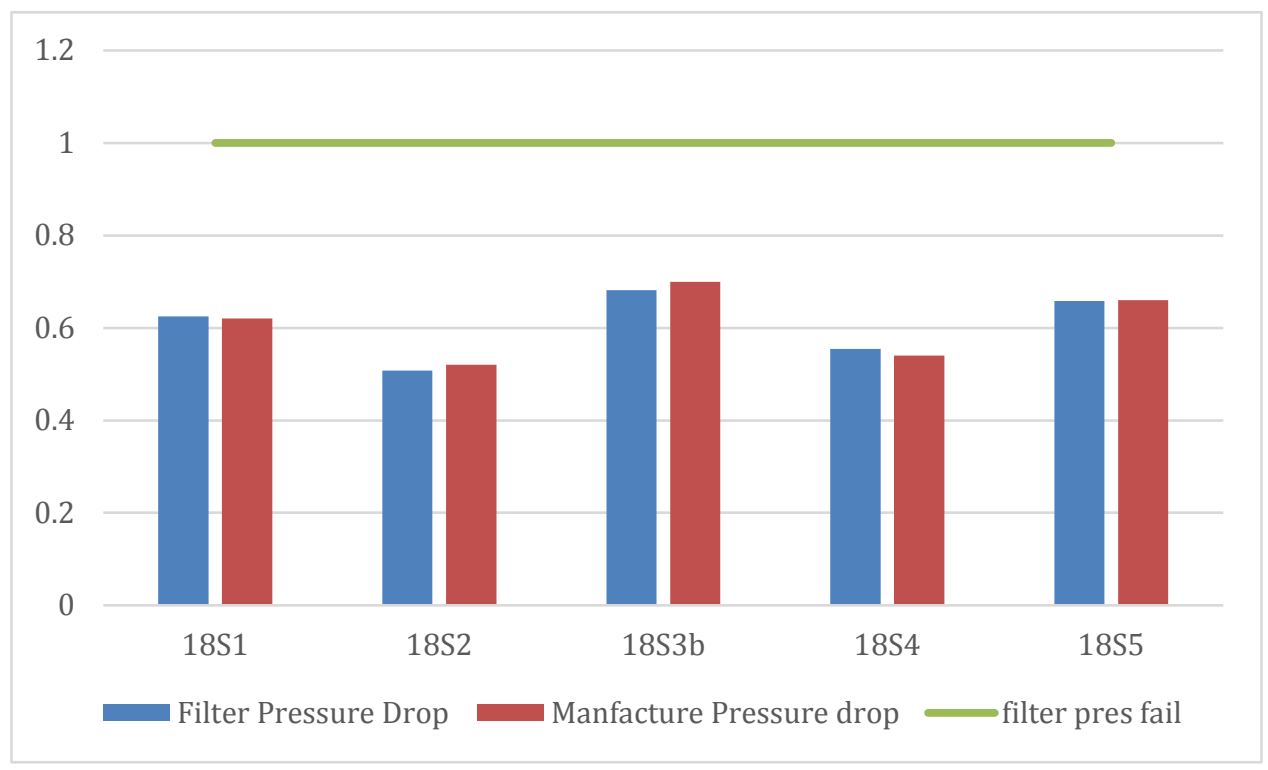

Figure 4-29. Filter pressure drop measurements for storage containers.

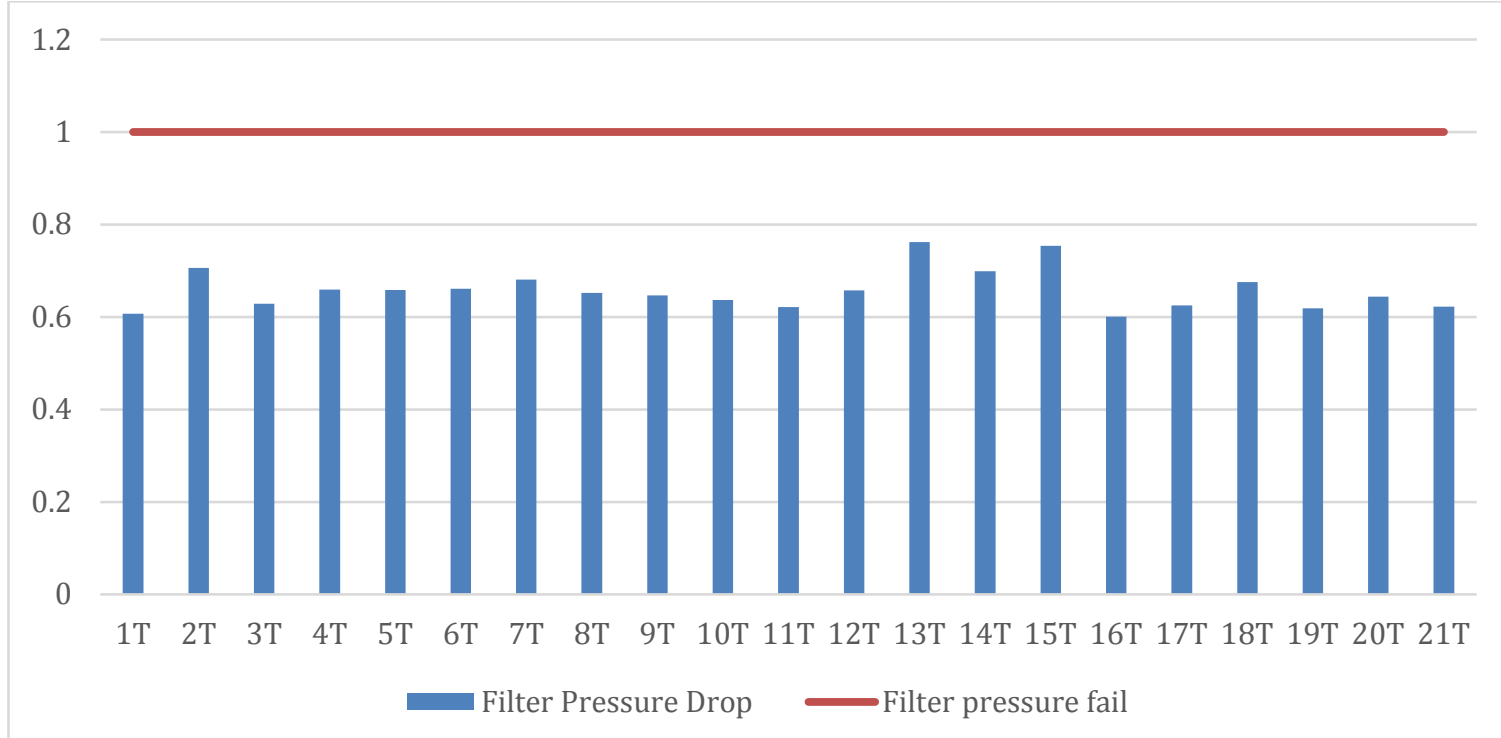

Figure 4-30. Filter pressure drop measurements for transfer containers. 


\subsubsection{Annual Surveillance (NDE only)}

Four of the containers have been designated for annual surveillance in the Surveillance Plan[1]. The relevant data for annual measurements on the four nondestructive evaluation (NDE)-only containers are reproduced in Table 22 thru Table 25. 
Table 22. Relevant data for annual measurements on the four NDE-only containers.

\begin{tabular}{|l|c|}
\hline $\begin{array}{l}\text { SAVY 4000 Serial Number } \\
\text { LANMAS ID }\end{array}$ & $\begin{array}{c}\mathbf{0 3 1 1 0 5 0 5 2 ( L / B )} \\
\text { CXLOX082911 }\end{array}$ \\
\hline Material Type & 52 \\
\hline IDC & C217 \\
\hline Chemical Form & Compound Dioxide \\
\hline Material Creation Date & $2011-09-19$ \\
\hline Material Mass (g) & 786.9 \\
\hline Material Wattage (W) & 2.07 \\
\hline Initial Packaging Date & $2012-01-18$ \\
\hline
\end{tabular}

\section{Surveillance Data}

\begin{tabular}{|c|c|c|c|c|c|c|c|c|}
\hline $\begin{array}{l}\text { Surv } \\
\text { FY }\end{array}$ & $\begin{array}{l}\text { Surv. } \\
\text { Date }\end{array}$ & $\begin{array}{l}\text { SAVY } 4000 \\
\text { Age }(y)\end{array}$ & $\begin{array}{l}\text { Leak Rate } \\
\text { (atm-cc/s) }\end{array}$ & $\begin{array}{l}\text { O-ring Durometer } \\
\text { (Shore M) }\end{array}$ & $\begin{array}{c}\text { Filter Pressure } \\
\text { Drop } \\
\text { (in. W.C.) } \\
\end{array}$ & $\begin{array}{c}\text { Particle } \\
\text { Penetration (\%) }\end{array}$ & $\begin{array}{l}\text { Initial Package } \\
\text { Mass }(\mathrm{g})\end{array}$ & $\begin{array}{c}\text { Repackaged. } \\
\text { Mass (g) }\end{array}$ \\
\hline FY15 & $2015-08-26$ & 3.03 & $2.5 \times 10^{-8}$ & 57.2 & 0.563 & 0.0004 & 3136.2 & 6755.5 \\
\hline FY16 & 2016-03-07 & 4.13 & $2.6 \times 10^{-8}$ & 56.7 & 0.49 & 0.0002 & 6758.5 & 6752.3 \\
\hline FY17 & $2017-03-14$ & 5.16 & $1.3 \times 10^{-8}$ & 57.2 & 0.49 & 0.0004 & 5199.3 & $\mathrm{~N} / \mathrm{A}$ \\
\hline FY18 & 2018-3-01 & 6.1 & $3.1 \times 10^{-8}$ & 57.5 & 0.51 & 0.0001 & 6763.1 & $\mathrm{~N} / \mathrm{A}$ \\
\hline \multicolumn{9}{|c|}{ Surveillance Observations } \\
\hline $\begin{array}{l}\text { Surv } \\
\text { FY }\end{array}$ & \multicolumn{3}{|c|}{ Container Inspection } & $\begin{array}{c}\text { Corrosion } \\
\text { Ranking }\end{array}$ & \multicolumn{4}{|c|}{ Bag Inspection } \\
\hline FY15 & \multicolumn{3}{|c|}{ No comments entered } & 0 & \multicolumn{4}{|l|}{ Good } \\
\hline FY16 & \multicolumn{3}{|c|}{ Filter has no debris or discoloration } & 0 & \multicolumn{4}{|l|}{ Good } \\
\hline FY17 & \multicolumn{3}{|c|}{ No comments entered } & 0 & \multicolumn{4}{|l|}{ Good } \\
\hline FY18 & \multicolumn{3}{|c|}{ Thumb latch is a little sticky but still works } & & \multicolumn{4}{|l|}{ Good } \\
\hline
\end{tabular}


Table 23. Relevant data for annual measurements on the four NDE-only containers.

\begin{tabular}{|l|c|}
\hline $\begin{array}{l}\text { SAVY 4000 Serial Number } \\
\text { LANMAS ID }\end{array}$ & $\begin{array}{c}\text { 031105051(L/B) } \\
\text { ROTRBJ-1C1 }\end{array}$ \\
\hline Material Type & 52 \\
\hline IDC & R260 \\
\hline Chemical Form & Process Residue, Filter Residue \\
\hline Material Creation Date & $1992-11-03$ \\
\hline Material Mass (g) & 452 \\
\hline Material Wattage (W) & 1.19 \\
\hline Initial Packaging Date & $2011-08-16$ \\
\hline
\end{tabular}

\section{Surveillance Data}

\begin{tabular}{|c|c|c|c|c|c|c|c|c|}
\hline $\begin{array}{l}\text { Surv } \\
\text { FY }\end{array}$ & $\begin{array}{l}\text { Surv. } \\
\text { Date }\end{array}$ & $\begin{array}{c}\text { SAVY } 4000 \\
\text { Age (y) }\end{array}$ & $\begin{array}{l}\text { Leak Rate } \\
(\mathrm{atm}-\mathrm{cc} / \mathrm{s})\end{array}$ & $\begin{array}{l}\text { O-ring Durometer } \\
\text { (Shore M) }\end{array}$ & $\begin{array}{c}\text { Filter Pressure } \\
\text { Drop } \\
\text { (in. W.C.) }\end{array}$ & $\begin{array}{c}\text { Particle } \\
\text { Penetration (\%) }\end{array}$ & $\begin{array}{l}\text { Initial Package } \\
\text { Mass (g) }\end{array}$ & $\begin{array}{c}\text { Repackaged. } \\
\text { Mass (g) }\end{array}$ \\
\hline FY15 & $2015-01-29$ & 3.01 & $3.0 \times 10^{-8}$ & 56.5 & 0.542 & 0.0001 & 8971.7 & 8992.8 \\
\hline FY16 & 2016-03-07 & 4.11 & $2.7 \times 10^{-8}$ & 58.1 & 0.537 & 0.0000 & 8998.7 & 9193.9 \\
\hline FY17 & 2017-03-14 & 5.58 & $2.2 \times 10^{-8}$ & 58.0 & 0.54 & 0.0005 & 7616. & $\mathrm{~N} / \mathrm{A}$ \\
\hline FY18 & 2018-3-01 & 6.6 & $3.3 \times 10^{-8}$ & 57.8 & 0.56 & 0.0002 & 9208.6 & N/A \\
\hline
\end{tabular}

Surveillance Observations

\begin{tabular}{|c|l|c|l|}
\hline $\begin{array}{c}\text { Surv } \\
\text { FY }\end{array}$ & \multicolumn{1}{|c|}{ Container Inspection } & $\begin{array}{c}\text { Corrosion } \\
\text { Ranking }\end{array}$ & \multicolumn{1}{|c|}{ Bag Inspection } \\
\hline FY15 & No comments entered & 0 & Good, darkened \\
\hline FY16 & Filter has no debris or discoloration & 0 & $\begin{array}{l}\text { Brittle, not able to see inner container due to dark outer bag (added } \\
\text { additional bag) }\end{array}$ \\
\hline FY17 & $\begin{array}{l}\text { Markings of bag-out bag on interior of container, possibly the } \\
\text { beginning of corrosion. Container wiped with a slight removal of } \\
\text { the suspected corrosion. } \\
\text { Photos taken both before and after wiping }\end{array}$ & 1 & Good (overbag) \\
\hline FY18 & $\begin{array}{l}\text { Sticky thumb latch and small amounts of residue throughout the } \\
\text { inside of the container. Small dimple on bottom of the container. }\end{array}$ & & Good (overbag) \\
\hline
\end{tabular}


Table 24. Relevant data for annual measurements on the four NDE-only containers.

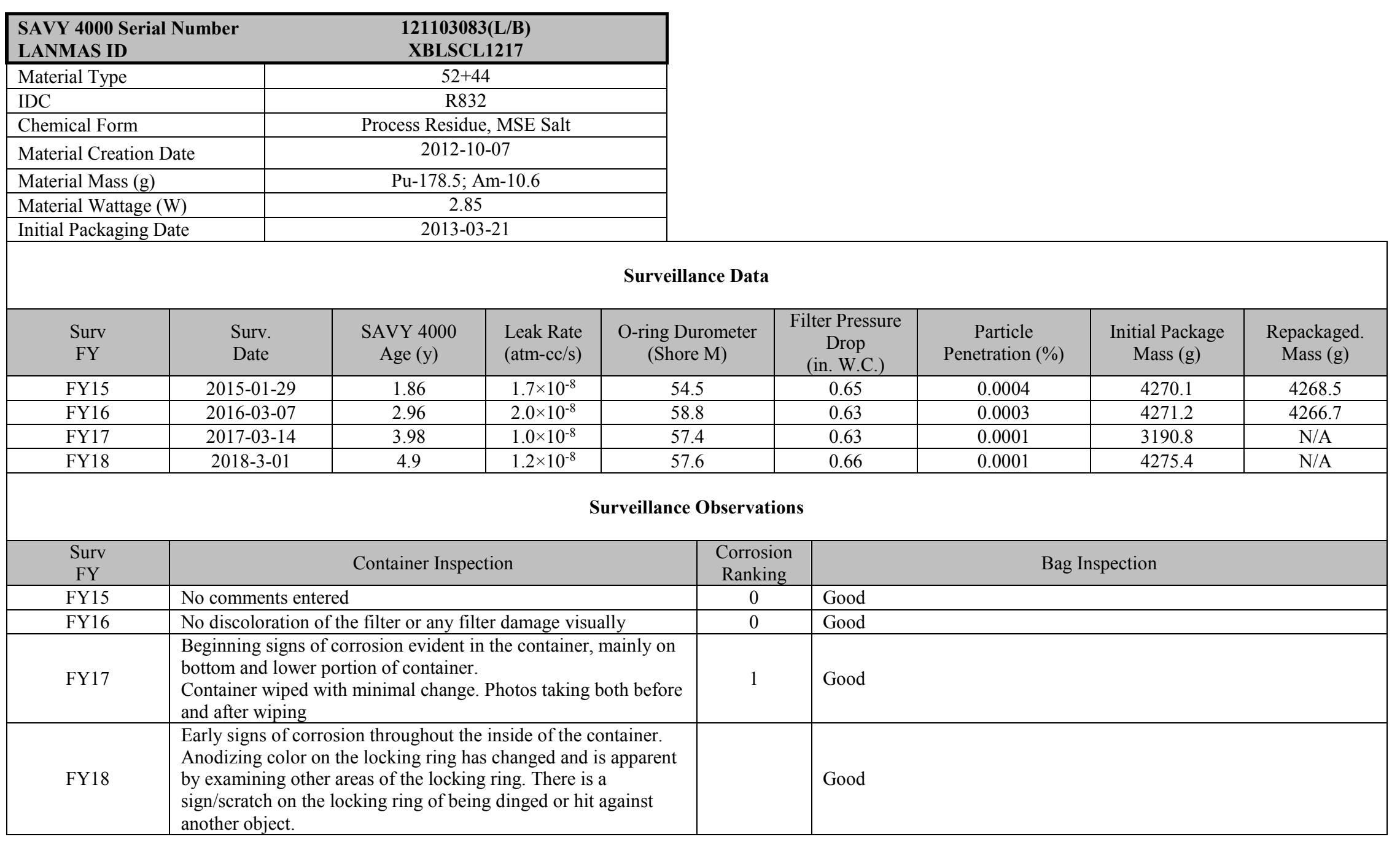


Table 25. Relevant data for annual measurements on the four NDE-only containers.

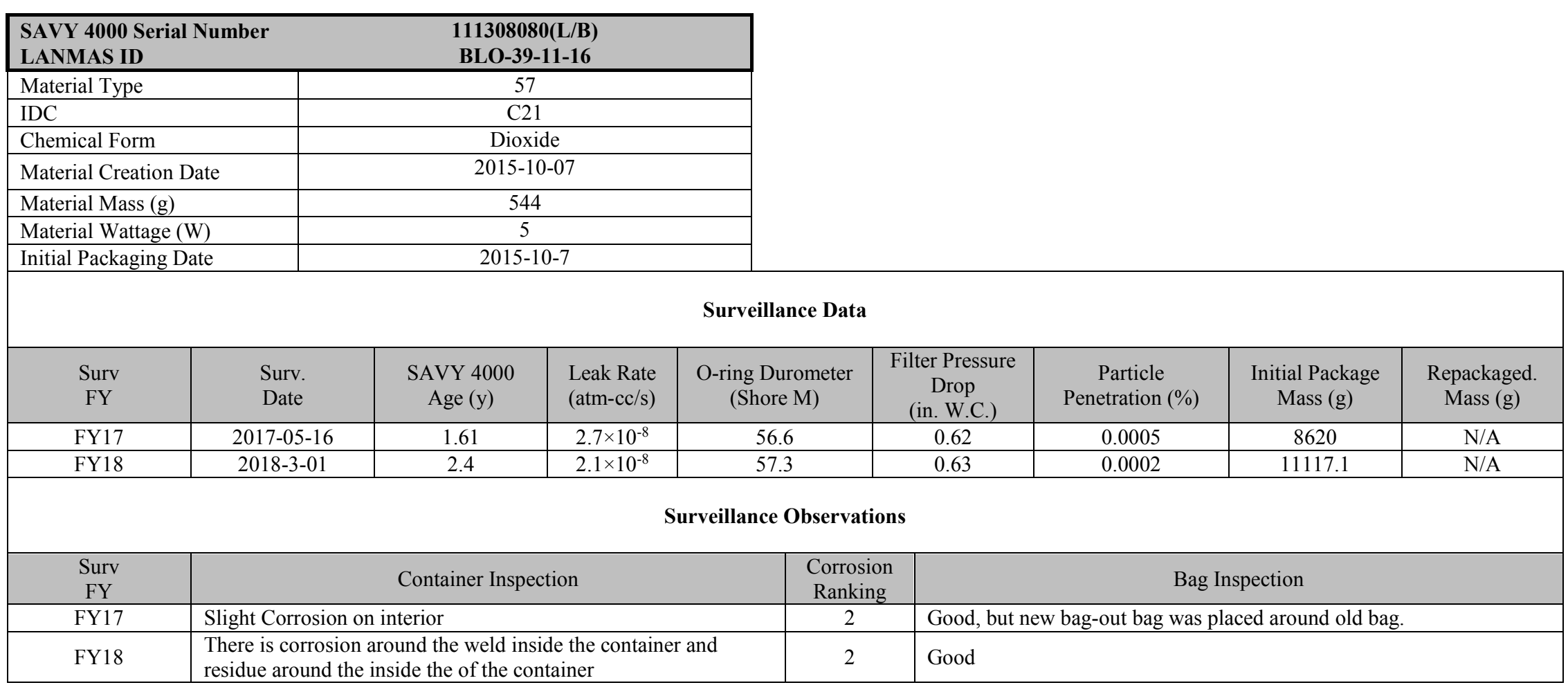




\subsection{Trending Analysis}

\subsubsection{Helium Leak Rate}

Figure 4-30 shows He leak rate versus age. The containers denoted with colored circles are the five containers that have been examined at least twice in 2015 through 2018 . The black circles are the remainder of the SAVY surveillance containers (16 containers). To date, there is no trend with time for leak rate. There is one outlier, XBLSCL1213. This container was corroded and it required cleaning to pass the leak test. This container will be examined again in 2019.

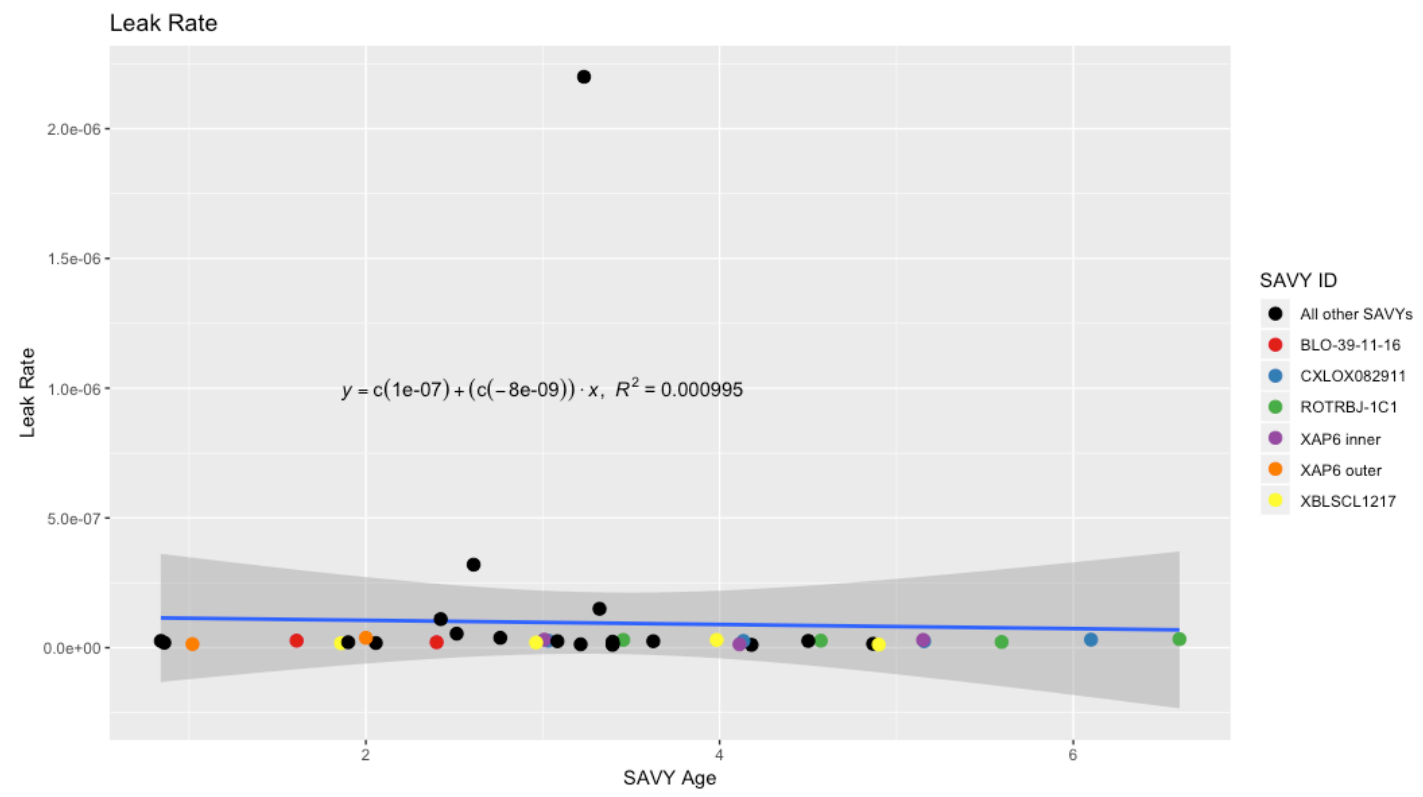

Figure 4-31. He leak rate versus SAVY age.

\subsubsection{Corrosion}

Corrosion has been found on 4 SAVY 4000 containers and 3 Hagan containers in surveillance this year. The amount of the corrosion on the SAVY 4000 containers was minor in appearance and seemed to be concentrated were it appeared the bag-out bag was touching the side of the container. The Hagan containers had more general corrosion as seen in Figure 4-4 compared to Figure 4-14. The corrosion on Hagan containers was seen beyond the O-ring seal and on the threads of the lid on container $18 \mathrm{H} 7$.

The worst appearing corrosion on SAVYs was found on container $18 \mathrm{~S} 1$. The material in this container was a plutonium oxide with $8.2 \%$ americium, packaged for one year. The worst appearing Hagan container for this year held an MSE salt with an age of 13 years.

\subsubsection{SAVY Corrosion Trending}

The corrosion ranking scale was applied to all the SAVY 4000 containers from 2015 to 2018 (Section 3). A total of 29 containers were ranked. Of these, 16 were ranked as having no corrosion and 13 had some level of corrosion. Only on SAVY container was ranked in category 3 , the highest level of corrosion. Factors that may be associated with 
corrosion include age (a), wattage (w), and container size. The bag degradation factor (BDF) [6] combines all three of these factors into a single value. BDF is defined as age times wattage divided by the square of the radius ${ }^{\circledR}$ of the container $\left(B D F=a^{*} w / r^{\wedge} 2\right)$. Figure 4-32 shows a boxplot of the BDF for each corrosion category. A boxplot (or box and whiskers plot) (McGill, R. (1978)) is a standardized way of describing the data based upon the minimum, first quartile $(25 \%)$, median, third quartile $(75 \%)$ and maximum. The rectangle spans the first and third quartile, the median segments the rectangle. The variability among the corrosion ranking categories is similar, but the median increases as the ranking increases. As the BDF takes into account wattage, age and container size it is an indication that these factors can interact to increase corrosion. The sample sizes are small, only a few observations per boxplot, so conclusions are difficult, however, as more data are collected the BDF effect will be more reliably evaluated.

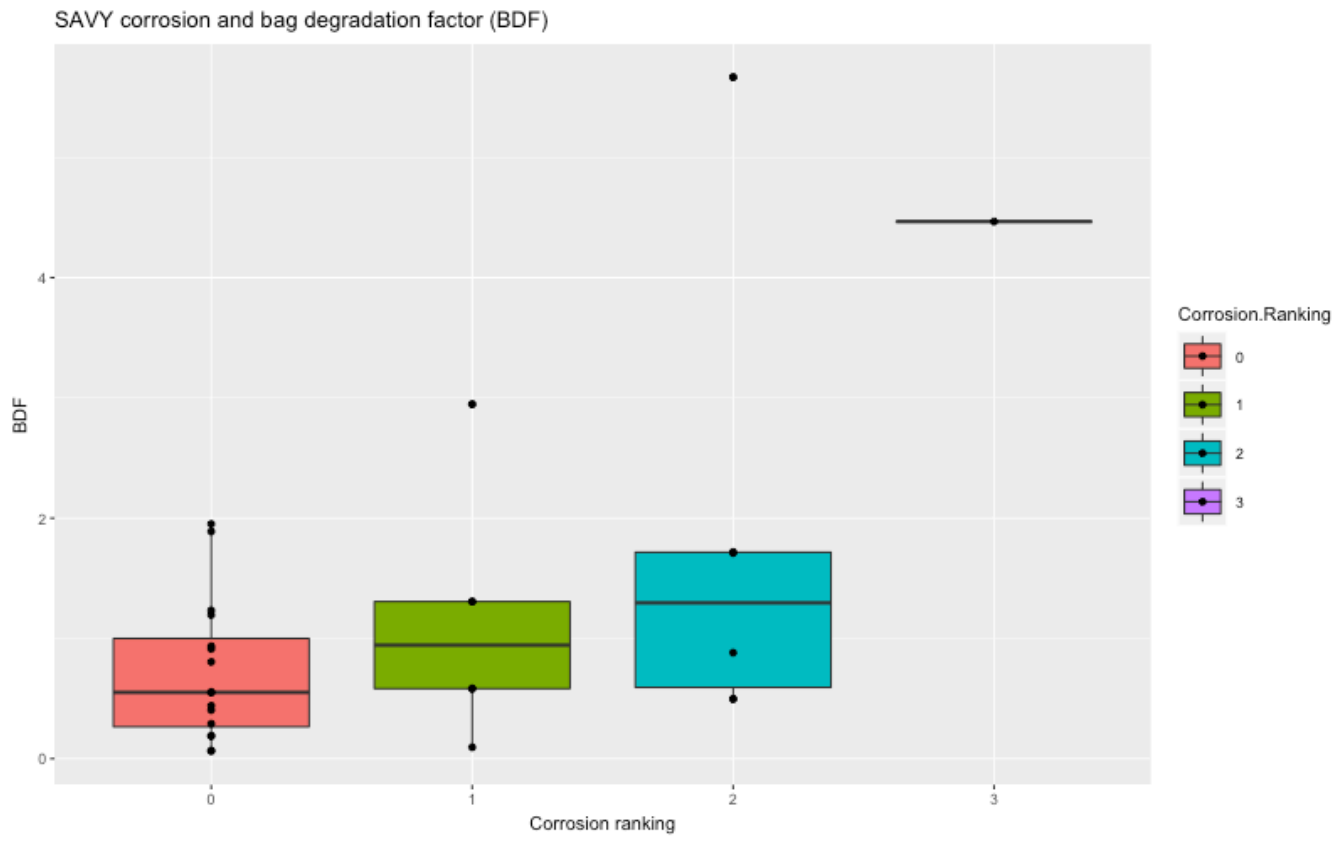

Figure 4-32. Boxplots of BDF for the various corrosion ranks for the SAVY 4000.

Figure 4-33 displays boxplots of wattage for each of the rankings for the SAVY 4000. As can be seen in the figure, the wattage values vary within the no corrosion group with three containers having a higher wattage compared to the rest. However, the higher corrosion category does have the highest wattage. 


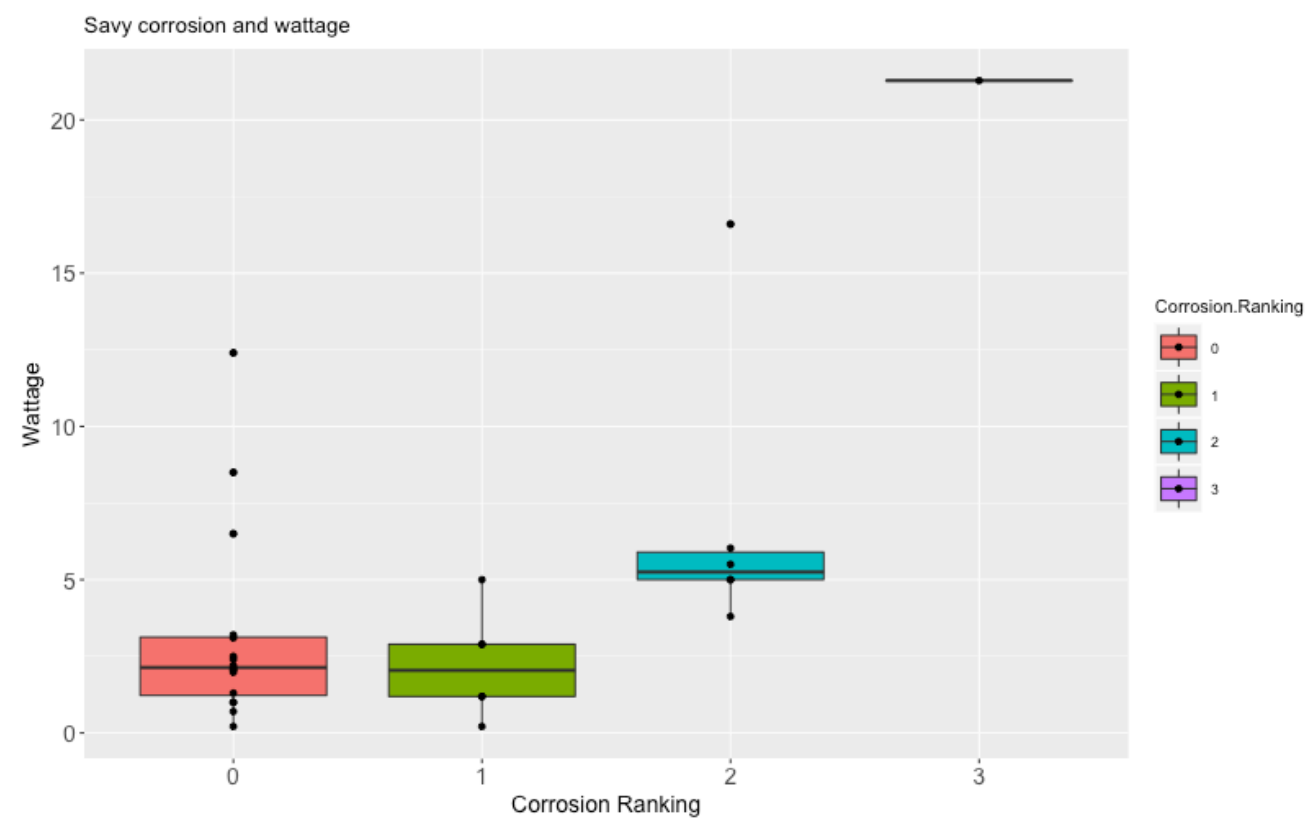

Figure 4-33. Boxplots of wattage versus corrosion rankings for the SAVY 4000.

Figure 4-34 shows a boxplots of the age versus corrosion rankings for the SAVY 4000. The age varies across all the corrosion levels suggesting that age of the SAVY 4000 alone cannot predict corrosion levels. However, the numbers of containers are small for the corrosion ranks, ranging from 1 to 5 observations. There was only one SAVY container at the highest corrosion ranking. One container that has been tracked for multiple years has changed from a state of no corrosion to a corrosion rank 1 in 2018.

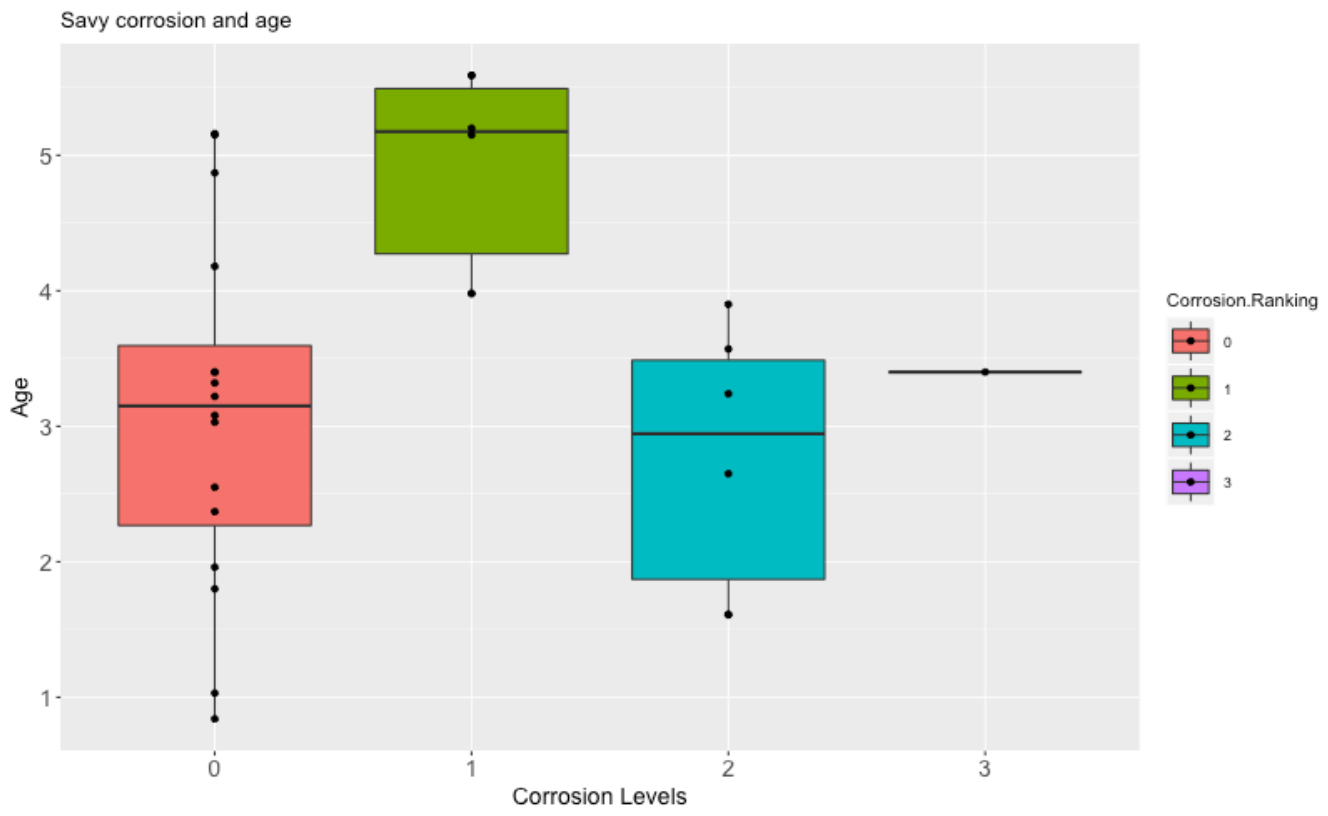

Figure 4-34. Boxplots of age versus corrosion rankings for the SAVY 4000. 


\subsubsection{Hagan Corrosion Trending}

The corrosion ranking scale was applied to all the Hagan containers from 2013 to 2018. A total of 29 containers were inspected based upon visual analysis. Of the 29 containers ranked, 10 were labeled as having no corrosion and 19 had some level of corrosion, with three Hagan containers labeled in category 3, the highest level of corrosion. Again, factors that may be associated with corrosion include age, wattage, and container size. Figure 4-35 shows a boxplot of the BDF for each corrosion category. The most variability occurs in category 1 and 3 with a spread of BDF values. However, generally there is a slight increase in the median BDF value the higher the ranking.

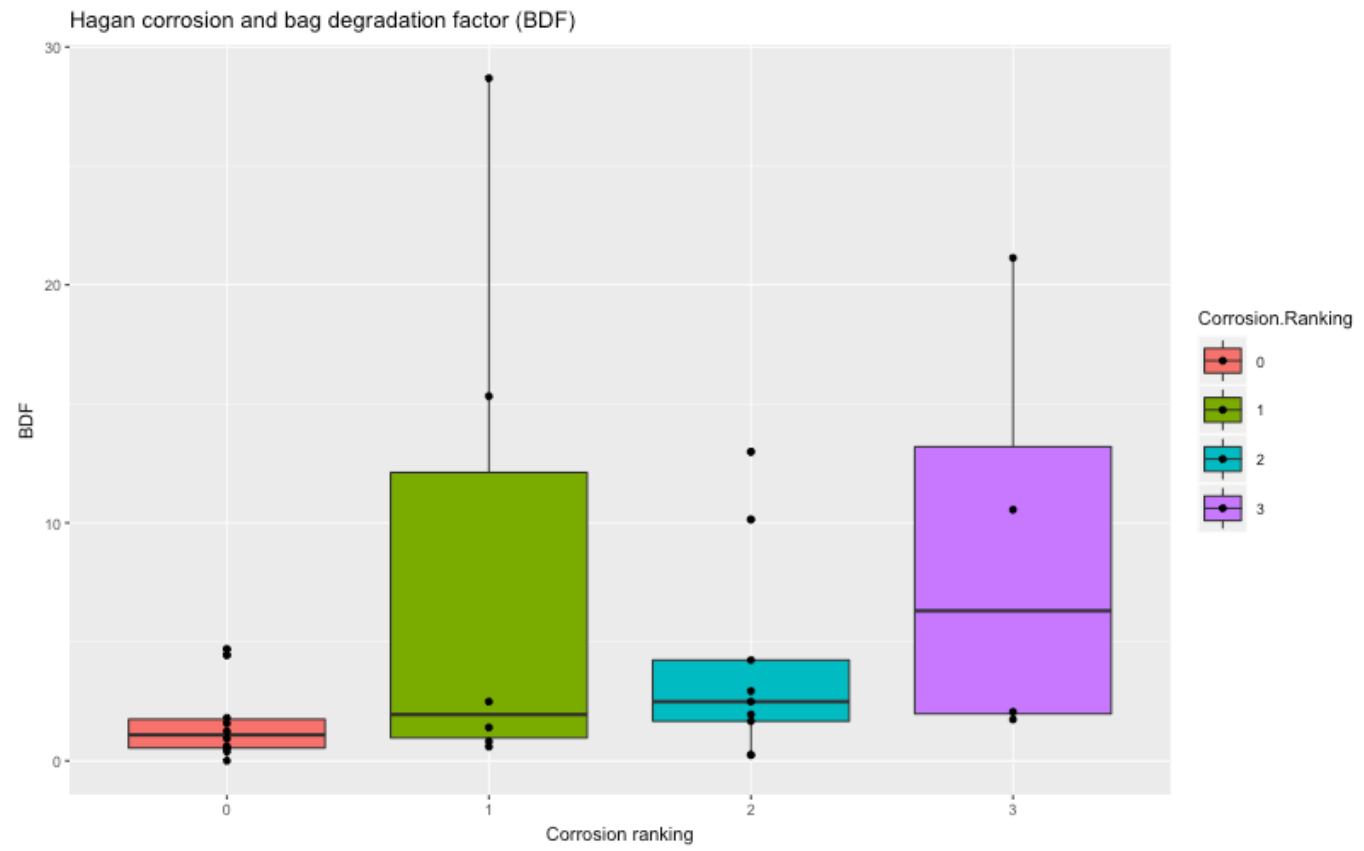

Figure 4-35. Boxplots of BDF versus corrosion rankings for Hagan containers.

Figure 4-36shows a boxplot of the age and corrosion levels. There is a lot of variability in the age especially for the non-corroded containers suggesting that age alone does not predict corrosion. 


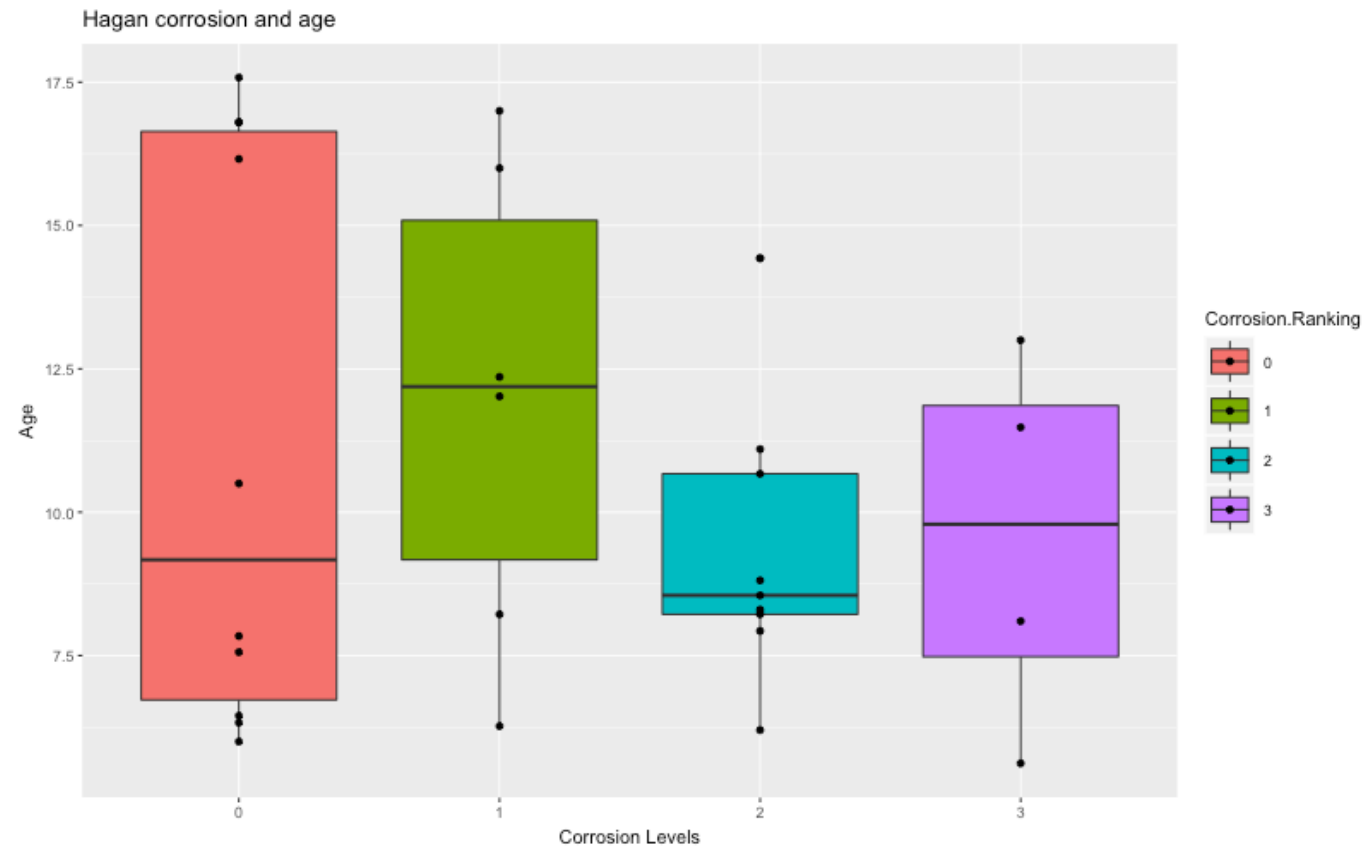

Figure 4-36. Boxplots of age versus corrosion rankings for the Hagan containers.

Figure 4-37 displays a boxplot of wattage for each of the rankings for the Hagan. In general, the wattage level is higher for the containers in categories 1,2, and 3 . The greatest amount of variability occurs in category 1 where corrosion is starting to appear.

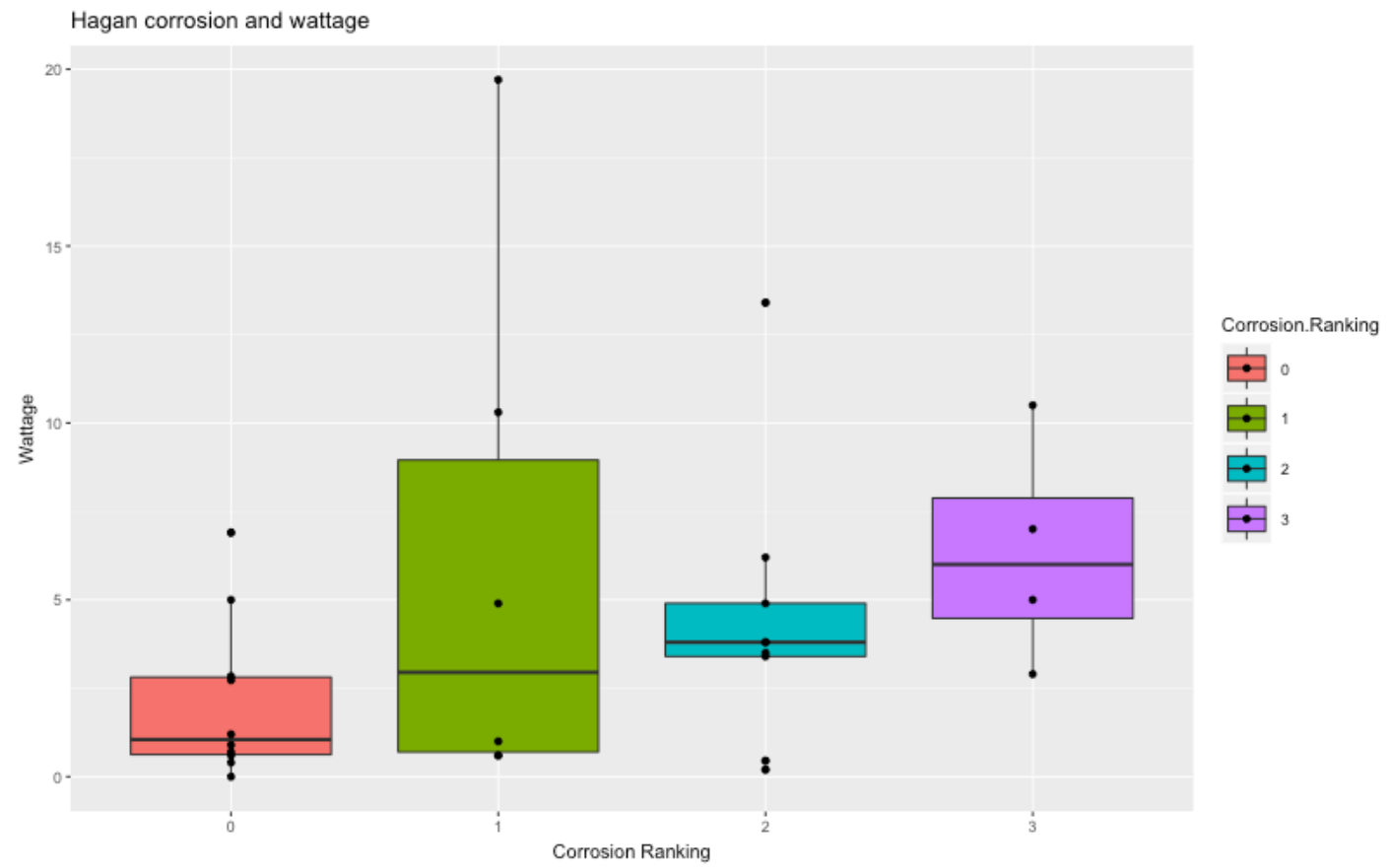

Figure 4-37. Boxplots of wattage versus corrosion rankings for the Hagan containers. 


\subsubsection{O-ring Issues}

There were no significant O-ring issues found in the SAVY 4000 containers this year. There were small inclusions or scuffmarks seen but the issues did not affect the performance on the O-ring. Figure 4-31 shows durometer measurements versus SAVY age. Again, there is considerable scatter and no significant trend.

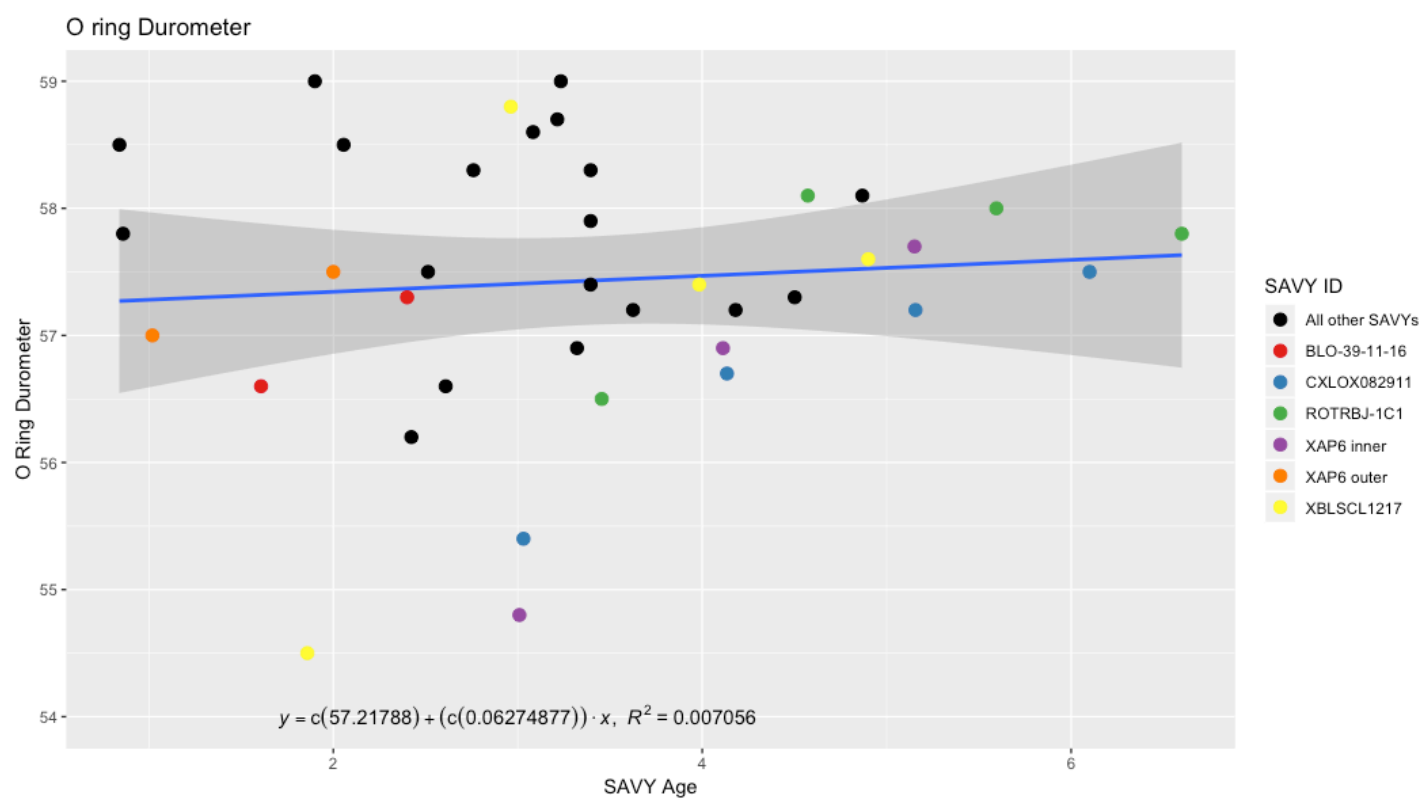

Figure 4-38. Durometer measurements versus SAVY age.

Figure 4-32 shows compression versus age. Details of how the compression data are collected and results for accelerated aging studies are provided in Weiss, et. al. 2016. The surveillance compression data were not collected in 2015 and there were only three measurements in 2017. In 2018, only three measurements were made and two were estimated based upon the difference of the manufacture measured body-sealing diameter and an average lid-sealing diameter. The average value was $6.6355 \mathrm{in}$. with a standard deviation of 0.0012 in.. The estimates for the two measurements (CLXOX08191 and ROTRBJ-1C1, blue circles) were higher than most of the other measurements, and may be artificially high because of the estimated gland size. These containers will be measured in future years and will be added to this trending analysis.

Although there is no statistically significant trend with age at this time (at the $\mathrm{p}=0.05$ level), the eye perceives an apparent increase with age. However, this apparent increase depends on the two estimated values - CLXOX08191 and ROTRBJ-1C1. As noted previously, these measurements could be artificially elevated. The three outliers, SLT1303 (green, examined in 2017), XAP6 (purple, examined in 2018), and XBLSCL1217 (green, examined in 2018) will also be tracked in future surveillances. Only one container had more than one measurement (BLO-39-11-16 red circles)[8]. 


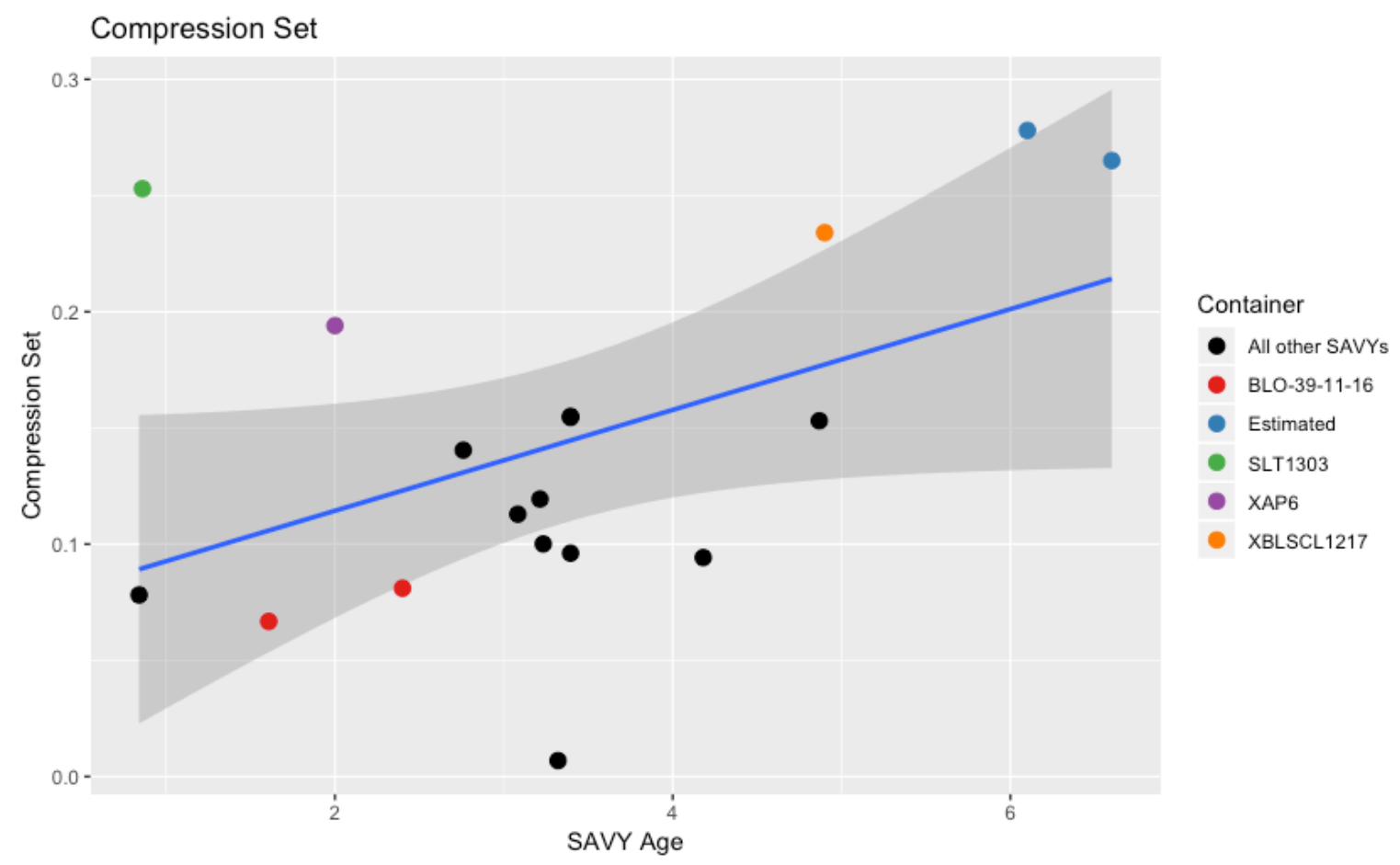

Figure 4-39. Compression set versus SAVY age.

\subsubsection{Filter Discussion}

Figure 4-33 shows particle penetration versus SAVY age. This measurement depends on how long the data are collected and this collection time varies between measurements. Variability in the collection time results in considerable variability between measurements. Therefore, particle penetration measurements will not be useful for detecting trends unless there is a very large trend over time. The data in this plot are all within the variability of the measurements. 


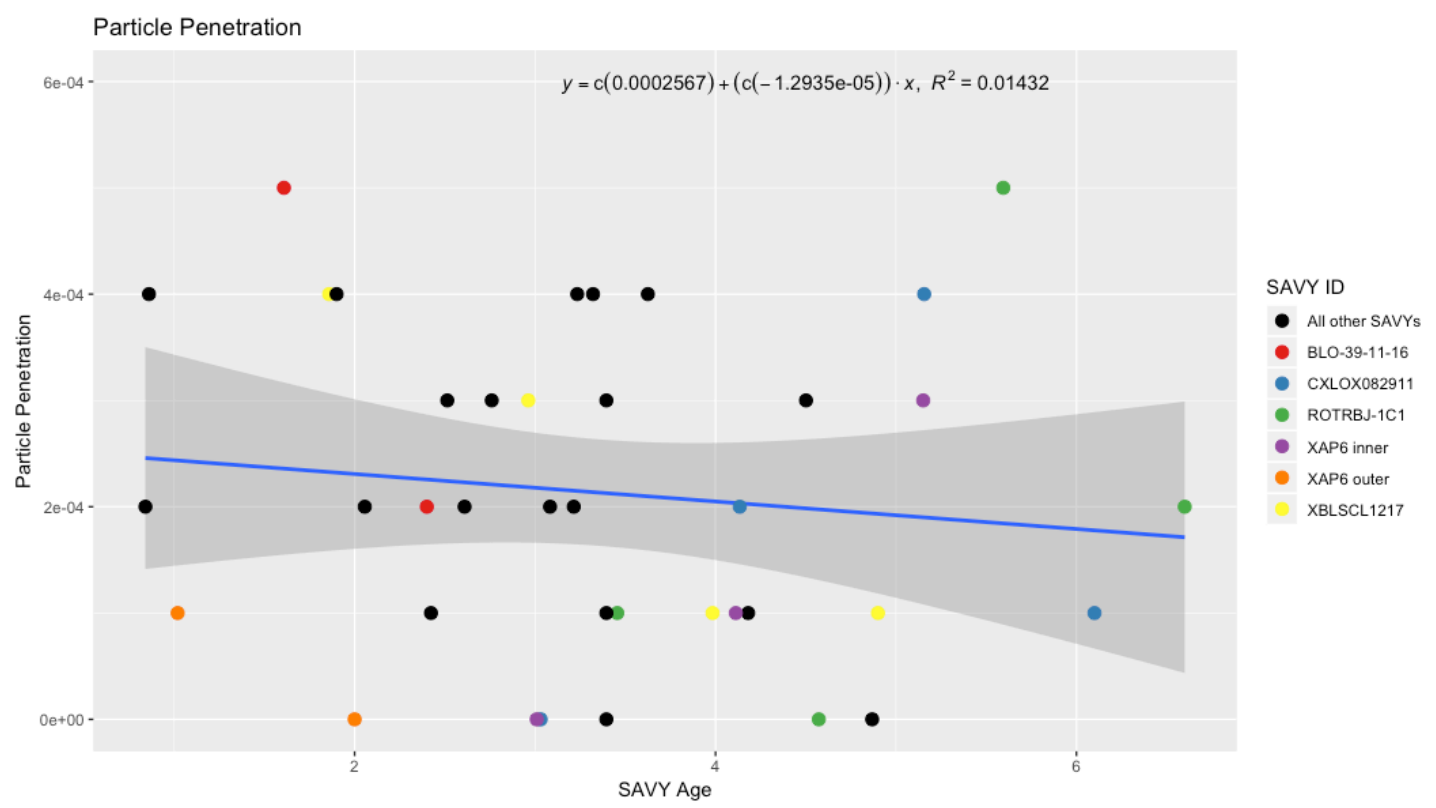

Figure 4-40. Particle penetration versus SAVY age.

Figure 4-34 shows the plot of filter pressure drop versus SAVY age. Looking at all of the data, there is a statistically significant trend $(\mathrm{p}$-value $=0.01)$, however, there is considerable scatter and the five containers that have been measured multiple times (colored circles) show no change with age. The pressure drop must be $<1$ inch water column at a flow rate of $200 \mathrm{cc} / \mathrm{min}$ to be considered passing. To date, all filters have passed.

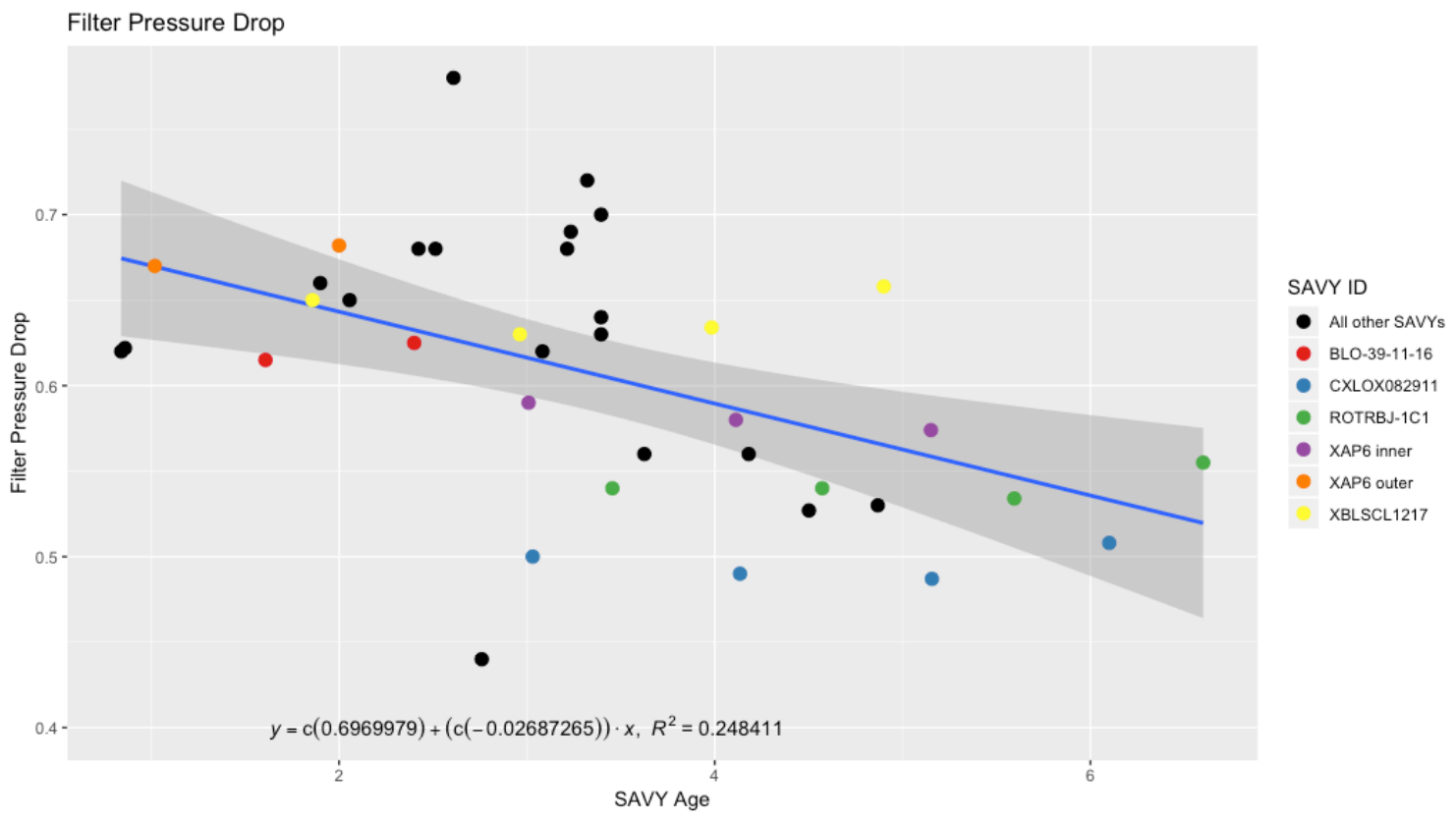

Figure 4-41. Filter pressure drop versus SAVY age. 


\section{Summary and Conclusions}

This year surveillance activities continued to find corrosion inside of the storage containers. The four SAVY 4000 containers that were found to have minor corrosion on the inner surfaces were returned to service and will be surveilled going forward. It has been noticed that there are concentrations of corrosion where we believe the bag-out bag is in contact with the walls of the container. Out of the five SAVY containers fully surveilled in FY18 there were no failure. The worst case of corrosion seen in FY18 was container $18 \mathrm{H} 7$, which clearly had corrosion beyond the sealing surface but did not have any contamination on the outside surfaces. The fact that contamination has not been found on any of the containers external surfaces indicates that the containers are performing their primary function of maintaining their contamination barrier and protecting the workers that uses these containers on a daily bases.

The most noteworthy data collected in FY18 was from 18H7. The pits observbed due to corrosion were measured and showed that the rate of growth is within the previously estimated pit growth rate outlined in the FY18 corrosion report [9]. This data on pit growth is valueable because it ferthure supports a lifetime of well greater than 15 years. Other notable observations on $18 \mathrm{H} 7$ were that no cracks from corrosion were seen.

\section{Recommendations}

\subsection{In-glovebox Capabilities}

An ability to evaluate container performance inside of gloveboxes would be a valuable improvement to the surveillance activities. Due to the number of containers being introduced into gloveboxes having the capability to measure; O-ring thickness, helium leak testing and water ingress testing, would allow for information to be gathered on containers that would otherwise be lost.

\subsection{Random Sampling}

The past surveillance plan focused on 12 IDC groups identified as worst case materials due to dose and the generation of corrosive gases that could challenge container components. Additionally, the selection prioritized the containers in these groups with the highest potential for corrosion. In FY19, it is recommended that a random sample of these groups is included in the surveillance program to ensure that the surveillance sample includes containers that are representative and bounding of the storage population.

\subsection{Material Types and Forms}

The FY19 surveillance plan should continue to focus on the 12 IDC groups identified as worst case materials. In particular, sampling should target a Hagan packaged with MSE salt similar to $18 \mathrm{H} 7$, which had a large dose in a small volume container. The condition of this container was the worst seen to date in surveillance. However, in order to ensure that the surveillance program bounds the inventory of stored material, it is recommended that the surveillance program includes all nuclear material types and material forms in the 
storage population. Materials such as neptunium materials (MT 82) and Pu-242 materials (MT 41 and 42) have not yet been sampled in surveillance and can be prioritized by factors such a dose and the potential for corrosion.

\subsection{First Alternative Bag-out Bag Surveillance Item}

In order to assess the real world performance of the alternative bag-out bag material a surveillance item that would challenge the material will need to be selected. The value of using a surveillance item is that it could serve both the container surveillance activities and help in evaluating whether the bag material should be used around the plutonium facility.

\subsection{Photo Capability for Surveillance}

It has recently been recognized that a capability to capture photos in the lab that surveiallance takes place would be greatly improve the effectiveness of evaluating the growth of corrosion in containers that are being surveilled on a regular bases. By capturing photos in the lab it would be possible to control the lighting so that the color of the photos remains consistent from year-to-year allowing a more comprehensive analysis to be completed on the photos that are captured. If a camera was available it would allow the container surveillance team to gather photos of every container that passes through the program. 


\section{References}

1. E. J. Kelly et al., "Los Alamos National Laboratory SAVY-4000 Field Surveillance Plan Update for 2018" (LA-UR-18-24442, Los Alamos, NM, 2018

2. Stone, Timothy A. et al, "SAVY-4000 Surveillance and Life Extension Program Fiscal Year 2013 Annual Report" (LA-UR-14-21362, Los Alamos, NM, 2014)

3. Reeves, Kirk et al,. "Surveillance Report of SAVY 4000 and Hagan Nuclear Material Containers for FY2016" (LA-UR-16-27427, Los Alamos, NM, 2017)

4. Reeves, Kirk et al, "Surveillance Report on SAVY-4000 and Hagan Nuclear Material Storage Containers for FY 2017" (LA-UR-17-31263, Los Alamos, 2017)

5. Stone, Timothy Amos et al., "Technical Basis Update for Design Life Extension of the SAVY-4000 Series Containers" (LA-UR-18-27269, Los Alamos, NM, 2018)

6. L.L. Anderson et al., "Safety Analysis Report for the SAVY 40004000 Container Series" (LA-CP-13-00403, Los Alamos, NM, 2013)

7. Smith, Paul Herrick et al., "Using Container Survey Data to Assign Risk to Nuclear Material Packages" (LA-UR-17-24589, Los Alamos, NM, 2017)

8. McGill, Robert, Tukey, J.W., \& Larsen, Wayne A. (February 1978). "Variations of Box Plots". The American Statistician. 32 (1): 12-16.

9. Reeves, Kirk et al., "Evaluating Corrosion Effects on the Stainless Steel Components of the SAVY-4000/Hagan Nuclear Material Storage Containers" (LA-UR-18-25709, Los Alamos, NM, 2018 